\title{
CATÁLOGO DE LOS ÁFIDOS (HEMIPTERA, APHIDIDAE) DE CHILE, CON PLANTAS HOSPEDADORAS Y DISTRIBUCIONES REGIONAL Y PROVINCIAL
}

\author{
Juan M. Nieto Nafría ${ }^{1}$, Eduardo Fuentes-Contreras ${ }^{2}$, Marta Castro Colmenero ${ }^{3}$, Marta Aldea Piera ${ }^{4}$, \\ Jaime Ortego ${ }^{5}$ \& M. Pilar Mier Durante ${ }^{6}$
}

\author{
1Departamento de Biodiversidad y Gestión Ambiental, área de Zoología. Universidad de León. 24197 León (España); jmnien@unileon.es \\ ${ }^{2}$ Universidad de Talca. Centro de Ecología Molecular y Aplicaciones evolutivas en agroecosistemas - Núcleo Milenio (CEM). \\ Facultad de Ciencias Agrarias. Casilla 747. Talca (Chile); efuentes@utalca.cl \\ ${ }^{3}$ Departamento de Biodiversidad y Gestión Ambiental, área de Zoología. Universidad de León. 24197 León (España); \\ mcastc@estudiantes.unileon.es \\ ${ }^{4}$ Departamento de Biodiversidad y Gestión Ambiental, área de Zoología. Universidad de León. 24197 León (España); \\ maldep@estudiantes.unileon.es
}

5INTA EEA Mendoza. San Martín, 3853. 5507 Luján de Cuyo (Mendoza, Argentina); ortego.jaime@inta.gob.ar

${ }^{6}$ Departamento de Biodiversidad y Gestión Ambiental, área de Zoología. Universidad de León. 24197 León (España); mpmied@unileon.es

\section{RESUMEN}

Se han revisado críticamente las citas en Chile de especies y subespecies de áfidos (Aphididae) contenidas en 139 contribuciones científicas. Se añaden citas de numerosas especies a partir de especímenes de las colecciones del Muséum national d'Histoire naturelle (París, Francia), del Servicio Agrícola y Ganadero (SAG) (Santiago de Chile, Chile), del Natural History Museum (Londres, Reino Unido), de las universidades de León (España) y Talca (Chile) y de J. Ortego (Mendoza, Argentina). El catálogo afídico chileno queda formado por 169 especies y subespecies, quedando excluidas de él Aphis (Toxoptera) citricidus, Aphis medicaginis, Aphis rumicis, Dysaphis apiifolia petroselini, Lipaphis erysimi y Uroleucon aaroni. Se citan por primera vez en América del Sur Aphis rubicola, Brachycaudus amygdalinus e Illinoia pepperi. Se citan por primera vez en Chile Aphis forbesi, Aphis senecionicoides, Hyadaphis passerini, Hyperomyzus carduellinus, Illinoia azaleae, Neotoxoptera oliveri y Pentalonia nigronervosa. Se dan más de un centenar de primeras citas regionales o provinciales de especies. Se presenta por primera vez la relación de plantas hospedadoras de los pulgones citados en el país. Se comentan las composiciones de las afidofaunas regionales y provinciales. Se ofrece una valoración de los componentes alóctono y autóctono de la afidofauna chilena.

Palabras clave: Áfidos; pulgones; Aphididae; Chile; catálogo; plantas hospedadoras; distribución.

\section{ABSTRACT}

Catalogue of aphid species (Hemiptera, Aphididae) of Chile, with host plants and regional and provincial distributions

Records of the aphid species and subspecies contained in 139 scientific contributions have been revised. New records of many species are presented from specimens conserved in the collections of Muséum national d'Histoire naturelle (Paris, France), the Servicio Agrícola y Ganadero (SAG) (Santiago, Chile), the Natural History Museum (London, U.K.), the universities of León (Spain) and Talca (Chile) and of J. Ortego (Mendoza, Argentina). The Chilean aphid-catalog is composed of 169 species and subspecies; Aphis (Toxoptera) citricidus, Aphis medicaginis, Aphis rumicis, Dysaphis apiifolia petroselini, Lipaphis erysimi and Uroleucon aaroni are excluded from the catalog. Aphis rubicola, Brachycaudus amygdalinus and Illinoia pepperi are first recorded from South America. Aphis forbesi, Aphis senecionicoides, Hyadaphis passerini, Hyperomyzus carduellinus, Illinoia azaleae, Neotoxoptera oliveri and Pentalonia nigronervosa are also recorded for first time from Chile. More a hundred new regional and provincial records are given. Host plant list of aphids recorded in Chile are presented for first time. The compositions of Chilean regional and provincial aphidfaunas are discussed. An evaluation of the allochthonous and autochthonous components of the Chilean aphidfauna is presented.

Key words: Aphids; Aphididae; Chile; catalogue; host plants; distribution. 


\begin{abstract}
Cómo citar este artículo/Citation: Nieto Nafría, J. M., Fuentes-Contreras, E., Castro Colmenero, M., Aldea Piera, M., Ortego, J. \& Mier Durante, M. P. 2016. Catálogo de los áfidos (Hemiptera, Aphididae) de Chile, con plantas hospedadoras y distribuciones regional y provincial. Grael/sia, 72(2): e050. http://dx.doi.org/10.3989/graellsia.2016.v72.167
\end{abstract}

Copyright: (C) 2016 SAM y CSIC. Salvo indicación contraria, todos los contenidos de la edición electrónica de Graellsia se distribuyen bajo licencia de uso y distribución Creative Commons Reconocimiento no Comercial 3.0. España (cc-by-nc).

\section{Introducción}

Los datos faunísticos aparecen no solamente en las publicaciones de temáticas faunística (estudios de taxofaunas de territorios de extensión variada) o taxonómica (establecimiento de nuevas especies o descripciones o redescripciones de formas) sino también en publicaciones de entomología aplicada, de agronomía (incluyendo los avisos de especies de interés agrícola), de ecología o de estudios filogenéticos. Esta dispersión es un obstáculo evidente para el buen conocimiento de las faunas cualquiera que sea su ámbito geográfico de referencia; obstáculo que solamente puede salvarse con trabajos de recensión o con catálogos, como los de Smith \& Cermeli (1979), referido a América al sur de la frontera estadounidense mexicana, o los de Fuentes-Contreras et al. (1997) y Anónimo (2010), referidos a Chile, y como el que aquí se presenta, también referido a Chile.

La primera referencia de un áfido en Chile se debe a Le Feuvre (1875), quién informó de la presencia en el país de Schizoneura lanigera (actualmente Eriosoma lanigerum (Hausmann), el pulgón lanígero del manzano) desde aproximadamente 1850. Una década después, Philippi (1885) hace un recuento de las especies conocidas en Chile en ese momento que se consideraban introducidas y menciona a Aphis rosae Linnaeus (actualmente Macrosiphum rosae) y de nuevo al pulgón lanígero del manzano. Años después, van der Goot (1912) publicó la descripción de una nueva especie, Aphis citricola (actualmente sinónima de $A$. fabae Scopoli), a partir de ejemplares recogidos sobre un cítrico indeterminado en una localidad chilena sin precisar. La siguiente contribución, de Porter (1916), es muy peculiar, pues informa de la depredación de Leptasthenura aegithaloides (Kittlitz, 1830) (Aves, Passeriformes, Furnariidae) sobre pulgones, cuya identificación no proporciona el autor y que no es posible establecer ahora, quizás alguna especie de Macrosiphum o Aulacorthum. Las más recientes aportaciones al conocimiento de la fauna de pulgones de Chile son las de Nieto Nafría et al. (2015) sobre la ausencia de Aphis (Toxoptera) citricidus (Kirkaldy) en Chile y de López Ciruelos et al. (2016) con la descripción de Aphis maulensis Mier Durante \& García-Tejero.

\section{Material y métodos}

\section{ORgANIZACIÓN DEL CATÁLOGO}

Se toma la familia Aphididae Latreille, 1802 con la extensión taxonómica fijada en la "clasificación de Remaudière, Stroyan y Quednau ampliada"
(Nieto Nafría \& Favret, 2011), y quedan por lo tanto filoxéridos y adélgidos fuera del catálogo. Para las subespecies se ha seguido el criterio de Blackman \& Eastop (2016). Los nombres válidos de las especies y de las subespecies se han ajustado al catálogo en línea Aphid Species File (Favret, 2014) y las referencias de autores y publicaciones de los géneros y subgéneros pueden hallarse en Nieto Nafría et al. (2011).

\section{REGISTROS BIBLIOGRÁFICOS}

Para establecer los registros de las especies de pulgones (Aphididae) en Chile se han examinado más de 150 publicaciones (en papel o en la red) y se ha extraído información de las 139 siguientes (2 del siglo XIX, 9 de la primera mitad del siglo XX, 82 de su segunda mitad y 46 de estos primeros años del siglo XXI): Le Feuvre (1875), Philippi (1885), van der Goot (1912), Porter (1916, 1917, 1922, 1923), Mújica (1941), Capdeville (1945), Olalquiaga Fauré (1944, 1946), Campos (1953), Essig (1953), Caltagirone (1957), Commonwealth Institute of Entomology (1964a, 1964b), Rojas (1966), Zúñiga (1967a, 1967b, 1967c, 1968), Commonwealth Institute of Entomology (1968), Hille Ris Lambers (1968), Richards (1968), Commonwealth Institute of Entomology (1969, 1971a, 1971b, 1971c), Lara \& Zúñiga (1969), Apablaza (1973), Apablaza \& Tiska (1973), Campos \& Peña(1973), Carrillo (1974), Carrillo \& Zúñiga (1974), Carrillo et al. (1974), Carrillo \& Mellado (1975), Commonwealth Insitute of Entomology (1975), Hille Ris Lambers (1975), Starý (1976), Zúñiga \& Suzuki (1976), Carrillo (1977), Baltra (1979), Smith \& Cermeli (1979), Commonwealth Institute of Entomology (1979), Carrillo (1980), Cortázar Sagarminaga (1980), Herrera \& Quiroz (1980), Quiroz (1980), Suzuki (1981), Vargas (1981), Vargas \& Suzuki (1984), Commonwealth Institute of Entomology (1986), CAB International Institute of Entomology (1986), Quiroz et al. (1986), Zerené et al. (1988), CAB International Institute of Entomology (1989), Gerding \& Figueroa (1989), Gerding et al. (1989), Zúñiga \& Aguilera (1989), Quednau (1990), Charlín (1991), International Institute of Entomology (1991), Gerding \& Norambuena (1991), Henríquez (1991), Norambuena \& Gerding (1991), Prado (1991), Remaudière et al. (1993), Starý (1993), Aguilera \& Ortega (1994), Aguilera et al. (1994), Blackman \& Eastop (1994), Quednau \& Remaudière (1994), Remaudière (1994), Starý (1994), Starý et al. (1994a, 1994b), Aguilera \& Pacheco (1995), Carrillo \& Mundaca (1995), Muñoz (1995), Muñoz \& Beèche (1995), Starý (1995), Heie et al. (1996), Eastop et al. (1997), Fuentes-Contreras et al. (1997), Ortego \& 
Mier Durante (1997), Ortego (1998), de Carvalho et al. (1998), Gonzales et al. (1998a, 1998b), Nieto Nafría et al. (1999), Quiroz et al. (1999), Caballero et al. (2000), Klein Koch \& Waterhouse (2000), Niemeyer et al. (2002), Ramírez (2002), Blackman et al. (2003), Mier Durante et al. (2003), Beèche et al. (2004), Fuentes-Contreras et al. (2004), Gaete et al. (2004), Ortego et al. (2004), Basoalto et al. (2005), Delfino \& Gonzales (2005), Servicio Agrícola \& Ganadero (2005a, 2005b, 2005c), Aguilera et al. (2006a, 2006b), Blackman \& Eastop (2006), Quirós \& Emmen (2006), Servicio Agrícola \& Ganadero (2006a, 2006b), Fuentes-Contreras et al. (2007), Nieto Nafría et al. (2007), Ortego et al. (2007), Forestry Department FAO (2008), Nieto Nafría et al. (2008), Peccoud et al. (2008), Ripa et al. (2008a, 2008b, 2008c), Lavandero et al. (2009), Anónimo (2010), Cabrera-Brandt et al. (2010), Montalva et al. (2010), Servicio Agrícola \& Ganadero (2010), Ripa et al. (2010), Castañeda et al. (2011), González (2011), Lavandero et al. (2011), Lupichini \& Ripa (2011), Rubio-Meléndez et al. (2011), Lavandero et al. (2012), Ortego et al. (2013), Astorga (2014), Machuca (2014), Starý et al. (2014), Nieto Nafría et al. (2015) y López Ciruelos et al. (2016).

Cada una de las citas contenidas en esas contribuciones dio lugar a un registro, entendiendo por tal la relación entre una especie (o subespecie) de pulgón con una planta (o en su lugar con otra forma de captura) y con una localización: localidad concreta, provincia, región o la nación en general.

La información contenida en cada uno de los registros se valoró teniendo en cuenta: 1) las características morfológicas o biológicas del pulgón correspondiente suministradas por el autor; 2) la bibliografía citada en la contribución; 3) la historia de las interpretaciones taxonómicas y la de las citas en Chile; y 4) la distribución y la bionomía del pulgón actualmente admitidas, teniendo muy en cuenta lo expuesto por Blackman \& Eastop (2016).

Los nombres de los géneros y de las especies de algunas de las plantas mencionadas se han ajustado a usos taxonómicos actuales teniendo en cuenta lo expuesto por Tropicos.org (2016) y por Blackman \& Eastop (2016).

Las regiones y en su caso las provincias se han fijado a partir: 1) de la localidad de captura que en cada caso se mencionara; 2 ) en ausencia de localidad concreta a partir de la provincia mencionada, teniendo en cuenta el año de la publicación, pues ha habido modificaciones en los nombres y límites provinciales; o 3) en ausencia de provincia a partir de la región mencionada desde 1975, año en el que se establecieron éstas. Si la cita se dio en una serie de regiones en bloque, por ejemplo: «de la IV a la X» (Prado, 1991), se ha considerado que la especie está citada en cada una de las regiones incluidas en el bloque. Las citas genéricas a las regiones I o X han dado lugar a registros en estas regiones, aún a riesgo de que en realidad pudieran corresponder respectivamente también o en exclusiva a las regiones XV o XIV.

\section{Nuevas CitAS}

Para formar el catálogo también se han tenido en cuenta datos sin publicar del material de las colecciones del Muséum national d'Historie naturelle (París, Francia), del Servicio Agrícola y Ganadero (Santiago de Chile, Chile), de la Universidad de León (León, España), del Natural History Museum (Londres, Reino Unido), de Jaime Ortego (Mendoza, Argentina), y que aportasen novedades en cuanto a plantas hospedadoras o provincias con relación a las citas bibliográficas.

La mayor parte de las muestras de la colección del Museo de París fueron recogidas en los años 1991 y 1992 por P. Starý. Otras son de los años 1966, 1967, 1974, 1995, 2000, y sus recolectores son varios. En todo caso las identificaciones son de G. Remaudière.

Las muestras conservadas en la colección del Servicio Agrícola y Ganadero (SAG) son de procedencias y fechas muy variadas.

Las muestras chilenas de la colección de la Universidad de León fueron recogidas por $\mathrm{T}$. Cekálovic Kuschevich (Universidad de Concepción, Chile) en los períodos 1957-1963 y 1976-1998, por uno de los autores (O.) en el período 2000-2004 o por tres de los autores (N.N., M.D. y O.) en 2000. Las identificaciones son de alguno de esos tres autores.

De la colección del Natural History Museum se han tenido en cuenta muestras de varias especies colectadas por G. Kushel, G. Monsalve y E. Zúñiga entre 1958 y 1974 y por D. Hille Ris Lambers en 1974 e identificadas por este último.

Las muestras chilenas de la colección de Jaime Ortego fueron recogidas e identificadas por él mismo.

\section{Resultados y discusión}

\section{El catálogo chileno de Áfidos (Hem. Aphididae)}

Este catálogo incluye 169 taxones del grupo especie, computando como un solo taxón especie y subespecie nominotípica, y no computando la especie si en Chile no se ha citado la nominotípica correspondiente.

El orden de presentación es alfabético, sin considerar el nombre del subgénero, que se aporta cuando no es el nominotípico. En la Tabla 2 se consigna la adscripción taxonómica supragenérica de acuerdo con la "clasificación de Remaudière, Stroyan y Quednau ampliada" (Nieto Nafría \& Favret, 2011).

Para cada especie o subespecie se dan por separado las plantas sobre las que se han citado o se citan ("Sobre") y las regiones y en su caso provincias en donde se han citado o se citan ("En"). Las plantas se relacionan alfabéticamente; el simple nombre de un género o de una familia significa que el autor de la cita no precisó una identificación botánica más concreta. Los nombres genéricos de las plantas se abrevian cuando ya han sido mencionados con una a cinco letras según corresponda para evitar equívocos en cada una de las relaciones. Las Regiones se relacionan ordenadas de 
Norte a Sur, utilizando su ordinal y «M» para la Región Metropolitana; en concreto XV (de Arica y Parinacota), I (de Tarapacá), II (de Antofagasta), III (de Atacama), IV (de Coquimbo), V (de Valparaíso), M (Metropolitana de Santiago), VI (del Libertador General Bernardo O’Higgins), VII (del Maule), VIII (del Bío Bío), IX (de La Araucanía), XIV (de Los Ríos), X (de Los Lagos), XI (de Aisén del General Carlos Ibáñez del Campo), XII (de Magallanes y de la Antártica Chilena). Las provincias se relacionan alfabéticamente dentro del paréntesis que sigue al número de la Región correspondiente. Ambas listas, la de plantas hospedadoras y la de regiones y provincias son independientes entre sí y no se debe pensar que un pulgón sea conocido sobre todas las plantas mencionadas en cada una de las regiones y provincias en las que haya sido citado.

Las nuevas citas pulgón-planta, pulgón-región y pulgón-provincia, que se aportan en este estudio se señalan con una ene mayúscula entre paréntesis y como superíndice, $\left\langle\left({ }^{(\mathrm{N})}\right\rangle\right.$, por detrás del nombre de la planta, de la región o de la provincia, cuyos datos concretos no se aportan por brevedad dado su elevado número.

En algunos casos se han añadido comentarios que aclaran la situación en Chile del pulgón correspondiente, incluyendo los registros que se consideran accidentales en razón de sus plantas hospedadoras. En el caso de las especies que se citan por primera vez en Chile, se informa de la presencia de la especie concernida solamente en Argentina, Bolivia y Perú, países limítrofes con Chile.

Acyrthosiphon kondoi Shinji, 1938.- Sobre Caesalpinia angulata, Lens culinaris, Lupinus, Medicago sativa, Melilotus, Mel. albus, Mel. indica, Pisum sativum y Trifolium. La cita de esta especie (Starý, 1994) sobre Canna se puede considerar accidental. En las Regiones I (El Tamarugal ${ }^{(\mathbb{N})}$, Iquique $\left.{ }^{(\mathrm{N})}\right)$, II (El Loa $^{(\mathrm{N})}$ ), III (Huasco), IV (Elqui), V (Quillota, San Antonio, San Felipe de Aconcagua, Valparaíso), M, VI, VII (Talca), VIII (Ñuble), IX (Cautín, Malleco), XIV, X y XI.

Acyrthosiphon malvae malvae (Mosley, 1841). - En las citas bibliográficas no consta la subespecie, posiblemente la nominotípica, como en la nueva cita. Sobre Geranium, Pelargonium ${ }^{(\mathrm{N})}$ y Malva. En las Regiones $I^{(\mathrm{N})}$ (Iquique $\left.^{(\mathrm{N})}\right)$, $\mathrm{I}^{(\mathrm{N})}$ (Copiapó $\left.^{(\mathrm{N})}\right)$, V (Quillota, San Felipe de Aconcagua ${ }^{(\mathrm{N})}$, Valparaíso) y VIII (Ñuble).

Acyrthosiphon pisum (Harris, 1776).- Sobre Astragalus germaini, Glycine max, Lathyrus latifolius, La. subandinus, Lens culinaris, Lotus, Lupinus, Medicago, M. arabica, M. sativa, Melilotus indica, Pisum sativum, Trifolium pratense, T. repens, Vicia benghalensis, V. faba, V. sativa y V. villosa. En las Regiones I, II, III, IV, V (Los Andes, Petorca, Quillota, San Antonio, San Felipe de Aconcagua, Valparaíso), M (Cordillera ${ }^{(\mathrm{N})}$, Melipilla, Santiago), VI $\left(\right.$ Cachapoal $\left.^{(\mathrm{N})}\right)$, VII (Cauquenes, Linares, Talca), VIII (Arauco, Bío Bío ${ }^{(\mathrm{N})}$, Concepción ${ }^{(\mathrm{N})}$, Nuble), IX (Cautín, Malleco), XIV (Ranco $^{(\mathrm{N})}$, Valdivia), X (Llanquihue ${ }^{(\mathrm{N})}$, Osorno $\left.^{(\mathrm{N})}\right)$ y XI, XII ${ }^{(\mathrm{N})}$ (Magallanes $^{(\mathrm{N})}$, Última Esperanza ${ }^{(\mathrm{N})}$ ).

Aphis alstroemeriae Essig, 1953.- Sobre Alstroemeria. En la Región V (Los Andes).

Aphis asclepiadis Fitch, 1851.- Especie mencionada siempre en la bibliografía chilena con el nombre de $A$. helianthi Monell, 1879, su sinónimo posterior, como también lo es $A$. carduella Walsh, 1863, nombre usado para ella hasta hace poco tiempo
(Lagos-Kutz et al., 2016). Sobre Helianthus annuus. En las Regiones XV (Arica) y I.

Aphis (Toxoptera) aurantii Boyer de Fonscolombe, 1841.Toxoptera debe ser considerado un subgénero de Aphis Linnaeus de acuerdo con lo expuesto por Lagos et al. (2014). Sobre Citrus, C. limon, C. maxima, C. reticulata, Eucalyptus ${ }^{(\mathbb{N})}$ y Persea americana. En las Regiones XV, I, II (Antofagasta ${ }^{(\mathrm{N})}$ ), III, IV, V (Isla de Pascua, Petorca, Quillota, Valparaíso), M, VI, VII y VIII (Bío Bío( ${ }^{(\mathrm{N})}$, Concepción $\left.{ }^{\mathrm{N})}\right)$.

Aphis berberidorum Ortego \& Mier Durante, 1997.- Sobre Berberis darwinii. En la Región XI (General Carrera).

Aphis carrilloi Ortego, Mier Durante \& Nieto Nafría, 2013.Sobre Gunnera magellanica. En la Región VII (Talca).

Aphis conflicta Nieto Nafría, Ortego \& Mier Durante, 2008.Sobre Colletia, C. spinosissima, Discaria chacaye, Trevoa quinquenervia y Tristerix. En las Regiones M (Cordillera), VII (Talca), VIII (Concepción, Ñuble), IX (Malleco).

Aphis craccivora craccivora Koch, 1854.- La mayor parte de las citas chilenas de Aphis craccivora deben referirse a la subespecie nominotípica, aunque los autores correspondientes no lo expresaran explícitamente; las nuevas citas lo son con certeza. A esta subespecie deben atribuirse las citas de A. medicaginis Koch, 1854 dadas por Essig (1953) — quien la consideraba sinónima anterior de A. craccivora-; A. medicaginis es monoica holocíclica sobre especies de Medicago, mientras que $A$. craccivora tiene un espectro alimenticio mucho más amplio, además $A$. medicaginis - de distribución europea—no se ha citado nunca en América del Sur. Sobre Acacia $^{(\mathbb{N})}$ Adesmia $^{(\mathbb{N})}$, Artemisia, Asteraceae, Baccharis, Baccharis salicifolia ${ }^{(\mathrm{N})}$, Brassica oleracea var. capitata, Br. oleracea var. viridis, Cactaceae, Cassia, Cereus, Chusquea, Citrullus lanatus, Citrus maxima, Citrus x sinensis $^{(\mathrm{N})}$, Cucurbita ficifolia, Cu. maxima, Cynara cardunculus, Fabaceae, Geoffroea decorticans ${ }^{(\mathrm{N})}$, Helianthus annuus, Lens culinaris, Lotus, Malus domestica, Medicago, Med. sativa, Melilotus indica, Mutisia subulata ${ }^{(\mathrm{N})}$, Nasturtium officinale, Nicotiana tabacum, Phaseolus vulgaris, Pisum sativum, Prosopis tamarugo, Pyrus communis, Quillaja saponaria ${ }^{(\mathbb{N})}$, Robinia pseudoacacia, Rubus idaeus, Simmondsia chinensis, Solanum, So. nigrum, So. tuberosum, Trifolium, T. pratense, T. repens, Vicia faba y V. sativa. En las Regiones XV (Arica), I, II, III (Copiapó( ${ }^{(\mathrm{N})}$ ), IV (Elqui), V (Isla de Pascua, Los Andes, Marga Marga, Quillota, San Felipe de Aconcagua, Valparaíso), M (Melipilla ${ }^{(\mathrm{N})}$, Santiago), VI $\left(\right.$ Cachapoal $\left.^{(\mathrm{N})}\right)$, VII $\left(\right.$ Talca $\left.^{(\mathrm{N})}\right)$, VIII (Bío Bío, Ñuble), IX y X.

Aphis craccivora pseudacaciae Takahashi, 1966.- Esta subespecie fue citada explícitamente por Carrillo (1980), aunque se pueden albergar dudas de su presencia en Chile, ya que según Blackman \& Eastop (2016) la subespecie está restringida al Extremo Oriente asiático, y todo el material de Aphis craccivora recogido sobre la misma planta, Robinia pseudoacacia, en Argentina pertenece a la subespecie nominotípica. La presencia de la subespecie debería confirmarse estudiando de nuevo el material que dio origen a la cita o por nuevas colectas. Sobre Robinia pseudoacacia. En Región M (Santiago).

Aphis danielae Remaudière, 1994.- Sobre Echinopsis chiloensis y Lycium stenophyllum. En la Región IV (Elqui).

Aphis fabae fabae Scopoli, 1763.- La complejidad morfológica de esta especie y la dificultad para separar sus subespecies e incluso para separarla de especies muy próximas no permite asegurar cuál de esas subespecies podría haber sido citada en cada caso, si bien lo más probable es que se trate de la subespecie nominotípica. A esta especie deben atribuirse las citas de $A$. rumicis De Geer, 1773 dadas por Essig (1953), quien la consideraba sinónima - y lógicamente sinónima anteriorde $A$. fabae, porque $A$. rumicis es monoica holocíclica sobre 
especies de Rumex, mientras que $A$. fabae es básicamente dioica y en el hospedador secundario polífaga y frecuentemente paracíclica holocíclica; $A$. rumicis no se ha citado nunca con certidumbre en América del Sur. La mención hecha por Ortego et al. (2013) de A. fabae eryngii Blanchard (E.E.), 1923 - conocida sólo en Argentina - debe tenerse por una errata. Sobre Beta vulgaris, B. vulgaris subsp. cicla, Carpobrotus chilensis, Cestrum, Ce. parqui $^{(\mathrm{N})}$, Chrysanthemum, Ch. coronarium, Cirsium, Citrus, Cucurbita ficifolia, Cynara cardunculus, Epiphyllum, Foeniculum vulgare, Gladiolus, Helianthus annuus, Ligustrum, Medicago sativa, Nerium oleander, Phaseolus vulgaris, Pittosporum tobira, Ribes alpestre, Rumex, Simmondsia chinensis ${ }^{(\mathrm{N})}$ y Vicia faba. En las Regiones XV (Arica), I (Iquique $^{(\mathrm{N})}$ ), II (Antofagasta), III $\left(\right.$ Huasco $\left.^{(\mathrm{N})}\right)$, IV (Elqui), V (Los Andes, Marga Marga, Quillota, San Felipe de Aconcagua, Valparaíso), M (Santiago, Cordillera ${ }^{(\mathrm{N})}$ ), VI $\left(\right.$ Cachapoal $\left.^{(\mathrm{N})}\right)$, VII, VIII (Arauco, Bío Bío ${ }^{(\mathrm{N})}$, Ñuble) IX (Malleco) y X (Osorno).

Aphis forbesi Weed, 1889.- Primera cita en Chile de esta especie neártica a partir de especímenes conservados en la colección del SAG. De origen norteamericano, es conocida en América del Sur desde hace tiempo (Smith \& Cermeli, 1979). Sobre Fragaria x ananassa. En las Regiones I (El Tamarugal), VI (Ñuble) y VIII (Bío Bío).

Aphis gossypii Glover, 1877.- Sobre Allium cepa, Annona cherimola, Asparagus officinalis, Bignonia, Brassica oleracea, Br. oleracea var. botrytis, $\mathrm{Br}$. oleracea var. capitata, $\mathrm{Br}$. oleracea var. viridis, Capsella bursa-pastoris, Citrullus lanatus, Citrus, Citrus limon, Citrus maxima, Cucumis melo, Cucurbita ficifolia, Cucur. maxima, Cynara cardunculus, Daucus carota, Eriobotrya japonica, Fragaria, Gerbera, Glycine max, Gossypium, Go. hirsutum, Helianthus annuus, Jacaranda, Lamiaceae ${ }^{(\mathrm{N})}$, Malus domestica, Medicago sativa, Paulownia, Persea americana, Phaseolus vulgaris, Pisum sativum, Pitraea cuneato-ovata ${ }^{(\mathbb{N})}$, Psidium guajava, Punica granatum, Pyrus communis, Solanum lycopersicon, S. tuberosum, Trifolium, T. pratense, T. repens, Vaccinium y $V$. corymbosum. En las Regiones XV (Arica), I (El Tamarugal $^{(\mathrm{N})}$, Iquique $\left.{ }^{(\mathrm{N})}\right)$, II (Antofagasta), III, IV (Limarí, Elqui), V (Isla de Pascua, Quillota, San Felipe de Aconcagua, ${\text { Valparaíso), } \mathrm{M}^{(\mathrm{N})}\left(\text { Santiago }^{(\mathrm{N})}\right), \text { VI, VII }}^{(\mathrm{N})}\left(\right.$ Talca $\left.^{(\mathrm{N})}\right)$, VIII (Nuble), XIV, IX y X.

Aphis hederae Kaltenbach, 1843.- Citada con ese nombre y también (Carrillo, 1977) como A. hederae pseudohederae Theobald, 1927, que es una variante poblacional (Blackman \& Eastop, 2016). Sobre Aralia y Hedera helix. En las Regiones M (Santiago) y XIV (Valdivia).

Aphis illinoisensis Shimer, 1866.- Sobre Vitis vinifera. La cita de esta especie sobre Berberis dada por Essig (1953) debe considerarse accidental. En las Regiones III, IV, V, M, VI y X (Llanquihue).

Aphis marthae Essig, 1953.- Sobre Quillaja saponaria. En las Regiones V (San Felipe de Aconcagua), M (Cordillera, Santiago), VII ${ }^{(\mathrm{N})}\left(\right.$ Talca $\left.^{(\mathrm{N})}\right)$ y VIII (Arauco, Ñuble).

Aphis maulensis Mier Durante \& García Tejero, 2016.- Sobre Euphorbia sp., quizás E. klotzschii o E. collina. En la Región VII (Talca).

Aphis nasturtii Kaltenbach, 1843.- Sobre Drimys winteri. En la Región M (Santiago).

Aphis nerii Boyer de Fonscolombe, 1841.- Sobre Hoya carno$s a^{(\mathrm{N})}$, Nerium oleander y Tweedia birostrata. En las Regiones II (Antofagasta), V (San Felipe de Aconcagua, Quillota), M (Santiago $^{(\mathrm{N})}$, Cordillera), VI ${ }^{(\mathrm{N})}\left(\right.$ Cachapoal $\left.^{(\mathrm{N})}\right)$, VII (Talca) y X (Llanquihue).

Aphis papillosa Mier Durante, Nieto Nafría \& Ortego, 2003.Sobre Senecio filaginoides. En la Región XI (General Carrera).
Aphis patagonica Blanchard (E.E.), 1944.- Sobre Berberis microphylla. En las Regiones VII (Linares), VIII (Arauco ${ }^{(\mathrm{N})}$, Bío Bío, Nuble), IX ${ }^{(\mathrm{N})}$ (Cautín $^{(\mathrm{N})}$, Malleco $\left.^{(\mathrm{N})}\right), \mathrm{X}$ (Chiloé, Llanquihue), XI (Coihaique) y XII (Tierra del Fuego ${ }^{(\mathrm{N})}$, Última Esperanza).

Aphis roberti Nieto Nafría, Ortego \& Mier Durante, 1999.Sobre Mulinum spinosum. En la Región XI (General Carrera).

Aphis rubicola Oestlund, 1887.- Primera cita en Chile de esta especie neártica, a partir de especímenes conservados en la colección del SAG. Sobre Rubus idaeus. En la Región VIII (Bío Bío). Primera cita de la especie en América del Sur.

Aphis ruborum (Börner, 1931).- Sobre Rubus y R. ulmifolius. En las Regiones V (Quillota), VI ${ }^{(\mathbb{N})}$ (Cachapoal $\left.^{(\mathrm{N})}\right)$, VII (Talca) y VIII (Arauco, Ñuble).

Aphis sambuci Linnaeus, 1758.- Sobre Sambucus y S. nigra. En las Regiones XIV (Valdivia) y XI ${ }^{(\mathrm{N})}\left(\right.$ General Carrera $\left.^{(\mathrm{N})}\right)$.

Aphis schinifoliae Blanchard (E.E.), 1939.- Sobre Schinus y S. $m$ olle ${ }^{(\mathrm{N})}$. En las Regiones III ${ }^{(\mathrm{N})}\left(\mathrm{El} \mathrm{Loa}^{(\mathrm{N})}\right), \mathrm{M}^{(\mathrm{N})}\left(\right.$ Cordillera $^{(\mathrm{N})}$, Santiago $\left.{ }^{(\mathrm{N})}\right)$ y VII (Talca).

Aphis schinivora Ortego, Nieto Nafría \& Mier Durante, 2007.Sobre Schinus johnstoni. En la Región VII (Talca).

Aphis senecionicoides Blanchard (E.E.), 1944.- Primera cita en Chile: Senecio filaginoides, Chile Chico, provincia del General Carrera, Región XI de Aysén del General Carlos Ibáñez del Campo, 17 de enero de 1996, J. Ortego leg. y col. La especie es conocida en Argentina.

Aphis spiraecola Patch, 1914.- Durante los años entre 1976 y 1988 esta especie fue citada frecuentemente en todo el Mundo, y también en Chile, bajo el nombre de Aphis citricola van der Goot, por un concepto erróneo de esta especie nominal. La mención de Aphis pomi De Geer, 1773 que hizo Capdeville (1945) se debió basar en una identificación incorrecta de esta especie, a la que se parece mucho. Sobre Acacia $^{(\mathrm{N})}$, Actinidia chinensis var. deliciosa, Annona cherimola, Bahia ambrosioides, Bidens aurea, Caesalpinia angulata, Calandrinia parviflora, Citrus, Ci. aurantifolia, Ci. limon, Ci. maxima, Ci. reticulata, Cotoneaster ${ }^{(\mathrm{N})}$, Crataegus, Cydonia oblonga, Eriobotrya japonica, Jasminum ${ }^{(\mathbb{N})}$, Macadamia, Magnolia grandiflora ${ }^{(\mathbb{N})}$, Mangifera indica, Malus, Mal. domestica, Maytenus boaria, Nerium oleander ${ }^{(\mathrm{N})}$, Oenothera picensis, Pluchea absinthioides $^{(\mathrm{N})}$, Podocarpus ${ }^{(\mathrm{N})}$, Prunus, Pr. domestica, Pr. salicina, Punica granatum $^{(\mathrm{N})}$, Pyrus communis, Raphanus raphanistrum $^{(\mathrm{N})}$, Rhododendron ${ }^{(\mathrm{N})}$, Rubus idaeus, Spiraea cantoniensis $^{(\mathrm{N})}$, Viburnum tinus, Vitis vinifera y Weigela ${ }^{(\mathrm{N})}$. En las Regiones XV (Arica $\left.{ }^{(\mathrm{N})}\right)$, I (El Tamarugal), II, III (Huasco), IV (Elqui), V (Isla de Pascua, Quillota, Valparaíso $^{(\mathrm{N})}$ ), M (Santiago), VI, VII Curicó $^{(\mathrm{N})}$, Linares ${ }^{(\mathrm{N})}$, Talca $\left.{ }^{(\mathrm{N})}\right)$, VIII (Ñuble), IX $\left(\right.$ Malleco $\left.^{(\mathrm{N})}\right)$, XIV y X.

Aploneura lentisci (Passerini, 1863).- Sobre Chusquea ${ }^{(\mathrm{N})}$, Lolium y Triticum $^{(\mathrm{N})}$. En las Regiones VI (Cachapoal), VII ${ }^{(\mathrm{N})}$ (Linares $\left.^{(\mathrm{N})}\right)$, VIII $^{(\mathrm{N})}\left(\tilde{N}_{u b l e}{ }^{(\mathrm{N})}\right)$

Appendiseta robiniae (Gillette, 1907).- Sobre Robinia pseudoacacia. En las Regiones VI ${ }^{(\mathrm{N})}\left(\right.$ Cachapoal $\left.^{(\mathrm{N})}\right)$, VII (Curicó, Talca $\left.^{(\mathrm{N})}\right)$.

Aulacorthum solani solani (Kaltenbach, 1843).- Citada en Chile simplemente a nivel de especie; la distribución de las subespecies nos lleva a pensar que la citas dadas en Chile son de la subespecie nominotípica, como lo son con certeza las nuevas citas que se aportan. Sobre Apiaceae, Asparagus, Calceolaria $^{(\mathrm{N})}$, Chrysanthemum ${ }^{(\mathrm{N})}$, Citrus limon, Ci. reticulata, Ci. maxima, Cucurbita ficifolia, Cu. maxima, Cynara cardunculus, Geranium, Lactuca sativa, Medicago sativa, Phaseolus vulgaris, Pisum sativum, Poaceae, Punica granatum $^{(\mathrm{N})}$, Ruta bracteosa, Senecio ${ }^{(\mathbb{N})}$, Solanum lycopersicon, So. tuberosum, Trifolium, T. pratense y T. repens. En las Regiones V (Marga 
Marga, Quillota), M (Cordillera ${ }^{(\mathrm{N})}$, Santiago), VI $\left(\right.$ Cachapoal $\left.^{(\mathrm{N})}\right)$, VII, VIII (Bío Bío ${ }^{(\mathbb{N})}$, Concepción ${ }^{(\mathbb{N})}$, Nuble $\left.^{(\mathrm{N})}\right)$, IX $\left(\right.$ Cautín $\left.^{(\mathrm{N})}\right), \mathrm{X}$ (Chiloé(N), Llanquihue $^{(\mathrm{N})}$, Osorno) y XII ${ }^{(\mathrm{N})}\left(\right.$ Magallanes $\left.^{(\mathrm{N})}\right)$.

Brachycaudus (Thuleaphis) amygdalinus (Schouteden, 1905).Primera cita en Chile de esta especie paleártica a partir de especímenes conservados en la colección del SAG. Sobre Polygonaceae. En la Región III (Huasco). Ésta es también su primera cita en América del Sur.

Brachycaudus helichrysi (Kaltenbach, 1843).- Sobre Amsinckia hispida, Amygdalus communis, Baccharis linearis $^{(\mathrm{N})}$, Chrysanthemum, Ch. morifolium, Conyza $a^{(\mathrm{N})}$, Gardenia ${ }^{(\mathrm{N})}$, Helianthus annuus, Helminthotheca echioides, Lupinus, Lycopersium esculentum, Matricaria, Medicago sativa, Petunia $^{(\mathrm{N})}$, Prunus, Pr. divaricata ${ }^{(\mathrm{N})}$, Pr.domestica, Pr.persica, Pr. salicina, Punica granatum, Senecio, Trifolium pratense, $T$. repens y Vicia faba ${ }^{(\mathrm{N})}$. En la colección del SAG hay ejemplares de esta especie recogidos sobre Aralia que posiblemente fueran individuos errantes. En las Regiones XV (Arica, Parinacota ${ }^{(\mathrm{N})}$ ), III, IV (Elqui), V (Marga Marga, Quillota), M (Cordillera( ${ }^{(\mathrm{N})}$, Santiago), VI $\left(\right.$ Cachapoal $\left.^{(\mathrm{N})}\right)$, VII $\left(\right.$ Talca $\left.^{(\mathrm{N})}\right)$, VIII (Bío Bío( ${ }^{(\mathrm{N})}$, Nuble), IX (Cautín), XIV (Valdivia), X (Llanquihue, Palena $\left.^{(\mathrm{N})}\right), \mathrm{XI}^{(\mathrm{N})}\left(\right.$ Coihaique $\left.^{(\mathrm{N})}\right)$ y XII ${ }^{(\mathrm{N})}$ (Magallanes $^{(\mathrm{N})}$, Última Esperanza $\left.^{(\mathrm{N})}\right)$.

Brachycaudus (Scrophulaphis) persicae (Passerini, 1860).Sobre Amygdalus communis, Prunus armeniaca, P. domestica, P. persica y P. salicina. Las citas de este especie dadas sobre Lupinus por Prado (1991), sobre Solanum lycopersicon por Zúñiga (1967b) y Prado (op. cit.), sobre Malus y Malus domestica por Starý (1995), sobre Phaseolus vulgaris por Zúñiga (1967b) y sobre Pistacia vera por Prado (op. cit.) deben ser consideradas accidentales. En las Regiones I, II, III, IV, V (Quillota, Valparaíso), M (Maipo $\left.^{(\mathrm{N})}\right)$, VI $\left(\right.$ Cachapoal $\left.^{(\mathrm{N})}\right)$, VII, VIII, IX (Cautín ${ }^{(\mathrm{N})}$ ) y X.

Brachycaudus (Thuleaphis) rumexicolens (Patch, 1917).- Sobre Rumex. En las Regiones $\mathrm{V}^{(\mathrm{N})}\left(\right.$ Quillota $\left.^{(\mathrm{N})}\right)$, VIII (Arauco) y XI (General Carrera).

Brachycaudus (Appelia) schwartzi (Börner 1931).- En algunos casos esta especie ha sido citada con este nombre, y en otros (Zúñiga, 1967a; Prado, 1991; Klein Koch \& Waterhouse, 2000) bajo el de B. (A.) tragopogonis o de Anuraphis tragopogonis y viviendo en especies de Prunus. Ambas especies fueron consideradas sinónimas, son difíciles de separar morfológicamente y se diferencian bien biológicamente, pues B. schwartzi está restringida a Prunus persica y raramente a otras especies del género (Blackman \& Eastop, 2016) mientras que $B$. tragopongonis lo está a plantas del género Tragopogon. Sobre Prunus, Prunus divaricata ${ }^{(\mathbb{N})}$ y P. persica. Las citas de esta especie o de B. tragopogonis sobre Amygdalus communis, $P$. armeniaca, $P$. domestica y $P$. salicina, por Zúñiga (1967b), Prado (1991), Starý (1994) y Klein Koch \& Waterhouse (2000), se han debido fundar en identificaciones incorrectas de alguna otra especie de Brachycaudus. En las Regiones II ${ }^{(\mathrm{N})}\left(\right.$ Huasco $\left.^{(\mathrm{N})}\right)$ III $\left(\right.$ Copiapó $\left.^{(\mathrm{N})}\right)$, IV (Elqui $\left.^{(\mathrm{N})}\right)$, V (Quillota), M (Melipilla ${ }^{(\mathrm{N})}$, Santiago), VI (Cachapoal $^{(\mathrm{N})}$ ), VII, VIII y IX (Cautín, Malleco $\left.{ }^{(\mathrm{N})}\right)$. La presencia de esta especie en algunas de las regiones en las que no hay mención de provincia podría ser dudosa, porque no es posible discernir la relación entre plantas hospedadoras y regiones en citas de esa especie.

Brachycaudus (Appelia) tragopogonis (Börner, 1931).- Sin planta hospedadora. En Chile según datos de Blackman \& Eastop (2016), por lo que podría sobrentenderse que hay material chileno de esta especie en la colección del Natural History Museum de Londres. Las citas de B. tragopogonis en Chile sobre frutales no pueden tenerse en cuenta para considerar que esta especie esté presente en Chile (ver Br. schwartzi). Las citas de Zúñiga (1967b), Prado (1991) y Klein Koch \& Waterhouse (2000) de Br. tragopogonis sobre Helianthus annuus son muy dudosas; todas ellas debieron basarse en identificaciones incorrectas de otras especies de Brachycaudus, por ejemplo B. car$d u i$, la cual puede desarrollarse sobre Heliantus annuus y ya está citada en Argentina.

Brevicoryne brassicae (Linnaeus, 1758).- Sobre Beta vulgaris, Brassica, Br. napus, Br. nigra, Br. oleracea, Br. oleracea var. botrytis, $B r$. oleracea var. capitata, $B r$. oleracea var. gemmifera, Br. oleracea var. italica, Br. oleracea var. viridis, $B r$. rapa, Hirschfeldia incana, Raphanus raphanistrum, $R$. sativus, Sinapis, S. arvensis. Las citas de esta especie dadas sobre Cucumis sativus por Zúñiga (1967b), sobre Euphorbia peplus por Starý (1993, 1995), y sobre Nicotiana tabacum, Phaseolus vulgaris, Pisum sativum, Solanum lycopersicon, S. tuberosum y Trifolium por Zúñiga (1967b) deben ser consideradas accidentales. En las Regiones I, II, III, IV (Elqui), V (Isla de Pascua, Los Andes, Marga Marga, Quillota, San Felipe de Aconcagua, Valparaíso), M (Chacabuco ${ }^{(\mathrm{N})}$, Cordillera $^{(\mathrm{N})}$, Maipo $\left.^{(\mathrm{N})}\right)$, VI, VII (Curicó $^{(\mathrm{N})}$, Talca $\left.^{(\mathrm{N})}\right)$, VIII (Arauco, Nuble), IX (Cautín), XIV (Valdivia), X (Osorno), XI y XII.

Capitophorus elaeagni (Del Guercio, 1894).- Sobre Cynara cardunculus. Las citas de este especie dadas sobre Cucurbita ficifolia y Solanum lycopersicon por Prado (1991), por Zúñiga (1967b) y por Klein Koch \& Waterhouse (2000), sobre Malva por Starý (1995), y sobre Phaseolus vulgaris por Zúñiga (1967b) deberían considerarse accidentales. En las Regiones

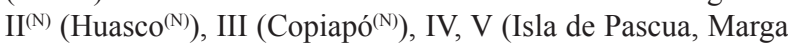
Marga, Quillota, San Felipe de Aconcagua $\left.{ }^{(\mathrm{N})}\right), \mathrm{M}\left(\right.$ Santiago $\left.^{(\mathrm{N})}\right)$, VI (Cachapoal $\left.{ }^{(\mathrm{N})}\right)$, VII, VIII (Ñuble), IX y X.

Capitophorus hippophaes javanicus Hille Ris Lambers, 1953.Es ésta la única subespecie presente en América del Sur según Blackman \& Eastop (2016); en Chile se ha citado simplemente a nivel de especie. Sobre Persicaria maculosa. En la Región VII (Talca).

Cavariella aegopodii (Scopoli, 1763).- Sobre Anethum graveolens, Apiaceae, Apium australe var. angustisectum, Ap. graveolens, Daucus carota, Foeniculum vulgare, Petroselinum crispum, Salix babylonica, S. viminalis. La cita de esta especie dada por Prado (1991) y por Klein Koch \& Waterhouse (2000) sobre Asparagus officinalis tuvo que deberse a especímenes errantes, como los recogidos sobre Solanum lycopersicon y Triticum de la

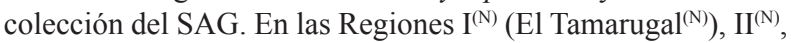
III, IV (Elqui), V (Marga Marga, Petorca, Quillota, San Felipe de Aconcagua, Valparaíso), M (Chacabuco ${ }^{(\mathrm{N})}$, Cordillera $\left.^{(\mathrm{N})}\right)$, VI (Cachapoal $\left.^{(\mathrm{N})}\right)$, VII (Cauquenes $^{(\mathrm{N})}$, Talca), VIII (Nuble), IX (Cautín, Malleco), XIV ${ }^{(\mathrm{N})}$ (Valdivia $\left.^{(\mathrm{N})}\right), \mathrm{X}$ (Osorno) y XI (Coihaique), XII $^{(\mathrm{N})}$ (Magallanes $\left.^{(\mathrm{N})}\right)$.

Chaetosiphon (Pentatrichopus) fragaefolii (Cockerell, 1901).Sobre Fragaria, F. chiloensis y F. x ananassa. En las Regiones III, IV, V (Marga Marga, Valparaíso), M (Santiago), VI, VII (Cauquenes, Talca), VIII (Arauco, Ñuble), IX y X (Chiloé, Llanquihue).

Chaetosiphon (Pentatrichopus) tetrarhodum (Walker, 1849).Sobre Rosa. En las Regiones V (Quillota) y VII ${ }^{(\mathrm{N})}\left(\mathrm{Talca}^{(\mathrm{N})}\right)$.

Chaetosiphon (Pentatrichopus) thomasi (Hille Ris Lambers, 1953).- Sobre Fragaria, Fragaria chiloensis y F. x ananassa (quizás a partir de identificaciones incorrectas de $C$. fragaefolii) y Rosa. En las Regiones II ${ }^{(\mathrm{N})}\left(\mathrm{El} \mathrm{Loa}^{(\mathrm{N})}\right)$, IX, XIV (Valdivia) y X.

Chaitophorus leucomelas (Koch, 1854).- Sobre Populus deltoides y P. nigra. En la Región XV (Arica), I (Iquique $\left.{ }^{(\mathrm{N})}\right)$, II, III, IV, V (Valparaíso $\left.^{(\mathrm{N})}\right)$, M (Santiago $\left.{ }^{(\mathrm{N})}\right)$, VI (Cachapoal), VII (Cauquenes, Curicó, Linares, Talca), VIII (Bío Bío) y IX (Cautín). 
Chromaphis juglandicola (Kaltenbach, 1843).- Sobre Juglans regia. En las Regiones IV (Choapa), V (Los Andes), M (Maipo, Talagante), VI (Cachapoal), VII, VIII.

Cinara cedri Mimeur, 1936.- Sobre Cedrus y Cedrus libani. Los ejemplares de esta especie de la colección del SAG recogidos sobre Larix decidua deben considerarse errantes. En las Regiones V (Quillota, Los Andes ${ }^{(\mathrm{N})}$ ), M (Melipilla ${ }^{(\mathrm{N})}$, Santiago $\left.^{(\mathrm{N})}\right)$ y IX ${ }^{(\mathrm{N})}\left(\right.$ Osorno $\left.^{(\mathrm{N})}\right)$.

Cinara (Cupressobium) cupressi (Buckton, 1881).Sobre Austrocedrus chilensis, Callitropsis macrocarpa, Chamaecyparis, Cupressus, Cu. sempervirens, Cupressaceae, Fitzroya cupressoides y Thuja. En las Regiones $\mathrm{XV}^{(\mathrm{N})}$ (Arica $\left.^{(\mathrm{N})}\right)$, I (El Tamarugal), II, III, IV, V (San Antonio $\left.{ }^{(\mathrm{N})}\right), \mathrm{M}$, VI, VII $\left(\right.$ Curicó $\left.^{(\mathrm{N})}\right)$, VIII, IX, XIV, X, XI y XII (Magallanes, Última Esperanza).

Cinara (Cupressobium) cupressivora Watson \& Voegtlin, 1999.- La validez taxonómica de esta especie no es admitida universalmente y además no es fácil separarla de $C$. cupressi (véase Blackman \& Eastop, 2016) e incluso de fresai; en consecuencia, cabe poner en duda las citas de esta especie (Forestry Department FAO, 2008). Sobre Austrocedrus chilensis. En las Regiones V, M, VI, VII, VIII, IX, X, XI.

Cinara (Cupressobium) fresai (Blanchard (E.E.), 1939).- Sobre Austrocedrus chilensis, Callitropsis macrocarpa, Cupressus ${ }^{(\mathrm{N})}$ y $C$. macrocarpa ${ }^{(\mathrm{N})}$. En las Regiones I, II ${ }^{(\mathrm{N})}\left(\mathrm{El} \mathrm{Loa}{ }^{(\mathrm{N})}\right)$, III \left.${\left(\text { Copiapó }^{(\mathrm{N})}\right), \mathrm{IV}^{(\mathrm{N})}\left(\text { Elqui }^{(\mathrm{N})}\right), \mathrm{V}\left(\text { San } \text { Antonio }^{(\mathrm{N})} \text {, Valparaíso }\right.}^{(\mathrm{N})}\right)$, M (Santiago ${ }^{(\mathrm{N})}$ ), VI, VII, VIII, IX, XIV (Valdivia), X y XI.

Cinara (Cupressobium) juniperi (De Geer, 1773).- Sin hospedadora. En la Región IX.

Cinara pilicornis (Hartig, 1841).- Sobre Picea. En las Regiones IX (Malleco), XIV (Valdivia), XI (Coihaique $^{(\mathrm{N})}$ ) y XII ${ }^{(\mathrm{N})}$ (Magallanes $\left.^{(\mathrm{N})}\right)$.

Cinara pinimaritimae (Dufour, 1833).- Citada en Chile, como en gran parte del Mundo, bajo el incorrecto nombre de Cinara maritimae. Sobre Pinus palustris ${ }^{(\mathrm{N})}$ y P. radiata $^{(\mathrm{N})}$. En las Regiones VII ${ }^{(\mathrm{N})}\left(\right.$ Linares $^{(\mathrm{N})}$, Talca $\left.{ }^{(\mathrm{N})}\right)$, VIII $\left(\right.$ Arauco $^{(\mathrm{N})}$, Nuble $\left.^{(\mathrm{N})}\right)$ y IX $\left(\right.$ Malleco $\left.^{(\mathrm{N})}\right)$.

Cinara (Cupressobium) tujafilina (Del Guercio, 1909).- Sobre Austrocedrus chilensis, Cupressus ${ }^{(\mathrm{N})}$ y Thuja ${ }^{(\mathrm{N})}$. En las Regiones I, II ${ }^{(\mathrm{N})}\left(\mathrm{El} \mathrm{Loa}^{(\mathrm{N})}\right), \mathrm{V}, \mathrm{M}$, VI $\left(\right.$ Cachapoal $^{(\mathrm{N})}$, Colchagua $\left.^{(\mathrm{N})}\right)$, VII, VIII, IX, X y XI.

Cryptomyzus ballotae Hille Ris Lambers, 1953.- Sobre Marrubium vulgare. En la Región V (Valparaíso).

Diuraphis noxia (Kurdjumov, 1913).- Sobre Avena sativa, Hordeum vulgare, Poaceae, Triticum y T. aestivum. En las Regiones $\mathrm{II}^{\mathrm{N})}\left(\mathrm{El} \mathrm{Loa}{ }^{(\mathrm{N})}\right)$, III, IV (Elqui, Limarí), V (Los Andes, Quillota, San Felipe de Aconcagua), M (Santiago), VI (Cachapoal, Colchagua), VII (Cauquenes, Curicó, Linares, Talca), VIII (Bío Bío, Nuble), IX (Cautín, Malleco), XIV (Valdivia) y XII (Magallanes).

Drepanosiphum oregonense Granovsky, 1939.- Sobre Acer y A. pseudoplatanus $^{(\mathrm{N})}$. En las Regiones X (Osorno) y $\mathrm{XI}^{(\mathrm{N})}$ $\left(\right.$ Coihaique $\left.^{(\mathrm{N})}\right)$.

Dysaphis apiifolia apiifolia Theobald, 1923.- Starý (1995) citó la especie sin mencionar subespecie y también $D$. apiifolia petroselini (Börner, 1950); de acuerdo con Blackman \& Eastop (2006) la subespecie nominotípica debe ser la única que se presenta en Chile, dado el ciclo vital de ambas subespecies, la cita de Muñoz (1995) y el material estudiado para la nueva cita. Sobre Apium graveolens, Conium maculatum y Foeniculum vulgare. En las Regiones $\mathrm{I}^{\mathrm{N})}$ (El Tamarugal $\left.{ }^{(\mathrm{N})}\right)$, IV (Elqui), V (Quillota) y VIII (Arauco, Nubble ${ }^{(\mathrm{N})}$ ).

Dysaphis foeniculus (Theobald, 1923).- Sobre Apium graveolens y Daucus carota. En las Regiones $\mathrm{XV}^{(\mathrm{N})}\left(\right.$ Arica $\left.^{(\mathrm{N})}\right)$, VI (Cachapoal) y XIV (Valdivia).
Dysaphis lappae cynarae (Theobald, 1915).- Sobre Cynara cardunculus. En las Regiones III, IV, V, M $\left(\right.$ Santiago $\left.^{(\mathrm{N})}\right)$, VI, VII, VIII, IX, XIV, X, XII ${ }^{(\mathrm{N})}$ (Magallanes $\left.^{(\mathrm{N})}\right)$. La subespecie D. lappae lappae (Koch, 1854) no ha sido citada en Chile.

Dysaphis tulipae (Boyer de Fonscolombe, 1841).- Sobre Iris, I. germanica, Lilium y Tulipa. En las Regiones V (Valparaíso) y $\mathrm{M}$.

Elatobium abietinum (Walker, 1849).- Sobre Pseudotsuga menziesi, Picea. En las Regiones IX, XIV (Valdivia) y X, XI ${ }^{(\mathbb{N})}$ Coihaique $\left.^{(\mathrm{N})}\right)$ y XII ${ }^{(\mathrm{N})}$ (Magallanes $\left.^{(\mathrm{N})}\right)$.

Eriosoma lanigerum (Haussman, 1802).- Sobre Malus domestica, Pyrus communis y Pyracantha coccinea. En las Regiones I, II, III (Copiapó $\left.{ }^{(\mathrm{N})}\right)$, IV, V (Marga Marga), M (Santiago), VI (Cachapoal, Colchagua), VII (Curicó, Linares, Talca), VIII (Bío Bío, Nuble ${ }^{(\mathrm{N})}$ ), IX (Cautín, Malleco), XIV, X y XI

Eriosoma (Mimaphidus) pyricola Baker \& Davidson, 1916.Sobre Cydonia oblonga, Pyrus communis y Ulmus. En las Regiones V, M (Santiago), VI (Cachapoal ${ }^{(\mathbb{N})}$ ), VII (Curicó), VIII, IX, XIV y X.

Essigella californica (Essig, 1909).- Sobre Pinus ${ }^{(\mathrm{N})}$ y P. radia$t a^{(\mathrm{N})}$. En las Regiones V, M (Santiago $\left.{ }^{(\mathrm{N})}\right)$, VI $\left(\right.$ Cachapoal $\left.^{(\mathrm{N})}\right)$ y VII $\left(\right.$ Curicó $\left.^{(\mathrm{N})}\right)$.

Eucarazzia elegans (Ferrari, 1872).- Sobre Phrodus microphyllus, Pitraea cuneato-ovata ${ }^{(\mathrm{N})}$. En las regiones $\mathrm{XV}^{(\mathrm{N})}\left(\right.$ Arica $\left.^{(\mathrm{N})}\right)$, IV (Elqui), VI ${ }^{(\mathrm{N})}$ (Cachapoal $\left.^{(\mathrm{N})}\right)$. Las dos plantas mencionadas, respectivamente solanácea y verbenácea, son plantas sorprendentes para la especie, que hasta ahora solamente había sido citada sobre especies de Lamiaceae.

Eulachnus rileyi (Williams, 1911).- Sobre Pinus, P. contorta, $P$. ponderosa y $P$. radiata. En las Regiones $\mathrm{V}^{(\mathrm{N})}\left(\right.$ Marga Marga $\left.^{(\mathrm{N})}\right)$, $\mathrm{M}^{(\mathrm{N})}\left(\right.$ Santiago $\left.^{(\mathrm{N})}\right)$, VIII (Nuble), IX ${ }^{(\mathrm{N})}\left(\right.$ Cautín $\left.^{(\mathrm{N})}\right)$ y X.

Geoica lucifuga (Zehntner, 1897).- Sobre Agrostis capillaris, Arrhenatherum elatius, Lolium, Lotus, Notholcus lanatus, Poaceae, Trifolium repens y Tr. subterraneum. En la Región XIV (Valdivia).

Hoplocallis picta (Ferrari, 1872).- Smith \& Cermeli (1979) y Blackman \& Eastop (2016) mencionan Chile en la distribución de esta especie, pero no se ha podido localizar la fuente bibliográfica en la que se apoyaron. Sobre Quercus $^{(\mathrm{N})}$. En $\mathrm{V}^{(\mathrm{N})}$ (Valparaíso $\left.^{(\mathrm{N})}\right)$ y $\mathrm{M}^{(\mathrm{N})}\left(\right.$ Santiago $\left.^{(\mathrm{N})}\right)$.

Hyadaphis foeniculi (Passerini, 1860).- Los especímenes de $H$. foeniculi o de Hyadaphis passserinii (del Guercio) se pueden identificar sin dificultad como pertenecientes alguna de estas dos especies, pero la identificación específica concreta es difícil y además insegura si sólo se dispone de unos pocos ejemplares preparados. Essig (1953) citó esta especie en Chile sobre una especie de Carum, género sobre el que $H$. passerinii no se ha citado hasta el momento (Holman, 2009; Blackman \& Eastop, 2016). Las citas de esta especie, sin localidades concretas de las regiones V, M, VI y VII, de Prado (1991) deben de ponerse en duda, pues se basan en especímenes colectados sobre Daucus carota que según Blackman \& Eastop (2016) es hospedadora posible pero infrecuente para $H$. foeniculi y en cambio es hospedadora reconocida para $H$. passerinii. La cita de la especie por Zúñiga (1967a) en la provincia de Quillota (Región V) es también dudosa por estar basada en una única hembra vivípara alada recogida accidentalmente sobre Solanum lycopersicon. Sobre Carum. En la Región V (Los Andes).

Hyadaphis passerinii (Del Guercio, 1911).- Primeras citas en Chile de esta especie paleártica a partir de especímenes conservados en las colecciones del SAG, de las universidades de León y de Talca y de la J. Ortego. Ya estaba citada en América del Sur, pero no en los países limítrofes (Blackman \& Eastop, 2016). Sobre Apiaceae, Daucus carota, Foeniculum vulgare y Lonicera. En las Regiones V (San Felipe de Aconcagua), M (Santiago) 
[aunque con dudas], VI (Cachapoal), VII (Linares) [aunque con dudas], X (Osorno) y XII (Punta Arenas). Además es posible que se puedan asignar a esta especie las citas que Zúñiga (1967a) y Prado (1991) dan para H. foeniculi (véase su entrada) en las regiones V, M, VI y VII.

Hyalopterus pruni (Geoffroy, 1762).- Sobre Prunus domestica. La única cita bibliográfica de primera mano de esta especie (bajo el nombre de Hyalopterus arundinis sobre «prune trees») se debe a Essig (1953), que no precisó localización ninguna. En las Regiones III ${ }^{(\mathrm{N})}\left(\right.$ Copiapó $\left.^{(\mathrm{N})}\right)$ y VIII ${ }^{(\mathrm{N})}\left(\mathrm{N}\right.$ uble $\left.{ }^{(\mathrm{N})}\right)$.

Hyperomyzus carduellinus (Theobald, 1915).- Primera cita en Chile a partir de especímenes conservados en la colección de la Universidad de León de esta especie posiblemente originaria del este paleártico y que ya es conocida en Argentina (Ortego et al., 2004). Sobre Sonchus oleraceus. En la Región VII (Talca).

Hyperomyzus lactucae (Linnaeus, 1758).- Sobre Acanthus mollis, Lactuca sativa, Sonchus, S. asper y S. oleraceus. La cita de esta especie sobre Chrysanthemum debida a Starý $(1994,1995)$ y los de la colección del SAG recogidos sobre Medicago sativa deben considerarse errantes. En las Regiones $\mathrm{XV}^{(\mathrm{N})}\left(\right.$ Arica $\left.^{(\mathrm{N})}\right)$, $\mathrm{I}^{(\mathrm{N})}\left(\right.$ El Tamarugal $\left.{ }^{(\mathrm{N})}\right), \mathrm{II}^{(\mathrm{N})}\left(\mathrm{El} \mathrm{Loa}^{(\mathrm{N})}\right), \mathrm{V}$ (Petorca, Quillota), M, VI, VII, VIII (Ñuble), IX, XIV, X (Llanquihue $\left.{ }^{(\mathrm{N})}\right)$, XI y XII.

Hysteroneura setariae (Thomas, 1878).- Sobre Stipa speciosa. En las Regiones IV (Elqui) y V (Isla de Pascua).

Idiopterus nephrelepidis Davis, 1909.- Sobre helechos no identificados. En la Región $\mathrm{M}^{(\mathrm{N})}$ (Santiago $\left.{ }^{(\mathrm{N})}\right)$ y VIII (Ñuble).

Illinoia azaleae (Mason, 1925).- Primera cita en Chile de esta especie neártica a partir de especímenes conservados en la colección del SAG; ya es conocida en Argentina (Ortego et al., 2004). Sobre Rhododendron. En la Región M (Santiago).

Illinoia (Masonaphis) lambersi (MacGillivray, 1960).- Sobre Rhododendron japonicum. Las citas de Prado (1991) y de Klein Koch \& Waterhouse (2000) de este pulgón sobre arándano cultivado en las Regiones IX y X son dudosas, porque no vive sobre arándano, mientras que otras especies del género sí lo hacen. En la Región XIV (Valdivia).

Illinoia morrisoni (Swain, 1918).- Sobre Cupressus $^{(\mathrm{N})}$. En las Regiones I (El Tamarugal $^{(\mathrm{N})}$, Iquique $\left.{ }^{(\mathrm{N})}\right)$, II, III, IV, V, M, VI, VII, VIII, IX, XIV y X.

Illinoia pepperi (MacGillivray, 1958).- Primera cita en Chile de esta especie neártica a partir de especímenes conservados en la colección del SAG. Sobre Vaccinium. En la Región V (Valparaíso). Ésta es también su primera cita en América del Sur.

Lipaphis pseudobrassicae (Davis, 1905).- Existe consenso (Blackman \& Eastop, 2016) en que Lipaphis erysimi está restringida a Europa y que las citas con ese nombre en otras partes del Mundo deben asignarse a L. pseudobrassicae. Sobre Brassica napus, Hirschfeldia incana y Matthiola incana. En las Regiones I ${ }^{(\mathrm{N})}$ (Iquique $\left.^{(\mathrm{N})}\right)$, III ${ }^{(\mathrm{N})}\left(\operatorname{Huasco}^{(\mathrm{N})}\right)$, IV $\left(\right.$ Choapa $^{(\mathrm{N})}$, Elqui), VII ${ }^{(\mathbb{N})}\left(\right.$ Curicó $\left.^{(\mathrm{N})}\right)$, VIII $^{(\mathbb{N})}\left(\right.$ Nuble $\left.^{(\mathrm{N})}\right)$, IX (Cautín) y XIV (Valdivia).

Macrosiphoniella sanborni (Gillette, 1908).- $\quad$ Sobre Chrysanthemum y C. morifolium. En las Regiones V (Quillota), $\mathrm{M}^{\mathrm{N})}\left(\right.$ Santiago $\left.^{(\mathrm{N})}\right)$, XIV (Valdivia) y X.

Macrosiphoniella tanacetaria bonariensis (Blanchard, 1922).Sobre Asteraceae. En la Región VIII (Ñuble). La subespecie $M$. tanacetaria tanacetaria (Kaltenbach, 1843) no ha sido citada en Chile.

Macrosiphum euphorbiae (Thomas, 1878).- Sobre Acanthus mollis, Acca sellowiana, Actinidia chinensis var. deliciosa, Alstroemeria leporina, Anethum graveolens, Asparagus officinalis, Beta vulgaris, Brassica oleracea var. capitata, Br. oleracea var. viridis, Canna, Capsicum annuum, Cap. frutescens, Cereus, Chenopodium, Citrus, Ci. limon, Ci. maxima, Convolvulus, Cristaria ${ }^{(\mathrm{N})}$, Cucumis sativus, Cucurbita ficifolia,
Cucur. maxima, Cynara cardunculus, Eriobotrya japonica ${ }^{(\mathrm{N})}$, Fumaria officinalis, Gamochaeta spiciforme ${ }^{(\mathrm{N})}$, Helianthus annuus, Helminthotheca echioides, Iris, Lactuca sativa, Lathyrus sativus, Oxalis pes-caprae, Papaver, Phaseolus, Ph. vulgaris, Pistacia vera, Pisum sativum, Pluchea absinthioides, Punica granatum ${ }^{(\mathrm{N})}$, Robinia pseudoacacia ${ }^{(\mathrm{N})}$, Ruta bracteosa, Silybum marianum ${ }^{(\mathrm{N})}$, Simmondsia chinensis, Solanum lycopersicon, Sol. melongena, Sol. muricatum, Sol. tuberosum, Sonchus, Son. asper, Spiraea cantoniensis ${ }^{(\mathrm{N})}$, Trifolium, Triticum aestivum, Tulipa, Veronica anagallis-aquatica, Vicia faba y Zea mays. En las Regiones XV (Arica), I (Iquique ${ }^{(\mathrm{N})}$ ), II (Antofagasta, El Loa), III, IV (Elqui), V (Los Andes, Marga Marga, Petorca, Quillota, San Felipe de Aconcagua, Valparaíso $\left.^{(\mathrm{N})}\right)$, M (Cordillera $^{(\mathrm{N})}$, Santiago), VI $\left(\right.$ Cachapoal $\left.^{(\mathrm{N})}\right)$, Colchagua $\left.^{(\mathrm{N})}\right)$, VII, VIII (Bío Bío ${ }^{(\mathrm{N})}$, Concepción ${ }^{(\mathrm{N})}$, Nuble), IX (Cautín, Malleco), XIV, X (Llanquihue, Osorno), XI (Coihaique $^{(\mathrm{N})}$ ) y XII.

Macrosiphum rosae (Linnaeus, 1758).- Sobre Rosa, R. moschata $^{(\mathrm{N})}$. En las Regiones I, II, III, IV (Elqui), V (Los Andes, Quillota, Valparaíso), M (Santiago), VI, VII $\left(\right.$ Talca $\left.^{(\mathrm{N})}\right)$, VIII (Ñuble), IX (Cautín), XIV (Valdivia), X, XI (General Carrera) y XII ${ }^{(\mathrm{N})}$ (Magallanes $\left.^{(\mathrm{N})}\right)$.

Melanaphis donacis (Passerini, 1862).- Sobre Arundo donax y Chusquea. En las Regiones XV (Arica), I (Iquique $^{(\mathrm{N})}$ ), \left. III ${\left(\text { Copiapó }^{(\mathrm{N})}, \text { Huasco }^{(\mathrm{N})}\right) \text {, V } \text { (Quillota }^{(\mathrm{N})} \text {, Valparaíso }}^{(\mathrm{N})}\right)$, M (Talagante) y VI (Cachapoal).

Metopolophium dirhodum (Walker, 1849).- Sobre Agrostis verticillata, Avena, Av. fatua, Av. sativa, Bromus, B. berteroanus, B. catharticus, B. hordeaceus, B. rigidus, Capriola dactylon, Dactylis, D. glomerata, Elymus angulatus, Festuca, Hordeum, H. chilense, H. murinum, H. vulgare, Lolium, L. perenne, Oryza sativa, Phalaris, Ph. amethystina, Ph. canariensis, Ph. minor, Poa pratensis, Poaceae, Polypogon, Pol. lagascae, Pol. linearis, Secale cereale, Sisyrinchium, Si. graminifolium, Stipa, Triticum, T. aestivum, Vulpia megalura y Zea mays. La cita de esta especie dada por Starý et al. (1994a) sobre Papaver debe considerarse accidental. En las Regiones I, II (El Loa $\left.{ }^{(\mathrm{N})}\right)$, III (Huasco), IV (Elqui, Limari $^{(\mathrm{N})}$ ), V (Los Andes, Petorca, Quillota, San Felipe de Aconcagua, Valparaíso), M (Cordillera, Melipilla, Santiago), VI (Cachapoal, Cochagua, Cardenal Caro), VII (Cauquenes, Curicó, Linares, Talca), VIII (Arauco, Bío Bío, Nuble), IX (Cautín, Malleco), XIV (Valdivia), X y XI ${ }^{(\mathrm{N})}\left(\right.$ General Carrera $\left.^{(\mathrm{N})}\right)$.

Metopolophium festucae cerealium Stroyan, 1962.- Sobre Avena sativa, Hordeum vulgare, Poaceae y Triticum aestivum. En las Regiones V (Quillota), VII (Cauquenes, Linares, Talca), VIII (Arauco, Bío Bío, Nuble) y IX (Cautín, Malleco). La subespecie M. festucae festucae (Theobald, 1917) no ha sido citada en Chile.

Microlophium carnosum (Buckton, 1876).- Sobre Urtica. En la Región XIV (Valdivia).

Myzaphis rosarum (Kaltenbach, 1843).- Sobre Rosa. En las Regiones $\mathrm{XV}^{(\mathrm{N})}\left(\operatorname{Parinacota}^{(\mathrm{N})}\right), \mathrm{M}\left(\right.$ Santiago $\left.^{(\mathrm{N})}\right)$, VI, VII, VIII, IX $\left(\right.$ Cautín $\left.{ }^{(\mathrm{N})}\right)$ y X.

Myzocallis boerneri Stroyan, 1957.- Sobre Quercus ilex, Q. cerris, Q. suber. En las Regiones V, M (Santiago), VI (Cachapoal) y VIII.

Myzocallis (Agrioaphis) castanicola castanicola Baker, 1917.Citada en Chile sin precisar subespecie; se trata sin duda de la nominotípica. Sobre Castanea sativa, Quercus, Q. nigra ${ }^{(\mathbb{N})}$ y Q. suber $^{(\mathrm{N})}$. En las Regiones V (Valparaíso ${ }^{(\mathrm{N})}$, Quillota $\left.^{(\mathrm{N})}\right), \mathrm{M}^{(\mathrm{N})}$ $\left(\right.$ Santiago $\left.^{(\mathrm{N})}\right)$, VI, VIII ${ }^{(\mathrm{N})}\left(\right.$ Nuble $\left.^{(\mathrm{N})}\right)$ y XIV (Valdivia).

Myzocallis coryli (Goeze, 1778).- Sobre Corylus avellana. En las Regiones IV, VI (Colchagua $^{(\mathrm{N})}$ ), VII (Cauquenes $^{(\mathrm{N})}$, Curicó, Linares, Talca) ${ }^{(\mathrm{N})}$, VIII, IX (Cautín, Malleco $\left.{ }^{(\mathrm{N})}\right)$, XIV (Valdivia) y X. 
Myzus (Sciamyzus) ascalonicus Doncaster, 1946.- Sobre Viola En las Regiones XIV (Valdivia) y XI ${ }^{(\mathrm{N})}\left(\right.$ Coihaique $\left.^{(\mathrm{N})}\right)$.

Myzus (Sciamyzus) cymbalariae Stroyan, 1954.- Sobre Oxalis laxa. En la Región V (Quillota).

Myzus ornatus Laing, 1932.- Sobre Bidens aurea, Bignonia, Carica, Ca.papaya, Cereus, Cichorium intybus, Cucurbita ficifolia, Fuchsia magellanica, Gladiolus, Glycine max, Gunnera magellanica, Hebe salicifolia, Helianthus annuus, Lactuca sativa, Lathyrus magellanicus, Malus, Malus domestica, Malva $^{(\mathrm{N})}$, Medicago sativa, Petroselinum crispum $^{(\mathrm{N})}$, Plantago major, Rosa ${ }^{(\mathbb{N})}$, Senecio smithii, Sparmannia ${ }^{(\mathbb{N})}$, Trifolium, T. pratense, T. repens, Verbena littoralis y Vicia sativa. En las Regiones XV (Arica), I (Iquique $\left.{ }^{(\mathrm{N})}\right)$, II (Antofagasta, El Loa ${ }^{(\mathrm{N})}$ ), III, IV, V (Quillota, San Felipe de Aconcagua, Valparaíso $\left.{ }^{(\mathrm{N})}\right), \mathrm{M}$ (Santiago), VI (Cachapoal $\left.{ }^{(\mathrm{N})}\right)$, VII $\left(\right.$ Linares $\left.^{(\mathrm{N})}\right)$, VIII (Nuble), IX (Cautín $^{(\mathrm{N})}$, Coihaique), XIV (Valdivia) y X.

Myzus (Nectarosiphon) persicae nicotianae Blackman, 1987.Esta subespecie fue detectada en Chile en 2000 (FuentesContreras et al., 2004). Posiblemente las citas de Zúñiga (1967a, 1968), Zúñiga \& Suzuki (1976) y Prado (1991) sobre plantas de tabaco en la Región V correspondan a M. persicae persicae. Sobre Nicotiana tabacum. En Regiones V (San Felipe de Aconcagua), VI (Cachapoal, Colchagua, Cardenal Caro) y VII (Curicó, Linares, Talca) y VIII (Ñuble).

Myzus (Nectarosiphon) persicae persicae (Sulzer, 1776).Casi todas las citas de esta especie se han dado sin concretar subespecie; se puede admitir sin problemas que la subespecie implicada en casi todos los casos sea la nominotípica (ver M. persicae nicotianae). Sobre Acca sellowiana, Actinidia chinensis var. deliciosa, Amygdalus communis, Anoda, An. cristata, Asparagus officinalis, Asteraceae, Avena fatua, Baccharis linearis ${ }^{(\mathrm{N})}$, Beta vulgaris, Be. vulgaris subsp. cicla, Brassica, Bra. napus, Bra. oleracea, Bra. oleracea var. botrytis, Bra. oleracea var. capitata, Bra. oleracea var. gemmifera, Bra. oleracea var. viridis , Bra. rapa, Bromus, Calendula officinalis, Capsella bursa-pastoris, Capsicum annuum, Capsi. frutescens, Carica pubescens, Carpobrotus chilensis,

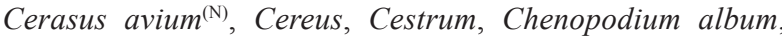
Chrysanthemum, Chr. coronarium, Chr. morifolium, Citrus, Ci. limon, Ci. maxima, Convolvulus, Co. arvensis, Cucumis melo, Cucurbita ficifolia, Cucur. maxima, Cynara, Datura, Da. stramonium, Dianthus, Erodium cicutarium, Euphorbia peplus, Eu. pulcherrima, Helianthus annuus, Hibiscus, Hirschfeldia incana, Hordeum murinum, Ho. vulgare, Ipomea batatas, Juglans regia, Lactuca sativa, Lamiaceae ${ }^{(\mathrm{N})}$, Ligustrum, Malva, Ma. nicaensis, Medicago sativa, Mesembryanthemum, Nicotiana tabacum, Oxalis, Papaver, Persea americana ${ }^{(\mathrm{N})}$, Persicaria maculosa, Petroselinum, Pet. crispum, Phaseolus vulgaris, Pistacia vera, Pisum sativum, Poaceae, Prunus, Pr. armeniaca, Pr. domestica, Pr. persica, Pr. salicina, Punica granatum, Raphanus, Ra. raphanistrum, Ra. sativus, Rumex, Senecio bicolor subsp. cineraria, Simmondsia chinensis, Solanum, So. lycopersicon, So. melongena, So. muricatum, So. tuberosum, Trifolium, Triticum aestivum, Tulipa, Ulmus, Urtica urens, Vicia faba y Viola odorata. En las Regiones XV (Arica), I, II (Antofagasta, El Loa), III (Copiapó, Huasco), IV (Elqui), V (Isla de Pascua, Los Andes, Marga Marga, Petorca, Quillota, San Felipe de Aconcagua, Valparaíso), M (Maipó, Melipilla, Santiago), VI (Cachapoal, Colchagua), VII (Cauquenes, Curicó, Talca ${ }^{(\mathrm{N})}$ ), VIII (Arauco, Bío Bío, Nuble), IX (Malleco), XIV (Ranco, Valdivia), X (Llanquihue, Osorno), XI y XII (Magallanes $\left.{ }^{(\mathrm{N})}\right)$.

Nasonovia ribisnigri (Mosley, 1841).- Sobre Asteraceae, Cichorium intybus, Hieracium $^{(\mathrm{N})}$, Lactuca, L. sativa, Sonchus asper y S. oleraceus. En las Regiones IV (Elqui), V (Los
Andes, Quillota), $\mathrm{M}^{(\mathrm{N})}$ (Chacabuco ${ }^{(\mathrm{N})}$, Cordillera $\left.^{(\mathrm{N})}\right), \mathrm{VI}^{(\mathrm{N})}$ $\left(\right.$ Cachapoal $\left.^{(\mathrm{N})}\right)$, VII, VIII (Bío Bío ${ }^{(\mathrm{N})}$, Nuble), IX ${ }^{(\mathrm{N})}\left(\right.$ Cautín $\left.^{(\mathrm{N})}\right)$, $\mathrm{XIV}^{(\mathrm{N})}\left(\right.$ Ranco $\left.^{(\mathrm{N})}\right), \mathrm{XI}^{(\mathrm{N})}\left(\right.$ Coihaique $\left.^{(\mathrm{N})}\right)$ y XII ${ }^{(\mathrm{N})}$ (Magallanes $^{(\mathrm{N})}$, Tierra del Fuego $\left.{ }^{(\mathrm{N})}\right)$.

Nearctaphis bakeri (Cowen, 1895).- Sobre Medicago sativa y Trifolium repens. En las Regiones V (Quillota), M (Santiago) y XI ${ }^{(\mathrm{N})}\left(\right.$ Cautín $\left.^{(\mathrm{N})}\right)$.

Neomyzus circunflexus (Buckton, 1876).- Sobre Glycine max y Solanum tuberosum. En las Regiones V (Quillota), IX y X (Osorno).

Neophyllaphis (Chileaphis) michelbacheri (Essig, 1953).- Sobre Pilgerodendron uviferum. Es posible que la presencia de esta especie sobre $P$. uviferum fuera accidental, y que su planta hospedadora real sea alguna especie de Podocarpus. En la Región $\mathrm{X}$ (Llanquihue).

Neophyllaphis (Chileaphis) podocarpini (Carrillo, 1980).- Sobre Podocarpus salignus. En la Región XIV (Valdivia).

Neosensoriaphis parva Quednau, 1990.- Sobre Nothofagus macrocarpa, N. obliqua. En las Regiones M (Santiago) y IX (Cautín, Malleco)

Neotoxoptera formosana (Takahashi, 1921).- Sobre Allium cepa y A. porrum. En las Regiones V (Quillota), M (Santiago), VI ${ }^{(\mathbb{N})}$ $\left(\right.$ Cachapoal $\left.^{(\mathrm{N})}\right)$ y XII ${ }^{(\mathrm{N})}$ (Magallanes $\left.^{(\mathrm{N})}\right)$.

Neotoxoptera oliveri (Essig, 1935). - Primera cita en Chile de esta especie, de distribución prácticamente cosmopolita y ya citada en el Perú, a partir de especímenes conservados en la colección del SAG. Sobre Allium schoenoprasum. En la Región M (Santiago).

Neuquenaphis bulbicauda Hille Ris Lambers, 1968.- Sobre Nothofagus, $N$. dombeyi y quizás accidental sobre $N$. obliqua. En las Regiones VII (Talca), VIII (Arauco, Ñuble), IX (Cautín, Malleco), XIV (Valdivia), X (Chiloé, Osorno) y XII (Última Esperanza).

Neuquenaphis (Spicaphis) chilensis Essig, 1953.- Sobre Nothofagus dombeyi, N. nitida y N. obliqua. En las Regiones VIII (Arauco ${ }^{(\mathrm{N})}$, Nuble), IX (Cautín), X (Llanquihue, Osorno) y XI (Coihaique).

Neuquenaphis edwardsi (Laing, 1927).- Sobre Nothofagus, $N$. alpina, $N$. antarctica, $N$. betuloides, $N$. dombeyi, $N$. glauca, $N$. macrocarpa, N. obliqua y N. pumilio. En las Regiones V (Marga Marga), $\mathrm{M}^{(\mathrm{N})}$ (Santiago $\left.^{(\mathrm{N})}\right), \mathrm{VII}^{(\mathrm{N})}$ (Cauquenes $^{(\mathrm{N})}$, Curicó $\left.^{(\mathrm{N})}\right)$, VIII $\left(\right.$ Arauco $^{(\mathrm{N})}$, Bío Bío ${ }^{(\mathrm{N})}$, Nuble), IX (Cautín, Malleco), XIV (Valdivia, El Ranco), X (Chiloé(N), Llanquihue, Osorno), XI ${ }^{(\mathrm{N})}$ (Coihaique $^{(\mathrm{N})}$ ) y XII (Antártica Chilena, Magallanes, Tierra del Fuego).

Neuquenaphis (Spicaphis) essigi Hille Ris Lambers, 1968.Sobre Nothofagus dombeyi, N. glauca, N. macrocarpa, N. obliqua. En las Regiones V (Marga Marga), M (Santiago), VII (Talca) y VIII ${ }^{(\mathrm{N})}\left(\right.$ Arauco $\left.^{(\mathrm{N})}\right)$.

Neuquenaphis michelbacheri Hille Ris Lambers, 1968.- Sobre Nothofagus alessandrii, $N$. alpina, $N$. antarctica, N. dombeyi, N. glauca, N. macrocarpa, N. obliqua. En las Regiones V (Marga Marga), VII (Cauquenes, Talca), VIII (Ñuble), IX (Cautín, Malleco), XIV (Valdivia) y X (Osorno).

Neuquenaphis neobulbicauda Remaudière \& Quednau, 1994.Sobre Nothofagus dombeyi. En la Región X (Osorno).

Neuquenaphis palliceps Hille Ris Lambers, 1968.- Sobre Nothofagus, $N$. antarctica, $N$. dombeyi, $N$. glauca, $N$. nitida y N. obliqua En las Regiones $\mathrm{M}^{(\mathrm{N})}$ (Santiago $\left.^{(\mathrm{N})}\right)$, VII (Talca), VIII (Ñuble), IX (Cautín, Malleco), X (Chiloé(N), Llanquihue, Osorno), XI ${ }^{(\mathrm{N})}\left(\right.$ Coihaique $\left.^{(\mathrm{N})}\right)$ y XII (Última Esperanza).

Neuquenaphis schlingeri Hille Ris Lambers, 1968.- Sobre Nothofagus alessandrii, $N$. dombeyi, N. glauca, N. nitida, $N$. obliqua, En las Regiones VII (Cauquenes, Talca), VIII (Ñuble), IX (Cautín, Malleco), X y XI (Coihaique) 
Neuquenaphis sensoriata Hille Ris Lambers, 1968.- Sobre Nothofagus alessandrii, N. alpina, N. antartica, N. dombeyi, N. glauca, N. macrocarpa y N. obliqua. En las Regiones V

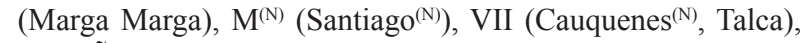
VIII (Nuble), IX (Malleco) y X.

Neuquenaphis similis Hille Ris Lambers, 1968.- Sobre Nothofagus antartica, N. obliqua y N. pumilio. En las Regiones VII (Talca), VIII (Arauco, Ñuble), IX (Malleco), X (Osorno), XI (Coihaique) y XII ${ }^{(\mathbb{N})}$ (Magallanes $\left.^{(\mathrm{N})}\right)$.

Neuquenaphis staryi Remaudière \& Quednau, 1994.- Sobre Nothofagus alessandrii. En la Región VII (Cauquenes).

Neuquenaphis valdiviana Carrillo, 1980.- Sobre Gunnera, G. magellanica, G. tinctoria y Nothofagus dombeyi. En las Regiones XIV (Valdivia) y XI (Coihaique).

Ovatus crataegarius (Walker, 1850).- Sobre Cydonia oblonga, Malus, M. domestica y Origanum vulgare. En las Regiones V, M (Santiago $\left.{ }^{(\mathrm{N})}\right)$, VI, VIII (Ñuble) y IX.

Pemphigus bursarius (Linnaeus, 1758).- Sobre Lactuca, L. sativa, Populus deltoides, P. nigra y Taraxacum officinale. En las Regiones V, M, VI (Cachapoal $\left.{ }^{(\mathbb{N})}\right)$, IX, XIV y X.

Pemphigus populitransversus Riley, 1879.- Sobre Populus nigra. En la Región III ${ }^{(\mathrm{N})}$ (Copiapó $\left.^{(\mathrm{N})}\right)$ y M (Santiago).

Pentalonia nigronervosa Coquerel, 1859.- Primera cita en Chile a partir de especímenes conservados en la colección del SAG. Sobre Musa x paradisiaca. En la Región I (Tarapacá). Especie originaria de la región Oriental y con muy amplia distribución en zonas con climas tropicales y subtropicales. Conocida en la Argentina y el Perú.

Phloeomyzus passerinii (Signoret, 1875).- Los caracteres que Capdeville (1945) da para unos pulgones observados en los troncos de los álamos a los que denomina Aphis populi, son los de $P$. passerinii. Sobre Populus nigra. En las Regiones $\mathrm{XV}^{(\mathrm{N})}\left(\right.$ Arica $\left.^{(\mathrm{N})}\right)$, II $^{(\mathrm{N})}\left(\right.$ Huasco $\left.^{(\mathrm{N})}\right)$, III $\left(\right.$ Copiapó $\left.^{(\mathrm{N})}\right)$, IV, V, M, VI $\left(\right.$ Cachapoal $\left.^{(\mathrm{N})}\right)$ y VII (Linares), $\mathrm{X}^{(\mathrm{N})}$ (Cautín $^{(\mathrm{N})}$ ) y XI ${ }^{(\mathrm{N})}\left(\right.$ General $\left._{\text {Carrera }}{ }^{(\mathrm{N})}\right)$.

Pleotrichophorus chrysanthemi (Theobald, 1920).- Sobre Chrysanthemum. En la Región V (Quillota).

Prociphilus fraxinifolii (Riley, 1879).- Sobre Fraxinus. En las Regiones VI ${ }^{(\mathrm{N})}\left(\right.$ Cachapoal $^{(\mathrm{N})}$ ) y VIII (Nuble).

Pterocallis alni (De Geer, 1773).- Sobre Alnus cordata y A. glutinosa. En las Regiones VI ${ }^{(\mathrm{N})}\left(\right.$ Cachapoal $\left.^{(\mathrm{N})}\right), \mathrm{VII}^{(\mathrm{N})}\left(\right.$ Linares $\left.^{(\mathrm{N})}\right)$, VIII $^{(\mathrm{N})}\left(\right.$ Nuble $\left.^{(\mathrm{N})}\right)$ y XIV (Valdivia).

Pterocomma populeum (Kaltenbach, 1843).- Sobre Populus nigra. Los ejemplares de la colección del SAG recogidos sobre Betula pendula deben considerarse errantes. En las Regiones $\mathrm{M}^{(\mathrm{N})}\left(\right.$ Cordillera $\left.^{(\mathrm{N})}\right)$, VI ${ }^{(\mathrm{N})}$ (Cachapoal $^{(\mathrm{N})}$ ), IX (Cautín), XIV (Valdivia) y XI ${ }^{(\mathrm{N})}$ (Coihaique ${ }^{(\mathrm{N})}$ ).

Rhodobium porosum (Sanderson, 1900).- Sobre Fragaria $\mathrm{x}$ ananassa y Rosa. En las Regiones V (Quillota) y M (Santiago).

Rhopalosiphoninus latysiphon (Davidson, 1912).- Sobre Phaseolus vulgaris y Solanum tuberosum. En las Regiones IV ${ }^{(\mathrm{N})}$ (Elqui $\left.^{(\mathrm{N})}\right), \mathrm{M}\left(\right.$ Santiago), VII ${ }^{(\mathrm{N})}\left(\right.$ Talca $\left.^{(\mathrm{N})}\right), \mathrm{VIII}^{(\mathrm{N})}\left(\right.$ Concepción $\left.^{(\mathrm{N})}\right)$ y IX ${ }^{(\mathrm{N})}\left(\right.$ Malleco $\left.^{(\mathrm{N})}\right)$.

Rhopalosiphum maidis (Fitch, 1856).- Sobre Arundo donax, Avena, Av. sativa, Echinochloa, E. crus-galli, Hordeum vulgare, Panicum, Poaceae, Setaria, Sorghum, So. bicolor, So. halepense, Triticum, T. aestivum y Zea mays. Las citas de esta especie sobre Nicotiana tabacum y Phaseolus vulgaris dadas por Zúñiga (1967b) deben ser consideradas accidentales. En las Regiones XV (Arica), I, II (El Loa, Huasco $\left.{ }^{(\mathrm{N})}\right)$, III (Copiapó), IV (Elqui, Limari $\left.{ }^{(\mathrm{N})}\right)$, V (Isla de Pascua, Los Andes, Marga Marga, Petorca, Quillota, San Felipe de Aconcagua), M (Cordillera, Santiago), VI (Cachapoal), VII, VIII (Ñuble ${ }^{(\mathrm{N})}$ ), IX, XIV y X.

Rhopalosiphum nymphaeae (Linnaeus, 1761).- Sobre Alisma y Nymphaea alba. La cita de esta especie sobre Phaseolus vulgaris dada por Zúñiga (1967b) debe ser considerada accidental. En las Regiones $\mathrm{II}^{(\mathrm{N})}\left(\mathrm{El} \mathrm{Loa}{ }^{(\mathrm{N})}\right) \mathrm{V}$ (Quillota), M (Santiago), VII (Linares), VIII (Nuble) y XIV ${ }^{(\mathrm{N})}\left(\right.$ Valdivia $\left.^{(\mathrm{N})}\right)$.

Rhopalosiphum padi (Linnaeus, 1758).- Sobre Agrostis, Ag. verticillata, Arundo donax, Avena, Av. barbata, Av. fatua, Av. sativa, Bromus, B. berteroanus, B. catharticus, B. hordeaceus. B. rigidus, Capriola dactylon, Carex, Car. setifolia, Chusquea valdiviensis, Cyperus eragrostis, Dactylis glomerata, Deschampsia berteroana, Echinochloa crus-galli, Elymus angulatus, Eragrostis virescens, Gastridium, Hordeum, H. brachyantherum, H. chilense, H. murinum, H. vulgare, Juncus bufonius, Lolium perenne, Nassella chilensis, Oryza sativa, Paspalum dilatatum, Pa. distichum, Pa. notatum, Pa. vaginatum, Poa, Poa pratensis, Poaceae, Polypogon, Pol. australis, Pol. interruptus, Pol. linearis, Prunus, Secale cereale, Sisyrinchum graminifolium, Sorghum halepense, Triticum, T. aestivum y Zea mays. Las citas de Starý (1995) sobre una especie indeterminada de Brassicaceae, y de Zúñiga (1967b) sobre Cucumis sativus, Medicago sativa, Nicotiana tabacum, Phaseolus vulgaris y Solanum lycopersicon deben ser consideradas como accidentales. En las Regiones XV (Arica), I, II (El Loa ${ }^{(\mathrm{N})}$, Tocopilla $\left.{ }^{(\mathrm{N})}\right)$, III, IV, V (Isla de Pascua, Los Andes, Marga Marga, Petorca, Quillota, San Felipe de Aconcagua, Valparaíso), M (Cordillera, Santiago), VI (Cachapoal, Cochagua), VII (Curicó, Linares, Talca), VIII (Arauco, Bío Bío, Nuble ${ }^{(\mathbb{N})}$ ), IX (Cautín, Malleco), XIV (Valdivia), X (Llanquihue), XI y XII.

Rhopalosiphum rufiabdominale (Sasaki, 1889).- Sobre Avena sativa, Hordeum vulgare, Secale cereale, Solanum tuberosum, Triticum, T. aestivum. La cita de esta especie de Hille Ris Lambers (1975) sobre Berberis buxifolia debe ser considerada accidental. En las Regiones IV, V, M, VI, VII (Curicó(N) y VIII.

Schizaphis graminum (Rondani, 1852).- Sobre Agrostis, Ag. verticillata, Arundo donax, Avena, Av. barbata, Av. fatua, Av. sativa, Bromus, B. catharticus, Capriola dactylon, Elymus gayanus, Hordeum, H. murinum, H. vulgare, Lolium, L. perenne, Nassella chilensis, Paspalum dilatatum, Poaceae, Poa pratensis, Polypogon linearis, Secale cereale, Sisyrinchum graminifolium, Sorghum bicolor ${ }^{(\mathrm{N})}$, So. halepense, Stipa, Triticum, T. aestivum y Zea mays. En las Regiones I, II, III (Copiapó), IV, V (Los Andes, Petorca, Quillota, San Felipe de Aconcagua, Valparaíso), M (Cordillera, Santiago), VI (Cachapoal, Colchagua), VII (Curicó, Talca), VIII (Arauco), IX, XIV, X, XI y XII.

Sipha flava (Rondani, 1852).- Sobre Digitaria sanguinalis y Sorghum halepense. En las Regiones $\mathrm{V}^{(\mathrm{N})}\left(\right.$ Valparaíso $\left.^{(\mathrm{N})}\right)$ y $\mathrm{M}$ (Santiago).

Sipha (Rungsia) maydis Passerini, 1860.- Sobre Hordeum. En la Regiones IV (Elqui) y $\mathrm{V}^{(\mathrm{N})}\left(\operatorname{Los} \operatorname{Andes}^{(\mathrm{N})}\right)$.

Sitobion avenae (Fabricius, 1775).- Sobre Agrostis, Ag. capillaris, Ag. semiverticillata, Ag. verticillata, Arundinaria ${ }^{(\mathrm{N})}$, Avena, Av. barbata, Av. fatua, Av. sativa, Briza minor, Bromus, Bro. berteroanus, Bro. catharticus, Bro. hordeaceus, Bro. rigidus, Cynosurus echinatus, Dactylis, Da. glomerata, Deschampsia berteroana, Echinochloa crus-galli, Elymus, El. gayanus, El. repens, Eragrostis virescens, Hordeum, H. chilense, $H$. murinum, H. vulgare, Koeleria gerardii, Lolium, L. perenne, Melica striata, Nassella chilensis, Notholcus lanatus, Oryza sativa, Paspalum distichum, Pa. vaginatum, Phalaris amethystina, Ph. canariensis, Ph. minor, Poa, Poa pratensis, Poaceae, Polypogon, Pol. australis, Pol. interruptus, Pol. linearis, Pol. monspeliensis, Secale cereale, Setaria parviflora, Set. verticillata, Sorghum halepense, Stipa, Sisyrinchium, Triticum, T. aestivum y T. turgidum var. durum. En las Regiones III, IV, V (Los Andes, Marga Marga, Petorca, San Antonio, San Felipe de Aconcagua, Valparaíso), M (Cordillera, Maipo, 
Melipilla, Santiago, Talagante), VI (Cachapoal, Cardenal Caro, Colchagua), VII (Cauquenes, Curicó, Linares, Talca), VIII (Arauco, Bío Bío, Ñuble, Concepción), IX (Cautín, Malleco), XIV (Ranco $^{(\mathrm{N})}$, Valdivia), X (Chiloé $^{(\mathrm{N})}$, Llanquihue, Osorno, Palena $\left.^{(\mathrm{N})}\right), \mathrm{XI}^{(\mathrm{N})}\left(\right.$ Coihaique $\left.^{(\mathrm{N})}\right)$ y XII ${ }^{(\mathrm{N})}\left(\right.$ Magallanes $\left.^{(\mathrm{N})}\right)$.

Sitobion fragariae (Walker, 1848).- Sobre Avena barbata, A. sativa, Gladiolus, Hordeum, H. vulgare, Notholcus lanatus, Poaceae, Rubus y Triticum aestivum. Tomamos como accidental la cita de Starý (1995) de esta especie sobre Carpobrotus chilensis (Cactaceae). En las Regiones V (Los Andes, Quillota, San Felipe de Aconcagua, Valparaíso), M (Santiago), VI (Cachapoal), VII (Cauquenes, Talca), VIII (Bío Bío, Nuble), IX (Cautín, Malleco), XIV (Valdivia) y XI (Coihaique).

Smynthurodes betae Westwood, 1849.- Sobre Asparagus officinalis, Brassica napus, Chenopodium quinoa, Phaseolus vulgaris, Pisum sativum, Raphanus sativus, Solanum tuberosum y Vicia faba. En las Regiones I, V (Marga Marga), M, VI, VII, VIII, IX (Cautín $\left.{ }^{(\mathrm{N})}\right)$ y X (Llanquihue $\left.{ }^{(\mathrm{N})}\right)$.

Takecallis arundinariae (Essig, 1917).- Sobre bambúes (Poaceae) no identificados. En las Regiones $\mathrm{V}^{(\mathrm{N})}\left(\right.$ Valparaíso $\left.^{(\mathrm{N})}\right)$ y VI (Cachapoal).

Takecallis taiwana (Takahashi, 1926).- Sobre Phyllostachys bambusoides. En las Regiones $\mathrm{I}^{(\mathrm{N})}\left(\right.$ Iquique $\left.^{(\mathrm{N})}\right), \mathrm{II}^{(\mathrm{N})}\left(\mathrm{El} \mathrm{Loa}{ }^{(\mathrm{N})}\right)$, M (Santiago) y VII ${ }^{(\mathrm{N})}(\text { Talca })^{(\mathrm{N})}$.

Thecabius populimonilis (Riley, 1879).- Sobre Populus. En la Región V (Petorca).

Therioaphis (Pterocallidium) trifolii (Monell, 1882).- Citada siempre en Chile sin mención de subespecie; cabe la posibilidad de la presencia de dos de ellas a tenor de la presencia de ambas en América del Sur según Blackman \& Eastop (2016). Sobre Medicago sativa, Trifolium, T. pratense y T. repens. En las Regiones I (Iquique $\left.{ }^{(\mathrm{N})}\right), \mathrm{II}^{(\mathrm{N})}\left(\right.$ Antofagasta $\left.^{(\mathrm{N})}, \mathrm{El} \mathrm{Loa}^{(\mathrm{N})}\right), \mathrm{V}, \mathrm{M}$ $\left(\right.$ Maipo $\left.^{(\mathrm{N})}\right)$, VI, VII, VIII (Nuble $\left.{ }^{(\mathrm{N})}\right)$ y IX (Cautín).

Thripsaphis unciniae Quednau, 1990.- Sobre Carex y Uncinia. En las Regiones VIII (Bío Bío) y XIV (Valdivia).

Tinocallis saltans (Nevsky, 1929).- Sobre Ulmus. En la Región M $\left(\right.$ Santiago ${ }^{(\mathrm{N})}$, Maipo).

Tuberculatus (Tuberculoides) annulatus (Hartig, 1841).- Sobre Quercus. En las Regiones V(N) (Valparaíso $\left.^{(\mathrm{N})}\right), \mathrm{M}^{(\mathrm{N})}\left(\right.$ Santiago $\left.^{(\mathrm{N})}\right)$, $\mathrm{VI}^{(\mathrm{N})}\left(\right.$ Colchagua $\left.^{(\mathrm{N})}\right)$, VII ${ }^{(\mathrm{N})}\left(\right.$ Cauquenes $^{(\mathrm{N})}$, Curicó( $\left.{ }^{(\mathrm{N})}\right)$, VIII (Concepción $^{(\mathrm{N})}$, Ñuble), IX (Malleco) y XIV (Valdivia).

Tuberculatus querceus (Kaltenbach, 1843).- Sobre Quercus robur. En la Región M (Melipilla ${ }^{(\mathrm{N})}$, Santiago).

Tuberolachnus salignus (Kaltenbach, 1843).- Sobre Populus, Salix y S. viminalis. La mención de Melanoxanthus salici (sic), hoy Pterocomma salicis (Linnaeus, 1758), hecha por Capdeville (1945) se puede atribuir sin duda a T. salignus por los caracteres morfológicos que el autor expone. Olalquiaga Fauré (1946) citó esta especie sobre Malus domestica y Pyrus communis, indicando que quizás fuese accidental sobre esos árboles (Prado, 1991 y Klein Koch \& Waterhouse, 2000 recogen la mención, pero sin la aclaración). No obstante uno de los autores (O.) ha visto grupos de esta especie en frutales en la provincia argentina de Mendoza (dato no publicado). En las Regiones I, II (Antofagasta $\left.{ }^{(N)}\right)$, III, IV, V, M (Santiago), VI, VII, VIII, IX (Malleco), XIV, X, XI (General Carrera ${ }^{(\mathrm{N})}$ ) y XII.

Uroleucon (Uromelan) aeneum (Hille Ris Lambers, 1939).Sobre Carduus, Ca. albidus, Cirsium, Ci. vulgare $^{(\mathrm{N})}$ y Silybum marianum. En las Regiones V (Quillota, Valparaíso), VII ${ }^{(\mathrm{N})}$ Linares $\left.^{(\mathrm{N})}\right)$, VIII $^{(\mathrm{N})}\left(\right.$ Nuble $\left.^{(\mathrm{N})}\right)$ y IX $\left(\right.$ Cautín $^{(\mathrm{N})}$, Malleco).

Uroleucon ambrosiae lizerianum (Blanchard (E.E.), 1922).- Las citas de esta especie en Chile se han dado bajo los nombres U. ambrosiae (Thomas, 1878) — sin especificar subespecie-, U. lizerianum (Blanchard) y U. aaroni (Knowlton). Esta última especie es nomen dubium, aunque también podría ser considerada sinonimia de U. ambrosiae (Thomas) (de Carvalho et al., 1998; Blackman \& Eastop, 2016) y además su cita en Chile por Zúñiga (1967a) fue dada con duda; como U. ambrosiae es una especie muy común en toda América del Sur y también en Chile parece adecuado asignar a esta especie la cita de Zúñiga (1967a). De acuerdo con lo establecido por de Carvalho et al. (1998) y Nieto Nafría et al. (2007), U. ambrosiae está representada en América del Sur por U. ambrosiae lizerianum. Sobre Artemisia, Asteraceae, Baccharis y Lactuca sativa. En las Regiones XV (Arica), IV (Elqui), V (Marga Marga, Petorca, Valparaíso), M, VI, VII, VIII (Bío Bío, Ñuble), IX, X (Llanquihue, Osorno), XI (Aisén) y XII.

Uroleucon (Lambersius) bereticum (Blanchard (E.E.), 1922).Sobre Asteraceae, Baccharis y Conyza. En las Regiones VII (Curicó, Talca $\left.^{(\mathrm{N})}\right)$, VIII (Nuble), IX (Cautín ${ }^{(\mathrm{N})}$, Malleco) y X (Llanquihue, Osorno y Palena).

Uroleucon (Lambersius) brevisiphon de Carvalho, 1998.- Sobre Baccharis, B. linearis ${ }^{(\mathrm{N})}$, B. patagonica y dudosamente (de Carvalho et al., 1998) Grindelia. En las Regiones M (Santiago), VIII (Ñuble), IX (Cautín) y X (Llanquihue).

Uroleucon (Lambersius) chilense (Essig, 1953).- Sobre Asteraceae, Baccharis y B. salicifolia. En las Regiones IV (Elqui) y M (Santiago).

Uroleucon (Uromelan) compositae (Theobald, 1915).- Sobre Asteraceae, Cynara cardunculus. En las Regiones $\mathrm{V}^{(\mathrm{N})}$ (Los Andes $\left.^{(\mathrm{N})}\right)$, VIII (Ñuble) y IX (Malleco).

Uroleucon (Lambersius) erigeronense (Thomas, 1878).- Sobre Asteraceae, Baccharis y Haplopoppus. Consideramos como accidental la cita de Carvalho et al. (1998) de esta especie sobre Satureja gilliesii. En las Regiones V (Quillota), M (Santiago) y VII (Talca).

Uroleucon (Lambersius) essigi de Carvalho, 1998.- Sobre Baccharis. En la Región IV (Elqui).

Uroleucon (Lambersius) eumadiae Delfino \& Gonzales, 2005.Sobre Madia chilensis y M. sativa. En la Región M (Santiago).

Uroleucon (Lambersius) macolai (Blanchard (E.E.), 1922).Sobre Baccharis, B. linearis, B. salicifolia y Taraxacum officinale. En las Regiones IV (Elqui), V (Valparaíso), VII (Talca) y VIII (Bío Bío, Ñuble).

Uroleucon (Lambersius) muermosum (Essig, 1953).- Sobre Asteraceae y Senecio yegua. En las Regiones IX ${ }^{(\mathrm{N})}\left(\right.$ Cautín $\left.^{(\mathrm{N})}\right)$ y $\mathrm{X}$ (Llanquihue, Osorno).

Uroleucon (Lambersius) nuble (Essig, 1953).- Sobre Aster squamatus, Asteraceae y Senecio smithii. Las citas de Ramírez (2002) de esta especie sobre Acaena ovalifolia y Hebe salicifolia se pueden considerar accidentales. En las Regiones VIII (Ñuble) y XI (Coihaique)

Uroleucon (Lambersius) petrohuense de Carvalho, 1998.- Sobre Asteraceae. En la Región X (Llanquihue).

Uroleucon (Lambersius) pseudomuermosum de Carvalho, 1998.- Sobre Asteraceae, Baccharis y B. racemosa ${ }^{(\mathrm{N})}$. En las Regiones IX ${ }^{(\mathrm{N})}\left(\right.$ Cautín $\left.^{(\mathrm{N})}\right)$ y X (Llanquihue, Osorno).

Uroleucon sonchi (Linnaeus, 1767).- Sobre Hypochoeris radicata, Sonchus y S. oleraceus. En las Regiones V (Valparaíso, Quillota), $\mathrm{M}^{(\mathrm{N})}\left(\right.$ Santiago $\left.^{(\mathrm{N})}\right)$, VII (Talca), VIII (Ñuble) y X (Llanquihue).

Uroleucon (Lambersius) tessariae Delfino, 1994.- Sobre Pluchea absinthioides. En las Regiones XV (Arica), I (Iquique), IV (Choapa, Elqui), M (Santiago) y VI (Cachapoal).

Uroleucon (Lambersius) tucumani (Essig, 1953).- Sobre Baccharis. En las Regiones IV (Elqui) y $\mathrm{M}^{(\mathrm{N})}\left(\right.$ Cordillera $\left.^{(\mathrm{N})}\right)$.

Wahlgreniella nervata nervata (Gillette, 1908).- Citada en Chile sin especificar subespecie, pero la implicada tiene que ser la nominotípica. Los pulgones que Capdeville (1945) citó como «Aphis rosae» son inequívocamente de esta especie. Sobre Rosa. En las Regiones II ${ }^{(\mathrm{N})}\left(\mathrm{El} \mathrm{Loa}^{(\mathrm{N})}\right), \mathrm{M}$ (Santiago) y XI (General Carrera). 


\section{Especies no incluidas en el Catálogo}

En el Catálogo no se han incluido Phorodon humuli (Schrank, 1801) ni Thychea brevicornis Hart, 1894 citadas por Capdeville (1945), porque no hay concordancia entre las características, morfológicas o bionómicas que el autor proporcionó y las que realmente presentan esas especies, de las que no hay ninguna otra constancia de presencia en territorio chileno y que no constan en los catálogos de Smith \& Cermeli (1979), Fuentes-Contreras et al. (1997) y Anónimo (2010).

Tampoco se ha incluido en el catálogo Andinaphis paradoxa (Mier Durante, Ortego \& Nieto Nafría, 1996) porque su mención por Blackman \& Eastop (2006) se debió a una deficiente anotación (R. Blackman y P. Brown, com. pers.).

\section{Especies excluidas del Catálogo}

Aphis (Toxoptera) citricidus (Kirkaldy, 1907) no debe mantenerse en el catálogo chileno de pulgones como han demostrado Nieto Nafría et al. (2015).

Aphis medicaginis Koch, 1854, Aphis rumicis De Geer, 1773, Dysaphis apiifolia petroselini (Börner, 1950), Lipaphis erysimi (Kaltenbach, 1843) у Uroleucon aaroni (Knowlton, 1947) figuran en las listas de Smith \& Cermeli (1979), Fuentes-Contreras et al. (1997) y Anónimo (2010) pero no deben mantenerse en el Catálogo, por los motivos que se han aportado en las entradas de Aphis craccivora craccivora, Aphis fabae fabae, Dysaphis apiifolia apiifolia, Lipaphis pseudobrassicae y Uroleucon ambrosiae lizerianum, respectivamente.

\section{LAS PLANTAS HOSPEDADORAS DE LOS PULGONES EN CHILE}

Se han registrado en Chile 978 relaciones plantapulgón, entre las 169 especies o subespecies de pulgones relacionadas y sus 426 plantas hospedadoras, a cualquier nivel de identificación desde familia (Tabla 1). Entre esas relaciones se encuentran 189 que no están entre las recogidas por Blackman \& Eastop (2016), de las que unas 120 son escasamente relevantes por implicar bien a pulgones polífagos bien a pulgones que viven sobre géneros de muchas familias de plantas o sobre numerosos géneros de Poaceae, como Aphis (Toxoptera) aurantii, A. craccivora, A. fabae, A. gossypii, A. nerii, A. spiraecola, Aulacorthum solani, Brachycaudus helichrysi, Macrosiphum euphorbiae, Metopolophium dirhodum, Myzus ornatus, My. (Nectarosiphon) persicae, Rhopalosiphum padi, Schizaphis graminum o Sitobion avenae.

\section{Afidofaunas Regionales y provinciales de Chile}

La composición de la afidofauna de cada una de las regiones de Chile se muestra en la Tabla 2. El número de especies o subespecies registradas en cada una de las 15 regiones de Chile (Tabla 2) es muy variado, desde las 22 (13,02\% del total) de la más norteña Región XV o las 27 (15,98\%) de la más meridional Región XII, hasta las $85(50,30 \%)$ de la Región VII, $88(52,07 \%)$ de la VIII, las $92(54,44 \%)$ de la V y las $93(55,03 \%)$ de la Metropolitana.

Solamente Cinara cupressi, Macrosiphum euphorbiae, Myzus persicae persicae y Rhopalosiphum padi son conocidas en todas las regiones; todas ellas tienen una amplia distribución, C. cupressi está ligada a los cipreses, $R$. padi se desarrolla sobre multitud de gramíneas y las otras dos son polífagas. Otras seis especies están registradas en 14 regiones, Acyrthosiphon pisum, Brevicoryne brassicae, Cavariella aegopodii, Macrosiphum rosae, Schizaphis graminum y Tuberolachnus salignus, todas tienen distribuciones amplias o muy amplias y afectan a plantas silvestres, naturales o introducidas, y cultivadas con distintas finalidades. Por el contrario 38 especies $(22,49 \%$ del total) se han citado en menos de 2 regiones, $70(41,42 \%)$ en 2 a 5 regiones y $32(18,93 \%)$ en 6 a 10 regiones.

Las diferencias en el número de especies presentes en las parejas de regiones XV y I y XIV y X son muy patentes, respectivamente 22 y 37 en cada una de las de la primera pareja y 52 y 64 en cada una de las de la segunda pareja. La causa determinante de ambas es histórico-administrativa: las Regiones XV y XIV fueron establecidas 2007 a partir de las Regiones I y X respectivamente, que mantuvieron sus nombres. Las citas de pulgones sin localización provincial se han asignado solamente a una u otra (I o X). En el conjunto de las Regiones I y $\mathrm{XV}$ se han citado 43 especies (poco más de las 37 de la Región I), casi la misma cantidad que se han citado en las Regiones III y XI y más que en la Región II. En el conjunto de las Regiones XIV y X se han registrado 84 especies, cantidad que es cercana a la registrada en las regiones VII y VIII y superior a la de la Región IX.

Orillando lo anterior, las diferencias interregionales se deben fundamentalmente, sin duda, a motivos climáticos y de vegetación y secundariamente a la intensidad de las prospecciones. Las características climáticas del Norte Grande (en el que se encuentran las Regiones XV, I y II) y del Chile Austral (en el que se encuentran las Regiones XI y XII) son menos propicias que las existentes en el Chile Central (Regiones V, M, VI, VII y VIII) para el desarrollo de la mayor parte de las especies de pulgones presentes en Chile, aunque las especies autóctonas son propias de zonas con clima húmedo y templado o frío, como evidencia su presencia en las Regiones meridionales.

La bibliografía muestra que la investigación afídica en Chile ha estado focalizada especialmente en las regiones IV a $\mathrm{X}$, ambas inclusive, y sobre plantas cultivadas, situación que se pone de manifiesto en las grandes diferencias de especies registradas en provincias de la misma Región (Tabla 3).

Las diferencias en la cantidad de especies registradas en provincias de la misma Región (Tabla 3) 
Tabla 1.- Plantas hospedadoras de los pulgones citados en Chile. Las relaciones pulgón-planta que no figuran en Aphids on World's Plants (Blackman \& Eastop, 2016) se señalan con la abreviatura "n.r." (nueva relación) entre corchetes.

Table 1.- Host plants of aphids recorded from Chile. Abbreviation "n.r." (new relationship) into brackets announce the aphid-host plant relationships that are not listed in Aphids on World's Plants (Blackman \& Eastop, 2016).

Acacia (Fabaceae)

Aphis craccivora craccivora

Aphis spiraecola

Acanthus mollis (Acanthaceae)

Hyperomyzus lactucae [n.r.]

Macrosiphum euphorbiae

Acca sellowiana (Myrtaceae)

Macrosiphum euphorbiae [n.r.]

Myzus (N.) persicae persicae [n.r.]

Acer (Aceraceae)

Drepanosiphum oregonense

Acer pseudoplatanus

Drepanosiphum oregonense

Actinidia chinensis var. deliciosa (Actinidiaceae)

Aphis spiraecola [n.r.]

Macrosiphum euphorbiae [n.r.]

Myzus (N.) persicae persicae [n.r.]

Adesmia (Asteraceae)

Aphis craccivora craccivora

Agrostis (Poaceae)

Rhopalosiphum padi

Schizaphis graminum

Sitobion avenae

Agrostis capillaris

Geoica lucifuga [n.r.]

Sitobion avenae

Agrostis semiverticillata

Sitobion avenae [n.r.]

Agrostis verticillata

Metopolophium dirhodum [n.r.]

Rhopalosiphum padi [n.r.]

Schizaphis graminum [n.r.]

Sitobion avenae [n.r.]

Alisma (Alismataceae)

Rhopalosiphum nymphaeae

Allium cepa (Amaryllidaceae)

Aphis gossypii

Neotoxoptera formosana

Allium porrum

Neotoxoptera formosana

Allium schoenoprasum

Neotoxoptera oliveri

Alnus cordata (Betulaceae)

Pterocallis alni

Alnus glutinosa

Pterocallis alni

Alstroemeria (Alstroemeriaceae)

Aphis alstroemeriae

Alstroemeria leporina

Macrosiphum euphorbiae [n.r.]

Amsinckia hispida (Boraginaceae)

Brachycaudus helichrysi [n.r.]

Amygdalus communis (Rosaceae)

Brachycaudus helichrysi

Brachycaudus (S.) persicae

Myzus (N.) persicae persicae

Anethum graveolens (Apiaceae)

Cavariella aegopodii

Macrosiphum euphorbiae [n.r.]

Annona cherimola (Annonaceae)

Aphis gossypii

Aphis spiraecola

Anoda (Malvaceae)
Myzus (N.) persicae persicae [n.r.]

Apiaceae

Aulacorthum solani solani

Cavariella aegopodii

Hyadaphis passerinii

Apium graveolens (Apiaceae)

Cavariella aegopodii

Dysaphis apiifolia apiifolia

Dysaphis foeniculus

Apium australe var. angustisectum

Cavariella aegopodii [n.r.]

Aralia (Araliaceae)

Aphis hederae

Arrhenatherum elatius (Poaceae)

Geoica lucifuga [n.r.]

Artemisia (Asteraceae)

Aphis craccivora craccivora

Uroleucon ambrosiae lizerianum

Arundinaria (Poaceae)

Sitobion avenae [n.r.]

Arundo donax (Poaceae)

Melanaphis donacis

Rhopalosiphum maidis

Rhopalosiphum padi

Schizaphis graminum

Asparagus (Asparagaceae)

Aulacorthum solani solani

Asparagus officinalis

Aphis gossypii

Macrosiphum euphorbiae

Myzus (N.) persicae persicae

Smynthurodes betae [n.r.]

Aster squamatus (Asteraceae)

Uroleucon (L.) nuble [n.r.]

Asteraceae

Aphis craccivora craccivora

Macrosiphoniella tanacetaria bonariensis

Myzus (N.) persicae persicae

Nasonovia ribisnigri

Uroleucon ambrosiae lizerianum

Uroleucon (L.) bereticum

Uroleucon (Lambersius) chilense

Uroleucon (U'an.) compositae

Uroleucon (L.) erigeronense

Uroleucon (L.) muermosum

Uroleucon (L.) nuble

Uroleucon (L.) petrohuense

Uroleucon (L.) pseudomuermosum

Astragalus germaini (Fabaceae)

Acyrthosiphon pisum

Austrocedrus chilensis (Cupressaceae)

Cinara (Cu.) cupressi [n.r.]

Cinara (Cu.) cupressivora [n.r.]

Cinara (Cu.) fresai [n.r.]

Cinara (Cu.) tujafilina [n.r.]

Avena (Poaceae)

Metopolophium dirhodum

Rhopalosiphum maidis

Rhopalosiphum padi

Schizaphis graminum

Sitobion avenae

Avena barbata

Rhopalosiphum padi [n.r.] 
Tabla 1.- (Continuación)

\begin{tabular}{|c|c|}
\hline Schizaphis graminum & Brevicoryne brassicae \\
\hline Sitobion avenae & Lipaphis pseudobrassicae \\
\hline Sitobion fragariae & Myzus (N.) persicae persicae \\
\hline Avena fatua & Smynthurodes betae \\
\hline Metopolophium dirhodum & Brassica nigra \\
\hline Myzus (N.) persicae persicae & Brevicoryne brassicae \\
\hline Rhopalosiphum padi & Brassica oleracea \\
\hline Schizaphis graminum & Aphis gossypii [n.r.] \\
\hline Sitobion avenae & Brevicoryne brassicae \\
\hline Avena sativa & Myzus (N.) persicae persicae \\
\hline Diuraphis noxia & Brassica oleracea var. botrytis \\
\hline Metopolophium dirhodum & Aphis gossypii \\
\hline Metopolophium festucae cerealium & Brevicoryne brassicae \\
\hline Rhopalosiphum maidis & Myzus (N.) persicae persicae \\
\hline Rhopalosiphum padi & Brassica oleracea var. capitata \\
\hline Rhopalosiphum rufiabdominale & Aphis craccivora \\
\hline Schizaphis graminum & Aphis gossypii \\
\hline Sitobion avenae & Brevicoryne brassicae \\
\hline Sitobion fragariae & Macosiphum euphorbiae \\
\hline Baccharis (Asteraceae) & Myzus (N.) persicae persicae \\
\hline Aphis craccivora craccivora [n.r.] & Brassica oleracea var. gemmifera \\
\hline Uroleucon ambrosiae lizerianum & Brevicoryne brassicae \\
\hline Uroleucon (L.) bereticum & Myzus (N.) persicae persicae \\
\hline Uroleucon (Lambersius) brevisiphon & Brassica oleracea var. italica \\
\hline Uroleucon (Lambersius) chilense & Brevicoryne brassicae \\
\hline Uroleucon (L.) erigeronense & Brassica oleracea var. viridis \\
\hline Uroleucon (L.) essigi & Aphis craccivora craccivora \\
\hline Uroleucon (L.) macolai & Aphis gossypii \\
\hline Uroleucon (L.) pseudomuermosum [n.r.] & Brevicoryne brassicae \\
\hline Uroleucon (L.) tucumani & Macrosiphum euphorbiae \\
\hline Baccharis linearis & Myzus (N.) persicae persicae \\
\hline Brachycaudus helichrysi [n.r.] & Brassica rapa \\
\hline Myzus (N.) persicae persicae [n.r.] & Brevicoryne brassicae \\
\hline Uroleucon (Lambersius) brevisiphon [n.r.] & Myzus (N.) persicae persicae \\
\hline Uroleucon (L.) macolai & Briza minor (Poaceae) \\
\hline Baccharis patagonica & Sitobion avenae [n.r.] \\
\hline Uroleucon (Lambersius) brevisiphon & Bromus (Poaceae) \\
\hline Baccharis racemosa & Metopolophium dirhodum \\
\hline Uroleucon (L.) pseudomuermosum [n.r.] & Myzus (N.) persicae persicae \\
\hline Baccharis salicifolia & Rhopalosiphum padi \\
\hline Aphis craccivora craccivora [n.r.] & Schizaphis graminum \\
\hline Uroleucon (Lambersius) chilense [n.r.] & Sitobion avenae \\
\hline Uroleucon (L.) macolai & Bromus berteroanus \\
\hline Bahia ambrosioides (Asteraceae) & Metopolophium dirhodum [n.r.] \\
\hline Aphis spiraecola & Rhopalosiphum padi [n.r.] \\
\hline Berberis darwini (Berberidaceae) & Sitobion avenae [n.r.] \\
\hline Aphis berberidorum & Bromus catharticus \\
\hline Berberis microphylla & Metopolophium dirhodum \\
\hline Aphis patagonica & Rhopalosiphum padi \\
\hline Beta vulgaris (Amaranthaceae) & Schizaphis graminum \\
\hline Aphis fabae fabae & Sitobion avenae \\
\hline Brevicoryne brassicae & Bromus hordeaceus \\
\hline Macrosiphum euphorbiae & Metopolophium dirhodum \\
\hline Myzus (N.) persicae persicae & Rhopalosiphum padi \\
\hline Beta vulgaris subsp. cicla & Sitobion avenae \\
\hline Aphis fabae fabae & Bromus rigidus \\
\hline Myzus (N.) persicae persicae & Metopolophium dirhodum [n.r.] \\
\hline Bidens aurea (Asteraceae) & Rhopalosiphum padi \\
\hline Aphis spiraecola [n.r.] & Sitobion avenae \\
\hline Myzus ornatus [n.r.] & Cactaceae \\
\hline Bignonia (Bignoniaceae) & Aphis craccivora craccivora \\
\hline Aphis gossypii [n.r.] & Caesalpinia angulata (Fabaceae) \\
\hline Myzus ornatus [n.r.] & Acyrthosiphon kondoi [n.r.] \\
\hline Brassica (Brassicaceae) & Aphis spiraecola [n.r.] \\
\hline Brevicoryne brassicae & Calandrinia parviflora (Montiaceae) \\
\hline Myzus (N.) persicae persicae & Aphis spiraecola \\
\hline Brassica napus & Calceolaria (Calceolariaceae) \\
\hline
\end{tabular}


Tabla 1.- (Continuación)

\begin{tabular}{|c|c|}
\hline Aphis spiraecola & Chenopodium quinoa \\
\hline Calendula officinalis (Astreceae) & Smynthurodes betae [n.r.] \\
\hline Myzus (N.) persicae persicae & Chrysanthemum (Asteraceae) \\
\hline Callitropsis macrocarpa (Cupressaceae) & Aphis fabae fabae \\
\hline Cinara (Cu.) cupressi & Aulacorthum solani \\
\hline Cinara $(\mathrm{Cu}$.$) fresai$ & Brachycaudus helichrysi \\
\hline Canna (Cannaceae) & Macrosiphoniella sanborni \\
\hline Macrosiphum euphorbiae & Myzus (N.) persicae persicae \\
\hline Capriola dactylon (Poaceae) & Pleotrichophorus chrysanthemi \\
\hline Metopolophium dirhodum & Chrysanthemum coronarium \\
\hline Rhopalosiphum padi & Aphis fabae fabae \\
\hline Schizaphis graminum & Myzus (N.) persicae persicae \\
\hline Capsella bursa-pastoris (Brassicaceae) & Chrysanthemum morifolium \\
\hline Aphis gossypii & Brachycaudus helichrysi \\
\hline Myzus (N.) persicae persicae & Macrosiphoniella sanborni \\
\hline Capsicum annuum (Solanaceae) & Myzus (N.) persicae persicae \\
\hline Macrosiphum euphorbiae & Chusquea (Poaceae) \\
\hline Myzus (N.) persicae persicae & Aphis craccivora craccivora [n.r.] \\
\hline Capsicum frutescens & Aploneura lentisci [n.r.] \\
\hline Macrosiphum euphorbiae & Melanaphis donacis [n.r.] \\
\hline Myzus (N.) persicae persicae & Chusquea valdiviensis \\
\hline Carduus (Asteraceae) & Rhopalosiphum padi \\
\hline Uroleucon (U'an.) aeneum & Cichorium intybus (Asteraceae) \\
\hline Carduus albidus & Myzus ornatus \\
\hline Uroleucon (U'an.) aeneum & Nasonovia ribisnigri \\
\hline Carex (Cyperaceae) & Cirsium (Asteraceae) \\
\hline Rhopalosiphum padi & Aphis fabae fabae \\
\hline Thripsaphis unciniae [n.r.] & Uroleucon (U'an.) aeneum \\
\hline Carex setifolia & Cirsium vulgare \\
\hline Rhopalosiphum padi [n.r.] & Uroleucon (U'an.) aeneum \\
\hline Carica (Caricaceae) & Citrullus lanatus (Cucurbitaceae) \\
\hline Myzus ornatus [n.r.] & Aphis craccivora craccivora \\
\hline Carica papaya & Aphis gossypii \\
\hline Myzus ornatus [n.r.] & Citrus (Rutaceae) \\
\hline Carica pubescens & Aphis (T.) aurantii \\
\hline Myzus (N.) persicae persicae [n.r.] & Aphis fabae fabae \\
\hline Carpobrotus chilensis (Aizoaceae) & Aphis gossypii \\
\hline Aphis fabae fabae [n.r.] & Aphis spiraecola \\
\hline Myzus (N.) persicae persicae & Macrosiphum euphorbiae \\
\hline Carum (Apiaceae) & Myzus (N.) persicae persicae \\
\hline Hyadaphis foeniculi & Citrus aurantiifolia \\
\hline Cassia (Fabaceae) & Aphis spiraecola \\
\hline Aphis craccivora craccivora & Citrus limon \\
\hline Castanea sativa (Fagaceae) & Aphis (T.) aurantii \\
\hline Myzocallis (A.) castanicola castanicola & Aphis gossypii \\
\hline Cedrus (Pinaceae) & Aphis spiraecola \\
\hline Cinara cedri & Aulacorthum solani solani \\
\hline Cedrus libani & Macrosiphum euphorbiae \\
\hline Cinara cedri & Myzus (N.) persicae persicae \\
\hline Cerasus avium (Rosaceae) & Citrus maxima \\
\hline Myzus (N.) persicae persicae & Aphis $(T$.$) aurantii$ \\
\hline Cereus (Cactaceae) & Aphis craccivora craccivora \\
\hline Aphis craccivora craccivora & Aphis gossypii \\
\hline Macrosiphum euphorbiae & Aphis spiraecola \\
\hline Myzus (N.) persicae persicae & Aulacorthum solani solani [n.r.] \\
\hline Myzus ornatus & Macrosiphum euphorbiae [n.r.] \\
\hline Cestrum (Solanaceae) & Myzus (N.) persicae persicae [n.r.] \\
\hline Aphis fabae fabae & Citrus reticulata \\
\hline Myzus (N.) persicae persicae & Aphis (T.) aurantii \\
\hline Cestrum parqui & Aphis spiraecola \\
\hline Aphis fabae fabae & Aulacorthum solani solani \\
\hline Chamaecyparis (Cupressaceae) & Citrus $\mathrm{x}$ sinensis \\
\hline Cinara $(\mathrm{Cu}$.$) cupressi$ & Aphis craccivora craccivora \\
\hline Chenopodium (Chenopodiaceae) & Colletia (Rhamnaceae) \\
\hline Macrosiphum euphorbiae & Aphis conflicta \\
\hline Chenopodium album & Colletia spinosissima \\
\hline Myzus (N.) persicae persicae & Aphis conflicta \\
\hline
\end{tabular}


Tabla 1.- (Continuación)

Conium maculatum (Apiaceae)

Dysaphis apiifolia apiifolia

Convolvulus (Convolvulaceae)

Macrosiphum euphorbiae

Myzus (N.) persicae persicae

Convolvulus arvensis

Myzus (N.) persicae persicae

Conyza (Asteraceae)

Brachycaudus helichrysi

Uroleucon (L.) bereticum

Corylus avellana (Betulaceae) Myzocallis coryli

Cotoneaster (Rosaceae) Aphis spiraecola

Crataegus (Rosaceae) Aphis spiraecola

Cristaria (Malvaceae) Macrosiphum euphorbiae

Cucumis melo (Cucurbitaceae) Aphis gossypii Myzus (N.) persicae persicae

Cucumis sativus Macrosiphum euphorbiae

Cucurbita ficifolia (Cucurbitaceae) Aphis craccivora craccivora Aphis fabae fabae [n.r.] Aphis gossypii Aulacorthum solani solani [n.r.] Macrosiphum euphorbiae [n.r.] Myzus ornatus [n.r.] Myzus (N.) persicae persicae [n.r.]

Cucurbita maxima

Aphis craccivora craccivora Aphis gossypii

Aulacorthum solani solani [n.r.]

Macrosiphum euphorbiae

Myzus (N.) persicae persicae

Cupressaceae Cinara (Cu.) cupressi

Cupressus (Cupressaceae) Cinara (Cu.) cupressi Cinara (Cu.) fresai Cinara (Cu.) tujafilina Illinoia morrisoni

Cupressus macrocarpa Cinara $(\mathrm{Cu}$.$) fresai$

Cupressus sempervirens Cinara (Cu.) cupressi

Cydonia oblonga (Rosaceae) Aphis spiraecola Eriosoma (M.) pyricola Ovatus crataegarius

Cynara (Asteraceae) Myzus (N.) persicae persicae

Cynara cardunculus

Aphis craccivora craccivora Aphis fabae fabae

Aphis gossypii Aulacorthum solani solani Capitophorus elaeagni Dysaphis lappae cynarae Macrosiphum euphorbiae Uroleucon (U'an) compositae

Cynodon dactylon: véase Capriola dactylon

Cynosurus echinatus (Poaceae) Sitobion avenae

Cyperus eragrostis (Cyperaceae)
Rhopalosiphum padi

Dactylis (Poaceae)

Metopolophium dirhodum

Sitobion avenae

Dactylis glomerata

Metopolophium dirhodum

Rhopalosiphim padi

Sitobion avenae

Datura (Solanaceae)

Myzus (N.) persicae persicae

Datura stramonium Myzus (N.) persicae persicae

Daucus carota (Apiaceae)

Aphis gossypii

Cavariella aegopodii

Dysaphis foeniculus Hyadaphis passerinii

Deschampsia berteroana (Poaceae)

Rhopalosiphum padi [n.r.]

Sitobion avenae [n.r.]

Dianthus (Caryophyllaceae) Myzus (N.) persicae persicae

Digitaria sanguinalis (Poaceae) Sipha flava

Discaria chacaye (Rhamnaceae) Aphis conflicta

Drimys winteri (Winteraceae) Aphis nasturtii [n.r.]

Echinochloa (Poaceae) Rhopalosiphum maidis

Echinochloa crus-galli Rhopalosiphum maidis Rhopalosiphum padi Sitobion avenae

Echinopsis chiloensis (Cactaceae) Aphis danielae

Elymus (Poaceae) Sitobion avenae

Elymus angulatus Metopolophium dirhodum [n.r.] Rhopalosiphum padi [n.r.]

Elymus gayanus Schizaphis graminum [n.r.] Sitobion avenae [n.r.]

Elymus repens Sitobion avenae

Epiphyllum (Cactaceae) Aphis fabae fabae [n.r.]

Eragrostis virescens (Poaceae) Rhopalosiphum padi [n.r.] Sitobion avenae [n.r.]

Eriobotrya japonica (Rosaceae) Aphis gossypii Aphis spiraecola Macrosiphum euphorbiae [n.r.]

Erodium cicutarium (Geraniaceae) Myzus (N.) persicae persicae

Eucalyptus (Myrtaceae) Aphis craccivora craccivora

Euphorbia (Euphorbiaceae) Aphis maulensis

Euphorbia peplus Myzus (N.) persicae persicae

Euphorbia pulcherrima Myzus (N.) persicae persicae

Fabaceae

Aphis craccivora craccivora 
Tabla 1.- (Continuación)

\section{Festuca (Poaceae)}

Metopolophium dirhodum

Fitzroya cupressoides (Cupressaceae)

Cinara (Cu.) cupressi [n.r.]

Foeniculum vulgare (Apiaceae)

Aphis fabae fabae

Cavariella aegopodii

Dysaphis apiifolia apiifolia

Hyadaphis passerinii

Fragaria (Rosaceae)

Aphis gossypii

Chaetosiphon $(P$.$) fragaefolii$

Chaetosiphon (P.) thomasi

Fragaria chiloensis (Rosaceae) Chaetosiphon (P.) fragefolii [n.r.]

Chaetosiphon (P.) thomasi [n.r.]

Fragaria $\mathrm{x}$ ananassa

Aphis forbesi

Chaetosiphon (P.) fragaefoli

Chaetosiphon (P.) thomasi

Rhodobium porosum

Fraxinus (Oleaceae)

Prociphilus fraxinifolii

Fuchsia magellanica (Onagraceae) Myzus ornatus

Fumaria officinalis (Papaveraceae) Macrosiphum euphorbiae

Gamochaeta spiciforme (Poaceae) Macrosiphum euphorbiae [n.r.]

Gardenia (Rubiaceae)

Brachycaudus helichrysii

Gastridium (Poaceae)

Rhopalosiphum padi [n.r.]

Geoffroea decorticans (Fabaceae)

Aphis craccivora craccivora

Geranium (Malvaceae)

Acyrthosiphon malvae malvae

Aulacorthum solani solani

Gerbera (Asteraceae)

Aphis gossypii

Gladiolus (Iridaceae)

Aphis fabae fabae

Myzus ornatus [n.r.]

Sitobion fragariae

Glycine max (Fabaceae)

Acyrthosiphon pisum

Aphis gossypii

Myzus ornatus

Neomyzus circumflexum

Gossypium (Malvaceae)

Aphis gossypii

Gossypium hirsutum

Aphis gossypii

Grindelia (Asteraceae)

? Uroleucon (Lambersius) brevisiphon [n.r.]

Gunnera (Gunneraceae)

Neuquenaphis valdiviana

Gunnera magellanica

Aphis carrilloi

Myzus ornatus [n.r.]

Neuquenaphis valdiviana

Gunnera tinctoria

Neuquenaphis valdiviana

Haplopoppus (Poaceae)

Uroleucon (L.) erigeronense

Hebe salicifolia (Plantaginaceae)

Myzus ornatus [n.r.]
Hedera helix (Araliaceae)

Aphis hederae

Helianthus annuus (Asteraceae)

Aphis asclepiadis

Aphis craccivora craccivora

Aphis fabae fabae

Aphis gossypii

Brachycaudus helichrysi

Macrosiphum euphorbiae

Myzus ornatus

Myzus (N.) persicae persicae

Helminthotheca echioides (Asteraceae)

Brachycaudus helichrysi [n.r.]

Macrosiphum euphorbiae [n.r.]

Hibiscus (Malvaceae)

Myzus (N.) persicae persicae

Hieracium (Asteraceae)

Nasonovia ribisnigri

Hirschfeldia incana (Brassicaceae)

Brevicoryne brassicae

Lipaphis pseudobrassicae [n.r.]

Myzus (N.) persicae persicae

Holcus lanatus: véase Notholcus lanatus

Hordeum (Poaceae)

Metopolophium dirhodum

Rhopalosiphum padi

Schizaphis graminum

Sipha (R.) maydis

Sitobion avenae

Sitobion fragariae

Hordeum brachyantherum (Poaceae)

Rhopalosiphon padi [n.r.]

Hordeum chilense

Metopolophium dirhodum [n.r.]

Rhopalosiphum padi [n.r.]

Sitobion avenae [n.r.]

Hordeum murinum

Metopolophium dirhodum

Myzus (N.) persicae persicae [n.r.]

Rhopalosiphum padi

Schizaphis graminum

Sitobion avenae

Hordeum vulgare

Diuraphis noxia

Metopolophium dirhodum

Metopolophium festucae cerealium

Myzus (N.) persicae persicae

Rhopalosiphum maidis

Rhopalosiphum padi

Rhopalosiphum rufiabdominale

Schizaphis graminum

Sitobion avenae

Sitobion fragariae

Hoya carnosa (Apocinaceae)

Aphis nerii

Hypochoeris radicata (Asteraceae)

Uroleucon sonchi

Ipomea batatas (Convovulaceae)

Myzus (N.) persicae persicae

Iris (Iridaceae)

Dysaphis tulipae

Macrosiphum euphorbiae

Iris germanica

Dysaphis tulipae

Jacaranda (Bignoniaceae)

Aphis gossypii

Jasminum (Oleaceae) 
Tabla 1.- (Continuación)

\begin{tabular}{|c|c|}
\hline Aphis spiraecola & Madia sativa \\
\hline Juglans regia (Juglandaceae) & Uroleucon (L.) eumadiae \\
\hline Chromaphis juglandicola & Magnolia grandiflora (Magnoliaceae) \\
\hline Myzus (N.) persicae persicae & Aphis spiraecola [n.r.] \\
\hline Juncus bufonius (Juncaceae) & Malus (Rosaceae) \\
\hline Rhopalosiphum padi & Aphis spiraecola \\
\hline Koeleria gerardii (Poaceae) & Myzus ornatus [n.r.] \\
\hline Sitobion avenae & Ovatus crataegarius \\
\hline Lactuca (Asteraceae) & Malus domestica \\
\hline Nasonovia ribisnigri & Aphis craccivora craccivora \\
\hline Pemphigus bursarius & Aphis gossypii \\
\hline Lactuca sativa & Aphis spiraecola \\
\hline Aulacorthum solani solani & Eriosoma lanigerum \\
\hline Hyperomyzus lactucae & Myzus ornatus [n.r.] \\
\hline Macrosiphum euphorbiae & Ovatus crataegarius \\
\hline Myzus ornatus & Malva (Malvaceae) \\
\hline Myzus (N.) persicae persicae & Acyrthosiphon malvae malvae \\
\hline Nasonovia ribisnigri & Myzus (N.) persicae persicae \\
\hline Pemphigus bursarius & Myzus ornatus \\
\hline Uroleucon ambrosiae lizerianum & Malva nicaensis \\
\hline Lamiaceae & Myzus (N.) persicae persicae [n.r.] \\
\hline Aphis gossypii & Mangifera indica (Anacardiaceae) \\
\hline Myzus (N.) persicae persicae & Aphis spiraecola \\
\hline Lathyrus latifolius (Fabaceae) & Marrubium vulgare (Lamiaceae) \\
\hline Acyrthosiphon pisum & Cryptomyzus ballotae \\
\hline Lathyrus magellanicus & Matricaria (Asteraceae) \\
\hline Myzus ornatus [n.r.] & Brachycaudus helichrysi \\
\hline Lathyrus sativus & Matthiola incana (Brassicaceae) \\
\hline Macrosiphum euphorbiae & Lipaphis pseudobrassicae [n.r.] \\
\hline Lathyrus subandinus & Maytenus boaria (Celastraceae) \\
\hline Acyrthosiphon pisum [n.r.] & Aphis spiraecola [n.r.] \\
\hline Lens culinaris (Fabaceae) & Medicago (Fabaceae) \\
\hline Acyrthosiphon kondoi & Acyrthosiphon pisum \\
\hline Acyrthosiphon pisum & Aphis craccivora craccivora \\
\hline Aphis craccivora craccivora & Medicago arabica \\
\hline Ligustrum (Oleaceae) & Acyrthosiphon pisum \\
\hline Aphis fabae fabae & Medicago sativa \\
\hline Myzus (N.) persicae persicae & Acyrthosiphon kondoi \\
\hline Lilium (Liliaceae) & Acyrthosiphon pisum \\
\hline Dysaphis tulipae & Aphis craccivora craccivora \\
\hline Lolium (Poaceae) & Aphis fabae fabae \\
\hline Aploneura lentisci [n.r.] & Aphis gossypii \\
\hline Geoica lucifuga [n.r.] & Aulacorthum solani solani [n.r.] \\
\hline Metopolophium dirhodum & Brachycaudus helichrysi \\
\hline Schizaphis graminum & Myzus ornatus \\
\hline Sitobion avenae & Myzus (N.) persicae persicae \\
\hline Lolium perenne & Nearctaphis bakeri \\
\hline Metopolophium dirhodum & Therioaphis $(P-)$ trifolii \\
\hline Rhopalosiphum padi & Melica striata (Poaceae) \\
\hline Schizaphis graminum & Sitobion avenae [n.r.] \\
\hline Sitobion avenae & Melilotus (Fabaceae) \\
\hline Lonicera (Caprifoliaceae) & Acyrthosiphon kondoi \\
\hline Hyadaphis passerinii & Melilotus albus \\
\hline Lotus (Fabaceae) & Acyrthosiphon kondoi \\
\hline Acyrthosiphon pisum & Melilotus indica \\
\hline Aphis craccivora craccivora & Acyrthosiphon kondoi \\
\hline Geoica lucifuga [n.r.] & Acyrthosiphon pisum \\
\hline Lupinus (Fabaceae) & Aphis craccivora craccivora \\
\hline Acyrthosiphon kondoi & Mulinum spinosum (Apiaceae) \\
\hline Acyrthosiphon pisum & Aphis roberti \\
\hline Brachycaudus helichrysi & Musa x paradisiaca (Musaceae) \\
\hline Lycium stenophyllum (Solanaceae) & Pentalonia nigronervosa \\
\hline Aphis danielae & Mutisia subulata (Asteraceae) \\
\hline Macadamia (Proteaceae) & Aphis craccivora craccivora \\
\hline Aphis spiraecola [n.r.] & Nassella chilensis (Poaceae) \\
\hline Madia chilensis (Asteraceae) & Rhopalosiphum padi [n.r.] \\
\hline Uroleucon (L.) eumadiae [n.r.] & Schizaphis graminum [n.r.] \\
\hline
\end{tabular}


Tabla 1.- (Continuación)

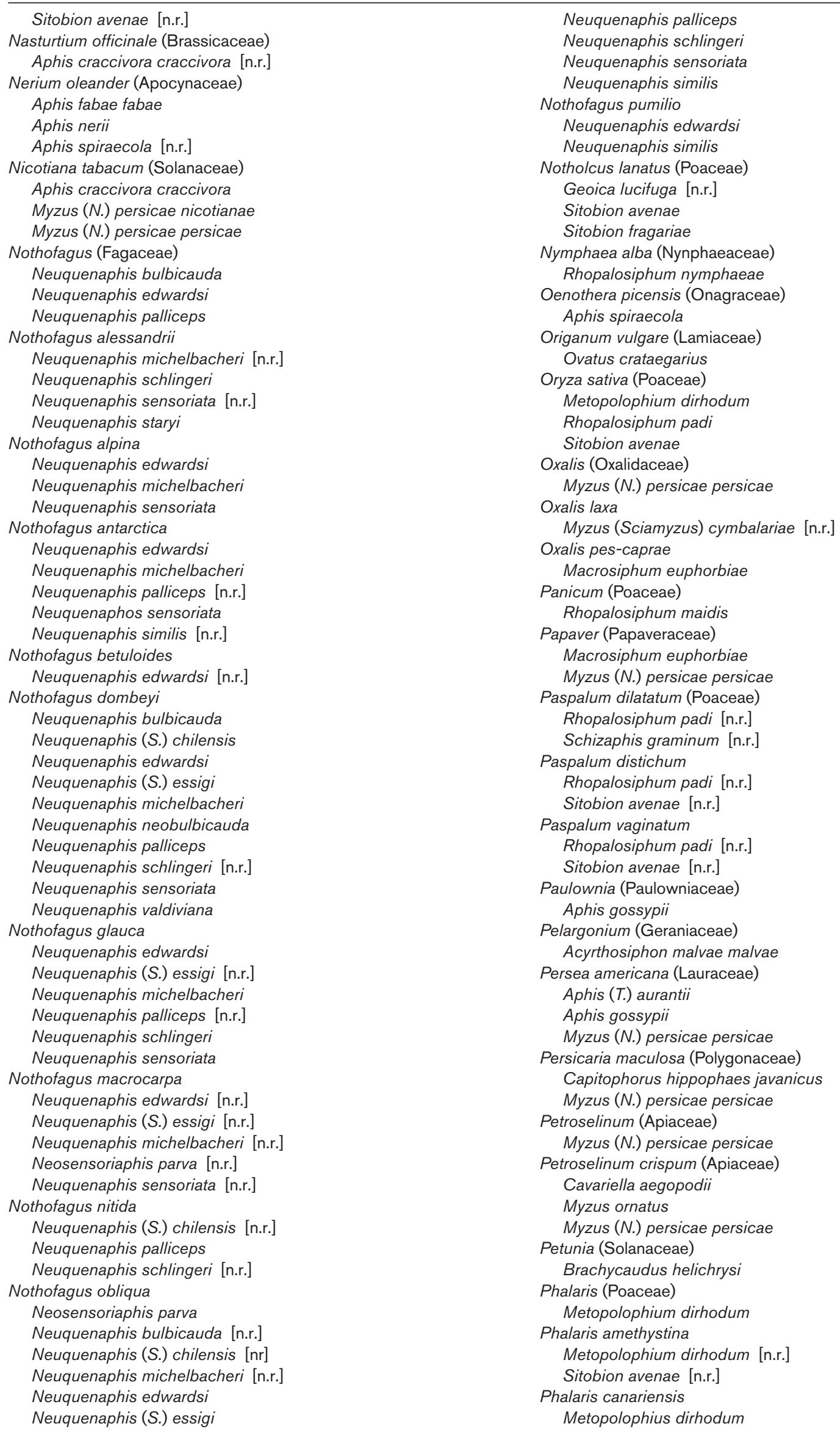


Tabla 1.- (Continuación)

\author{
Sitobion avenae [n.r.] \\ Phalaris minor \\ Metopolophium dirhodum [n.r.] \\ Sitobion avenae [n.r.] \\ Phaseolus (Fabaceae) \\ Macrosiphum euphorbiae \\ Phaseolus vulgaris \\ Aphis craccivora craccivora \\ Aphis fabae fabae \\ Aphis gossypii \\ Aulacorthum solani solani \\ Macrosiphum euphorbiae \\ Myzus (N.) persicae persicae \\ Rhopalosiphoninus latysiphon \\ Smynthurodes betae \\ Phrodus microphyllus (Solanaceae) \\ Eucarazzia elegans [n.r.] \\ Phyllostachys bambusoides (Poaceae) \\ Takecallis taiwana \\ Picea (Pinaceae) \\ Cinara pilicornis \\ Elatobium abietinum \\ Pilgerodendron uviferum (Cupressaceae) \\ Neophyllaphis (C.) michelbacheri
}

Picris echioides: véase Helmintotheca echioides

Pinus (Pinaceae)

Essigella californica

Eulachnus rileyi

Pinus contorta

Eulachnus rileyi

Pinus palustris

Cinara pinimaritimae [n.r.]

Pinus ponderosa

Eulachnus rileyi

Pinus radiata

Cinara pinimaritimae

Essigella californica

Eulachnus rileyi

Pistacia vera (Anacardiaceae)

Macrosiphum euphorbiae

Myzus (N.) persicae persicae [n.r.]

Pisum sativum (Fabaceae)

Acyrthosiphon kondoi

Acyrthosiphon pisum

Aphis craccivora craccivora

Aphis gossypii

Aulacorthum solani solani

Macrosiphum euphorbiae

Myzus (N.) persicae persicae

Smynthurodes betae

Pitraea cuneato-ovata (Verbenaceae)

Aphis gossypii [n.r.]

Eucarazzia elegans [n.r.]

Pittosporum tobira (Pittosporaceae)

Aphis fabae fabae

Plantago major (Plantaginaceae)

Myzus ornatus

Pluchea absinthioides (Asteraceae)

Aphis spiraecola [n.r.]

Macrosiphum euphorbiae

Uroleucon (L.) tessariae

Poa (Poaceae)

Rhopalosiphum padi

Sitobion avenae

Poa pratensis (Poaceae)

Metopolophium dirhodum

Rhopalosiphum padi
Schizaphis graminum

Sitobion avenae

Poaceae

Aulacorthum solani solani

Diuraphis noxia

Geoica lucifuga

Metopolophium dirhodum

Metopolophium festucae cerealium

Myzus (N.) persicae persicae

Rhopalosiphum maidis

Rhopalosiphum padi

Schizaphis graminum

Sitobion avenae

Sitobion fragariae

Podocarpus (Podocarpaceae)

Aphis spiraecola

Podocarpus salignus

Neophyllaphis (C.) podocarpini

Polygonaceae Brachycaudus (T.) amygdalinus

Polygonum persicaria: véase Persicaria maculosa

Polypogon (Poaceae)

Metopolophium dirhodum

Rhopalosiphum padi

Sitobion avenae

Polypogon australis

Rhopalosiphum padi [n.r.]

Sitobion avenae [n.r.]

Polypogon interruptus

Rhopalosiphum padi [n.r.]

Sitobion avenae [n.r.]

Polypogon lagascae

Metopolophium dirhodum

Polypogon linearis

Metopolophium dirhodum [n.r.]

Rhopalosiphum padi [n.r.]

Schizaphis graminum [n.r.]

Sitobion avenae [n.r.]

Polypogon monspeliensis

Sitobion avenae

Populus (Salicaceae)

Thecabius populimonilis

Tuberolachnus salignus

Populus deltoides

Chaitophorus leucomelas

Pemphigus bursarius

Populus nigra

Chaitophorus leucomelas

Pemphigus bursarius

Pemphigus populitransversus

Phloeomyzus passerinii

Pterocomma populeum

Prosopis tamarugo (Fabaceae)

Aphis craccivora craccivora

Prunus (Rosaceae)

Aphis spiraecola

Brachycaudus helichrysi

Brachycaudus (A.) schwartzi

Myzus (N.) persicae persicae

Rhopalosiphum padi

Prunus armeniaca

Brachycaudus (S.) persicae

Myzus (N.) persicae persicae

Prunus cerasus: véase Cerasus avium

Prunus divaricata

Brachycaudus helichrysi

Brachycaudus (A.) schwartzi 
Tabla 1.- (Continuación)

\section{Prunus domestica \\ Aphis spiraecola \\ Brachycaudus helichrysi \\ Brachycaudus (S.) persicae \\ Hyalopterus pruni \\ Myzus (N.) persicae persicae}

Prunus dulcis: véase Amygdalus comunnis

Prunus persica

Brachycaudus helichrysi

Brachycaudus (S.) persicae

Brachycaudus (A.) schwartzi

Myzus (N.) persicae persicae

Prunus salicina

Aphis spiraecola

Brachycaudus helichrysi

Brachycaudus (S.) persicae

Myzus (N.) persicae persicae

Pseudotsuga menziesi (Pinaceae)

Elatobium abietinum

Psidium guajava (Myrtaceae)

Aphis gossypii

Punica granatum (Lythraceae)

Aphis gossypii

Aphis spiraecola

Aulacorthum solani solani [n.r.]

Brachycaudus helichrysi

Macrosiphum euphorbiae [n.r.]

Myzus (N.) persicae persicae

Pyracantha coccinea (Rosaceae)

Erisoma lanigerum

Pyrus communis (Rosaceae)

Aphis craccivora craccivora

Aphis gossypii

Aphis spiraecola

Eriosoma lanigerum

Eriosoma (M.) pyricola

Quercus (Fabaceae)

Hoplocallis picta

Myzocallis (A.) castanicola castanicola

Tuberculatus (T'oides.) annulatus

Quercus cerris

Myzocallis boerneri

Quercus ilex

Myzocallis boerneri

Quercus nigra

Myzocallis (A.) castanicola castanicola [n.r.]

Quercus robur

Tuberculatus querceus

Quercus suber

Myzocallis (A.) castanicola castanicola

Myzocallis boerneri

Quillaja saponaria (Quillajaceae)

Aphis craccivora craccivora [n.r.]

Aphis marthae

Raphanus (Brassicaceae)

Myzus (N.) persicae persicae

Raphanus raphanistrum

Aphis spiraecola

Brevicoryne brassicae

Myzus (N.) persicae persicae

Raphanus sativus

Brevicoryne brassicae

Myzus (N.) persicae persicae

Smynthurodes betae [n.r.]

Rhododendron (Ericaceae)

Aphis spiraecola

Illinoia azaleae
Rhododendron japonicum

Illinoia (M.) lambersi [n.r.]

Ribes alpestre (Grossulariaceae)

Aphis fabae fabae [n.r.]

Robinia pseudoacacia (Fabaceae)

Aphis craccivora craccivora

Aphis craccivora pseudacaciae

Appendiseta robiniae

Macrosiphum euphorbiae [n.r.]

Rosa (Rosaceae)

Chaetosiphon $(P$.$) tetrarhodum$

Chaetosiphon (P.) thomasi

Macrosiphum rosae

Myzaphis rosarum

Myzus ornatus

Rhodobium porosum

Wahlgreniella nervata nervata

Rosa moschata

Macrosiphum rosae

Rubus (Rosaceae)

Aphis ruborum

Sitobion fragariae

Rubus idaeus

Aphis craccivora craccivora

Aphis rubicola

Aphis spiraecola

Rubus ulmifolius

Aphis ruborum

Rumex (Polygonaceae)

Aphis fabae fabae

Brachycaudus (T.) rumexicolens

Myzus (N.) persicae persicae

Ruta bracteosa (Rutaceae)

Aulacorthum solani solani

Macrosiphum euphorbiae

Salix (Salicaceae)

Tuberolachnus salignus

Salix babylonica

Cavariella aegopodii

Salix viminalis

Cavariella aegopodii

Tuberolachnus salignus

Sambucus (Caprifoliaceae)

Aphis sambuci

Sambucus nigra

Aphis sambuci

Schinus (Anacardiaceae)

Aphis schinifoliae

Schinus johnstoni

Aphis schinivora

Schinus molle

Aphis schinifoliae

Secale cereale (Poaceae)

Metopolophium dirhodum

Rhopalosiphum padi

Rhopalosiphum rufiabdominale

Schizaphis graminum

Sitobion avenae

Senecio (Asteraceae)

Aulacorthum solani solani

Brachycaudus helichrysi

Senecio bicolor subsp. cineraria

Myzus (N.) persicae persicae [n.r.]

Senecio filaginoides

Aphis papillosa

Aphis senecionicoides

Senecio smithii 
Tabla 1.- (Continuación)

\begin{tabular}{|c|c|}
\hline Myzus ornatus [n.r.] & Uroleucon sonchi \\
\hline Uroleucon (L.) nuble [n.r.] & Sorghum (Poaceae) \\
\hline Senecio yegua & Rhopalosiphum maidis \\
\hline Uroleucon (L.) muermosum [n.r.] & Sorghum bicolor \\
\hline Setaria (Poaceae) & Rhopalosiphum maidis \\
\hline Rhopalosiphum maidis & Schizaphis graminum \\
\hline Setaria parviflora & Sorghum halepense \\
\hline Sitobion avenae [n.r.] & Rhopalosiphum maidis \\
\hline Setaria verticillata & Rhopalosiphum padi \\
\hline Sitobion avenae & Schizaphis graminum \\
\hline Silybum marianum (Asteraceae) & Sipha flava \\
\hline Macrosiphum euphorbiae & Sitobion avenae \\
\hline Uroleucon (U'an.) aeneum & Sparmannia (Malvaceae) \\
\hline Simmondsia chinensis (Simmondsiaceae) & Myzus ornatus [n.r.] \\
\hline Aphis craccivora craccivora [n.r.] & Spiraea cantoniensis (Rosaceae) \\
\hline Aphis fabae fabae [n.r.] & Aphis spiraecola \\
\hline Macrosiphum euphorbiae [n.r.] & Macrosiphum euphorbiae [n.r.] \\
\hline Myzus (N.) persicae persicae [n.r.] & Stipa (Poaceae) \\
\hline Sinapis (Brassicaceae) & Metopolophium dirhodum \\
\hline Brevicoryne brassicae & Schizaphis graminum \\
\hline Sinapis arvensis & Sitobion avenae \\
\hline Brevicoryne brassicae & Stipa speciosa \\
\hline Sisyrinchium (Iridaceae) & Hysteroneura setaria \\
\hline Metopolophium dirhodum [n.r.] & Taraxacum officinale (Asteraceae) \\
\hline Sitobion avenae [n.r.] & Pemphigus bursarius \\
\hline Sisyrinchium graminifolium & Uroleucon (L.) macolai [n.r.] \\
\hline Metopolophium dirhodum [n.r.] & Thesaria absinthioides: véase Pluchea absinthioides \\
\hline Rhopalosiphum padi [n.r.] & Thuja (Cupressaceae) \\
\hline Schizaphis graminum [n.r.] & Cinara (Cu.) cupressi \\
\hline Solanum (Solanaceae) & Cinara (Cu.) tujafilina \\
\hline Aphis craccivora craccivora & Trevoa quinquenervia (Rhamnaceae) \\
\hline Myzus (N.) persicae persicae & Aphis conflicta \\
\hline Solanum lycopersicon & Trifolium (Fabaceae) \\
\hline Aphis gossypii & Acyrthosiphon kondoi \\
\hline Aulacorthum solani solani & Aphis craccivora craccivora \\
\hline Macrosiphum euphorbiae & Aphis gossypii \\
\hline Myzus (N.) persicae persicae & Aulacorthum solani solani \\
\hline Solanum melongena & Macrosiphum euphorbiae \\
\hline Macrosiphum euphorbiae & Myzus ornatus \\
\hline Myzus (N.) persicae persicae & Myzus (N.) persicae persicae \\
\hline Solanum muricatum & Therioaphis $(P-)$ trifolii \\
\hline Macrosiphum euphorbiae & Trifolium pratense \\
\hline Myzus (N.) persicae persicae [n.r.] & Acyrthosiphon pisum \\
\hline Solanum nigrum & Aphis craccivora craccivora \\
\hline Aphis craccivora craccivora & Aphis gossypii \\
\hline Solanum tuberosum & Aulacorthum solani solani \\
\hline Aphis craccivora craccivora & Brachycaudus helichrysi \\
\hline Aphis gossypii & Myzus ornatus \\
\hline Aulacorthum solani solani & Therioaphis $(P-)$ trifolii \\
\hline Macrosiphum euphorbiae & Trifolium repens \\
\hline Myzus (N.) persicae persicae & Acyrthosiphon pisum \\
\hline Neomyzus circunflexus & Aphis craccivora craccivora \\
\hline Rhopalosiphoninus latysiphon & Aphis gossypii \\
\hline Rhopalosiphum rufiabdominale & Aulacorthum solani solani \\
\hline Smynthurodes betae & Brachycaudus helichrysi \\
\hline Sonchus (Asteraceae) & Geoica lucifuga [n.r.] \\
\hline Hyperomyzus lactucae & Myzus ornatus \\
\hline Macrosiphum euphorbiae & Nearctaphis bakeri \\
\hline Uroleucon sonchi & Therioaphis $(P-)$ trifolii \\
\hline Sonchus asper & Trifolium subterraneum \\
\hline Hyperomyzus lactucae & Geoica lucifuga [n.r.] \\
\hline Macrosiphum euphorbiae & Tristerix (Loranthaceae) \\
\hline Nasonovia ribisnigri & Aphis conflicta \\
\hline Sonchus oleraceus & Triticum (Poaceae) \\
\hline Hyperomyzus carduellinus & Aploneura lentisci \\
\hline Hyperomyzus lactucae & Diuraphis noxia \\
\hline Nasonovia ribisnigri & Metopolophium dirhodum \\
\hline
\end{tabular}


Tabla 1.- (Continuación)

\begin{tabular}{|c|c|}
\hline Rhopalosiphum maidis & Verbena littoralis (Verbenaceae) \\
\hline Rhopalosiphum padi & Myzus ornatus [n.r.] \\
\hline Rhopalosiphum rufiabdominale & Veronica anagallis-aquatica (Plantaginaceae) \\
\hline Schizaphis graminum & Macrosiphum euphorbiae \\
\hline Sitobion avenae & Viburnum tinus (Adoxaceae) \\
\hline Triticum aestivum & Aphis spiraecola \\
\hline Diuraphis noxia & Vicia benghalensis (Fabaceae) \\
\hline Macrosiphum euphorbiae & Acyrthosiphon pisum \\
\hline Metopolophium dirhodum & Vicia faba \\
\hline Metopolophium festucae cerealium & Acyrthosiphon pisum \\
\hline Myzus (N.) persicae persicae & Aphis craccivora craccivora \\
\hline Rhopalosiphum maidis & Aphis fabae fabae \\
\hline Rhopalosiphum padi & Brachycaudus helichrysi \\
\hline Rhopslosiphum rufiabdominale & Macrosiphum euphorbiae \\
\hline Schizaphis graminum & Myzus (N.) persicae persicae \\
\hline Sitobion avenae & Smynthurodes betae \\
\hline Sitobion fragariae & Vicia sativa \\
\hline Triticum turgidum var. durum & Acyrthosiphon pisum \\
\hline Sitobion avenae [n.r.] & Aphis craccivora craccivora \\
\hline Tulipa (Liliaceae) & Myzus ornatus \\
\hline Dysaphis tulipae & Vicia villosa \\
\hline Macrosiphum euphorbiae & Acyrthosiphon pisum \\
\hline Myzus (N.) persicae persicae & Viola (Violaceae) \\
\hline Tweedia birostrata (Apocynaceae) & Myzus (Sciamyzus) ascalonicus \\
\hline Aphis nerii [n.r.] & Viola odorata \\
\hline Ulmus (Ulmaceae) & Myzus (N.) persicae persicae \\
\hline Eriosoma (M.) pyricola & Vitis vinifera (Vitaceae) \\
\hline Myzus (N.) persicae persicae [n.r.] & Aphis illinoisensis \\
\hline Tinocallis saltans & Aphis spiraecola \\
\hline Uncinia (Cyperaceae) & Vulpia megalura (Poaceae) \\
\hline Thripsaphis unciniae & Metopolophium dirhodum \\
\hline Urtica (Urticaceae) & Weigela (Caprifoliaceae) \\
\hline Microlophium carnosum & Aphis spiraecola \\
\hline Urtica urens & Zea mays (Poaceae) \\
\hline Myzus (N.) persicae persicae & Macrosiphum euphorbiae \\
\hline Vaccinium (Ericaceae) & Metopolophium dirhodum \\
\hline Aphis gossypii & Rhopalosiphum maidis \\
\hline Illinoia pepperi & Rhopalosiphum padi \\
\hline Vaccinium corymbosum & Schizaphis graminum \\
\hline Aphis gossypii & \\
\hline
\end{tabular}

se deben sin duda a la pequeña cantidad de prospecciones realizadas en ellas o a su inexistencia, aunque puede haber otras causas, que no es posible identificar con los datos disponibles.

\section{COMPONENTES ALÓCTONOS Y AUTÓCTONOS DE LA AFIDOFAUNA CHILENA}

De las 169 especies y subespecies de áfidos registrados hasta el momento en Chile 128 son alóctonas (el 75,74\%); la mayor parte de ellas de origen paleártico. En general sus plantas hospedadoras en Chile o muchas de ellas en el caso de los áfidos polífagos, son también de origen alóctono y muchas son cultivadas (cereales, frutales, plantas de huerta o de jardín).

Las especies autóctonas originarias de la región Neotropical son 41 (24,26\% del total); 25 de ellas $(14,79 \%$ del total) han sido citadas hasta ahora solamente en Chile y $14(8,28 \%)$ se conocen sólo en Chile y
Argentina (Tabla 2). Treinta y siete de ellas se distribuyen a partes iguales entre los géneros Aphis (Aphidinae), con 4 especies exclusivas de Chile, 8 compartidas con Argentina y 1 con Argentina y Bolivia, Uroleucon (Aphidinae), con 6 exclusivas de Chile, 3 compartidas con Argentina y 3 con Argentina y algún otro país, y Neuquenaphis (Spicaphidinae), con 10 especies exclusivas de Chile y 2 más compartidas con Argentina. Dos más son del género Neophyllaphis (Neophyllaphidinae) y otra de Thripsaphis (Saltusaphidinae), las tres exclusivas de Chile.

La cantidad de especies autóctonas sudamericanas citadas en cada Región está claramente en relación con el clima y la vegetación correspondiente, por ejemplo las distribuciones de las especies de Neuquenaphis están claramente relacionadas con las de las especies de Nothofagus, y se concentran en la mitad sur del País. Al norte de la Región IV solamente se han registrado 2 especies autóctonas: 
Tabla 2.- Relación de especies y subespecies de áfidos conocidos en Chile y en cada una de sus 15 Regiones. Primer tramo: cantidad de especies de cada en cada Región según los encabezamientos de fila. Segundo tramo: Presencia conocida en cada Región de cada especie o subespecie, agrupadas en taxones de nivel familia, con ordenación alfabética (nombre de subgéneros y autorías de especies y subespecies en el texto); columna NT: celdilla ocupada, especie nativa neotropical citada en Chile, Argentina, Bolivia, Brasil o Perú abreviadas a C, A, Bo, Br, P respectivamente; grupo de columnas Regiones: Regiones ordenadas de izquierda a derecha según su posición geográfica de Norte a Sur; en las celdillas, B cita bibliográfica, $\mathbf{N}$ cita nueva en este artículo, asterisco [*] cita en alguna de las provincias de la Región; columna t.R.: número total de regiones en las que se conoce cada especie o subespecie.

Table 2.- List of aphid species and subspecies known in Chile and in each of its fifteen Regions. PRIMER TRAMO (First block): species quantity in each Region as row headings. SEGUNDO TRAMO (Second block): Known presence of each species or subspecies in each Region, grouped for family-group taxa, in alphabetical order (for subgenera names and species and subspecies authorities see the text). Column NT: cell occupied, Neotropical native species recorded from Chile, Argentina, Bolivia, Brazil and Peru, respectively abbreviated C, A, Bo, Br, and P. Group of columns "Regiones": Chilean Regions ranged from left to right according to their geographical position from north to south; into cells: B, bibliographic record, $\mathbf{N}$, new record in this paper, asterisk [*] record in some province belonging to the Region. Column t.R.: Total number of Regions in which each species or subspecies is known.

\begin{tabular}{|c|c|c|c|c|c|c|c|c|c|c|c|c|c|c|c|c|c|}
\hline & & $\mathbf{X V}$ & $\mathbf{I}$ & II & III & IV & $\mathbf{v}$ & M & VI & VII & VIII & IX & XIV & $\mathbf{x}$ & $\mathbf{X I}$ & XII & \\
\hline \multicolumn{18}{|l|}{ PRIMER TRAMO } \\
\hline ... totales por Región ... & & 22 & 37 & 36 & 40 & 50 & 92 & 93 & 69 & 85 & 88 & 75 & 52 & 64 & 43 & 27 & \\
\hline ... con cita bibliográfica & & 16 & 29 & 23 & 34 & 48 & 82 & 75 & 57 & 72 & 78 & 67 & 49 & 62 & 30 & 14 & \\
\hline ...por cita nueva & & 6 & 8 & 13 & 6 & 2 & 10 & 18 & 12 & 13 & 10 & 8 & 3 & 2 & 13 & 13 & \\
\hline ...con mención de provincias & & 21 & 20 & 24 & 20 & 31 & 78 & 79 & 47 & 64 & 72 & 54 & 38 & 35 & 31 & 20 & \\
\hline ...sin mención de provincias & & 1 & 17 & 12 & 20 & 19 & 14 & 14 & 22 & 21 & 16 & 21 & 14 & 29 & 12 & 7 & \\
\hline ... autóctonas por Región & & 1 & 1 & 0 & 1 & 6 & 7 & 13 & 1 & 18 & 17 & 15 & 6 & 16 & 12 & 5 & \\
\hline \multicolumn{18}{|l|}{ SEGUNDO TRAMO } \\
\hline \multicolumn{18}{|l|}{$\begin{array}{l}\text { Aphidinae Aphidini } \\
\text { Aphidina }\end{array}$} \\
\hline Aphis alstroemeriae & C & & & & & & $\mathrm{B}^{*}$ & & & & & & & & & & 1 \\
\hline Aphis asclepiadis & & $\mathrm{B}^{*}$ & B & & & & & & & & & & & & & & 2 \\
\hline Aphis (T.) aurantii & & $\mathrm{B}$ & B & $B^{*}$ & B & B & $\mathrm{B}^{*}$ & B & $\mathrm{B}$ & B & $B^{*}$ & & & & & & 10 \\
\hline Aphis berberidorum & C, A & & & & & & & & & & & & & & $\mathrm{B}^{*}$ & & 1 \\
\hline Aphis carrilloi & C & & & & & & & & & $\mathrm{B}^{*}$ & & & & & & & 1 \\
\hline Aphis conflicta & C, A & & & & & & & $\mathrm{B}^{*}$ & & $\mathrm{~B}^{*}$ & $\mathrm{~B}^{*}$ & $\mathrm{~B}^{*}$ & & & & & 4 \\
\hline Aphis craccivora craccivora & & $\mathrm{B}^{*}$ & B & B & $\mathrm{B}^{*}$ & $\mathrm{~B}^{*}$ & $\mathrm{~B}^{*}$ & $B^{*}$ & $\mathrm{~B}^{*}$ & $\mathrm{~B}^{*}$ & $\mathrm{~B}^{*}$ & $\mathrm{~B}$ & & B & & & 12 \\
\hline $\begin{array}{l}\text { Aphis craccivora } \\
\text { pseudacaciae }\end{array}$ & & & & & & & & $B^{*}$ & & & & & & & & & 1 \\
\hline Aphis danielae & C, A & & & & & $\mathrm{B}^{*}$ & & & & & & & & & & & 1 \\
\hline Aphis fabae fabae & & $\mathrm{B}^{*}$ & $\mathrm{~B}^{*}$ & $\mathrm{~B}^{*}$ & $\mathrm{~B}^{*}$ & $\mathrm{~B}^{*}$ & $\mathrm{~B}^{*}$ & $\mathrm{~B}^{*}$ & $\mathrm{~B}^{*}$ & $\mathrm{~B}$ & $\mathrm{~B}^{*}$ & $\mathrm{~B}^{*}$ & & $\mathrm{~B}^{*}$ & & & 12 \\
\hline Aphis forbesi & & & $\mathbf{N}^{*}$ & & & & & & $\mathbf{N}^{*}$ & & $\mathbf{N}^{*}$ & & & & & & 3 \\
\hline Aphis gossypii & & $\mathrm{B}^{*}$ & $\mathrm{~B}^{*}$ & $\mathrm{~B}^{*}$ & $\mathrm{~B}$ & $\mathrm{~B}^{*}$ & $\mathrm{~B}^{*}$ & $\mathbf{N}^{*}$ & $\mathrm{~B}$ & $\mathrm{~B}^{*}$ & $\mathrm{~B}^{*}$ & B & $\mathrm{B}$ & B & & & 13 \\
\hline Aphis hederae & & & & & & & & $\mathrm{B}^{*}$ & & & & & $\mathrm{~B}^{*}$ & & & & 2 \\
\hline Aphis illinoisensis & & & & & B & B & $\mathrm{B}$ & B & B & & & & & $\mathrm{B}^{*}$ & & & 6 \\
\hline Aphis marthae & C & & & & & & $\mathrm{B}^{*}$ & $\mathrm{~B}^{*}$ & & $\mathrm{~B}^{*}$ & $\mathrm{~B}^{*}$ & & & & & & 4 \\
\hline Aphis maulensis & C & & & & & & & & & $\mathbf{B}^{*}$ & & & & & & & 1 \\
\hline Aphis nasturtii & & & & & & & & $\mathrm{B}^{*}$ & & & & & & & & & 1 \\
\hline Aphis nerii & & & & $\mathrm{B}^{*}$ & & & $\mathrm{~B}^{*}$ & $B^{*}$ & $\mathbf{N}^{*}$ & $\mathbf{N}^{*}$ & & & & $\mathrm{~B}^{*}$ & & & 6 \\
\hline Aphis papillosa & C, A & & & & & & & & & & & & & & $\mathrm{B}^{*}$ & & 1 \\
\hline Aphis patagonica & C, A & & & & & & & & & $\mathrm{B}^{*}$ & $\mathrm{~B}^{*}$ & $\mathbf{N}^{*}$ & & $\mathrm{~B}^{*}$ & $\mathrm{~B}^{*}$ & $\mathrm{~B}^{*}$ & 6 \\
\hline Aphis roberti & C, A & & & & & & & & & & & & & & $\mathrm{B}^{*}$ & & 1 \\
\hline Aphis rubicola & & & & & & & & & & & $\mathbf{N}^{*}$ & & & & & & 3 \\
\hline Aphis ruborum & & & & & & & $\mathrm{B}^{*}$ & & $\mathbf{N}^{*}$ & $\mathrm{~B}^{*}$ & $\mathrm{~B}^{*}$ & & & & & & 4 \\
\hline Aphis sambuci & & & & & & & & & & & & & $\mathrm{B}^{*}$ & & $\mathbf{N}^{*}$ & & 2 \\
\hline Aphis schinifoliae & $\begin{array}{l}\text { C, A, } \\
\text { Bo }\end{array}$ & & & & $\mathbf{N}^{*}$ & & & $\mathbf{N}^{*}$ & & $\mathrm{~B}^{*}$ & & & & & & & 3 \\
\hline Aphis schinivora & C, A & & & & & & & & & $\mathrm{B}^{*}$ & & & & & & & 1 \\
\hline Aphis senecionicoides & C, A & & & & & & & & & & & & & & $\mathbf{N}^{*}$ & & 1 \\
\hline
\end{tabular}


Tabla 2.-(Continuación)

\begin{tabular}{|c|c|c|c|c|c|c|c|c|c|c|c|c|c|c|c|c|}
\hline & $\mathbf{X V}$ & $\mathbf{I}$ & II & III & IV & $\mathbf{v}$ & $\mathbf{M}$ & VI & VII & VIII & IX & XIV & $\mathbf{x}$ & $\mathbf{X I}$ & XII & \\
\hline Aphis spiraecola & $\mathrm{B}^{*}$ & $\mathrm{~B}^{*}$ & B & $\mathrm{B}^{*}$ & $\mathrm{~B}^{*}$ & $\mathrm{~B}^{*}$ & $\mathrm{~B}^{*}$ & $B$ & $\mathrm{~B}^{*}$ & $\mathrm{~B}^{*}$ & $\mathrm{~B}^{*}$ & $B$ & $B$ & & & 13 \\
\hline \multicolumn{17}{|l|}{$\begin{array}{l}\text { Aphidinae Aphidini } \\
\text { Rhopalosiphina }\end{array}$} \\
\hline Hyalopterus pruni & & & & $\mathbf{N}^{*}$ & & & & & & $\mathbf{N}^{*}$ & & & & & & 2 \\
\hline Hysteroneura setariae & & & & & $\mathrm{B}^{*}$ & $\mathrm{~B}^{*}$ & & & & & & & & & & 2 \\
\hline Melanaphis donacis & $\mathrm{B}^{*}$ & $\mathrm{~B}^{*}$ & & $\mathrm{~B}^{*}$ & & $\mathrm{~B}^{*}$ & $\mathrm{~B}^{*}$ & $\mathrm{~B}^{*}$ & & & & & & & & 6 \\
\hline Rhopalosiphum maidis & $B^{*}$ & $\mathrm{~B}$ & $\mathrm{~B}^{*}$ & $\mathrm{~B}^{*}$ & $\mathrm{~B}^{*}$ & $\mathrm{~B}^{*}$ & $B^{*}$ & $B^{*}$ & $B$ & $B^{*}$ & $B$ & $B$ & $B$ & & & 13 \\
\hline Rhopalosiphum nymphaeae & & & $\mathbf{N}^{*}$ & & & $\mathrm{~B}^{*}$ & $\mathrm{~B}^{*}$ & & $\mathrm{~B}^{*}$ & $\mathrm{~B}^{*}$ & & $\mathbf{N}^{*}$ & & & & 6 \\
\hline Rhopalosiphum padi & $\mathrm{B}^{*}$ & $B$ & $\mathrm{~B}^{*}$ & B & $\mathrm{B}$ & $B^{*}$ & $\mathrm{~B}^{*}$ & $\mathrm{~B}^{*}$ & $\mathrm{~B}^{*}$ & $\mathrm{~B}^{*}$ & $\mathrm{~B}^{*}$ & $\mathrm{~B}^{*}$ & $\mathrm{~B}^{*}$ & $B$ & $B$ & 15 \\
\hline $\begin{array}{l}\text { Rhopalosiphum } \\
\text { rufiabdominale }\end{array}$ & & & & & $B$ & $\mathrm{~B}$ & $\mathrm{~B}$ & $\mathrm{~B}$ & $\mathrm{~B}^{*}$ & $\mathrm{~B}$ & & & & & & 6 \\
\hline Schizaphis graminum & & $B$ & $B$ & $\mathrm{~B}^{*}$ & $B$ & $\mathrm{~B}^{*}$ & $\mathrm{~B}^{*}$ & $\mathrm{~B}^{*}$ & $\mathrm{~B}^{*}$ & $B^{*}$ & $B$ & $B$ & $B$ & $B$ & $B$ & 14 \\
\hline \multicolumn{17}{|l|}{ Aphidinae Macrosiphini } \\
\hline Acyrthosiphon kondoi & & $\mathrm{B}^{*}$ & $\mathrm{~B}^{*}$ & $\mathrm{~B}^{*}$ & $\mathrm{~B}^{*}$ & $\mathrm{~B}^{*}$ & $B$ & $B$ & $\mathrm{~B}^{*}$ & $\mathrm{~B}^{*}$ & $\mathrm{~B}^{*}$ & $B$ & $B$ & $B$ & & 13 \\
\hline Acyrthosiphon malvae malvae & & $\mathbf{N}^{*}$ & & $\mathbf{N}^{*}$ & & $B^{*}$ & & & & $\mathrm{~B}^{*}$ & & & & & & 4 \\
\hline Acyrthosiphon pisum & & $\mathrm{B}$ & $B$ & B & $B$ & $\mathrm{~B}^{*}$ & $B^{*}$ & $B^{*}$ & $\mathrm{~B}^{*}$ & $B^{*}$ & $\mathrm{~B}^{*}$ & $\mathrm{~B}^{*}$ & $\mathrm{~B}^{*}$ & $B$ & $\mathbf{N}^{*}$ & 14 \\
\hline Aulacorthum solani solani & & & & & & $\mathrm{B}^{*}$ & $B^{*}$ & $B^{*}$ & B & $B^{*}$ & $\mathrm{~B}^{*}$ & & $B^{*}$ & & $\mathbf{N}^{*}$ & 8 \\
\hline $\begin{array}{l}\text { Brachycaudus }(T .) \\
\text { amygdalinus }\end{array}$ & & & & $\mathbf{N}^{*}$ & & & & & & & & & & & & 1 \\
\hline Brachycaudus helichrysi & $\mathrm{B}^{*}$ & & & $\mathrm{~B}$ & $\mathrm{~B}^{*}$ & $\mathrm{~B}^{*}$ & $\mathrm{~B}^{*}$ & $B^{*}$ & $\mathrm{~B}^{*}$ & $\mathrm{~B}^{*}$ & $\mathrm{~B}^{*}$ & $\mathrm{~B}^{*}$ & $\mathrm{~B}^{*}$ & $\mathbf{N}^{*}$ & $\mathbf{N}^{*}$ & 13 \\
\hline Brachycaudus (S.) persicae & & $B$ & $B$ & B & $\mathrm{B}$ & $\mathrm{B}^{*}$ & $\mathrm{~B}^{*}$ & $B^{*}$ & $B$ & $B$ & $B^{*}$ & & $B$ & & & 11 \\
\hline $\begin{array}{l}\text { Brachycaudus }(T .) \\
\text { rumexicolens }\end{array}$ & & & & & & $\mathbf{N}^{*}$ & & & & $B^{*}$ & & & & $\mathrm{~B}^{*}$ & & 3 \\
\hline Brachycaudus (A.) schwartzi & & & $\mathbf{N}^{*}$ & $\mathrm{~B}^{*}$ & $\mathrm{~B}^{*}$ & $\mathrm{~B}^{*}$ & $\mathrm{~B}^{*}$ & $\mathrm{~B}^{*}$ & $B ?$ & $\mathrm{~B} ?$ & $B^{*}$ & & & & & 9 \\
\hline $\begin{array}{l}\text { Brachycaudus }(A .) \\
\text { tragopogonis }\end{array}$ & & & & & & & & & & & & & & & & 0 \\
\hline Brevicoryne brassicae & & B & B & B & $B^{*}$ & $\mathrm{~B}^{*}$ & $\mathrm{~B}^{*}$ & $B$ & $B^{*}$ & $\mathrm{~B}^{*}$ & $\mathrm{~B}^{*}$ & $\mathrm{~B}^{*}$ & $\mathrm{~B}^{*}$ & $B$ & $B$ & 14 \\
\hline Capitophorus elaeagni & & & $\mathbf{N}^{*}$ & $\mathrm{~B}^{*}$ & $\mathrm{~B}$ & $\mathrm{~B}^{*}$ & $B^{*}$ & $\mathrm{~B}^{*}$ & $B$ & $\mathrm{~B}^{*}$ & $B$ & & $B$ & & & 10 \\
\hline $\begin{array}{l}\text { Capitophorus hippophaes } \\
\text { javanicus }\end{array}$ & & & & & & & & & $\mathrm{B}^{*}$ & & & & & & & 1 \\
\hline Cavariella aegopodii & & $\mathbf{N}^{*}$ & $\mathbf{N}$ & $\mathrm{B}$ & $\mathrm{B}^{*}$ & $\mathrm{~B}^{*}$ & $\mathrm{~B}^{*}$ & $B^{*}$ & $\mathrm{~B}^{*}$ & $\mathrm{~B}^{*}$ & $\mathrm{~B}^{*}$ & $\mathbf{N}^{*}$ & $\mathrm{~B}^{*}$ & $B^{*}$ & $\mathbf{N}^{*}$ & 14 \\
\hline Chaetosiphon $(P$.$) fragaefolii$ & & & & B & $B$ & $\mathrm{~B}^{*}$ & $\mathrm{~B}^{*}$ & $B$ & $\mathrm{~B}^{*}$ & $B^{*}$ & $B$ & & $\mathrm{~B}^{*}$ & & & 9 \\
\hline $\begin{array}{l}\text { Chaetosiphon }(P .) \\
\text { tetrarhodum }\end{array}$ & & & & & & $\mathrm{B}^{*}$ & & & $\mathbf{N}^{*}$ & & & & & & & 2 \\
\hline Chaetosiphon $(P$.$) thomasi$ & & & $\mathbf{N}^{*}$ & & & & & & & & $B$ & $\mathrm{~B}^{*}$ & $\mathrm{~B} ?$ & & & 4 \\
\hline Cryptomyzus ballotae & & & & & & $B^{*}$ & & & & & & & & & & 1 \\
\hline Diuraphis noxia & & & $\mathbf{N}^{*}$ & B & $B^{*}$ & $\mathrm{~B}^{*}$ & $B^{*}$ & $\mathrm{~B}^{*}$ & $\mathrm{~B}^{*}$ & $\mathrm{~B}^{*}$ & $\mathrm{~B}^{*}$ & $\mathrm{~B}^{*}$ & & & $\mathrm{~B}^{*}$ & 11 \\
\hline Dysaphis apiifolia apiifolia & & $\mathbf{N}^{*}$ & & & $\mathrm{~B}^{*}$ & $\mathrm{~B}^{*}$ & & & & $\mathrm{~B}^{*}$ & & & & & & 4 \\
\hline Dysaphis foeniculus & $\mathbf{N}^{*}$ & & & & & & & $\mathrm{~B}^{*}$ & & & & $\mathrm{~B}^{*}$ & & & & 3 \\
\hline Dysaphis lappae cynarae & & & & B & $B$ & B & $B^{*}$ & $B$ & B & $B$ & $B$ & $B$ & $B$ & & $\mathbf{N}^{*}$ & 11 \\
\hline Dysaphis tulipae & & & & & & $\mathrm{B}^{*}$ & $\mathrm{~B}$ & & & & & & & & & 2 \\
\hline Elatobium abietinum & & & & & & & & & & & $B$ & $\mathrm{~B}^{*}$ & $B$ & $\mathbf{N}^{*}$ & $\mathbf{N}^{*}$ & 5 \\
\hline Eucarazzia elegans & $\mathbf{N}^{*}$ & & & & $\mathrm{~B}^{*}$ & & & $\mathbf{N}^{*}$ & & & & & & & & 3 \\
\hline Hyadaphis foeniculi & & & & & & $\mathrm{B}^{*}$ & & & & & & & & & & 1 \\
\hline Hyadaphis passerinii & & & & & & $\mathbf{N}^{*}$ & $\mathbf{N}^{*}$ & $\mathbf{N}^{*}$ & $\mathbf{N}^{*}$ & & & & $\mathbf{N}^{*}$ & & $\mathbf{N}^{*}$ & 6 \\
\hline Hyperomyzus carduellinus & & & & & & & & & $\mathbf{N}^{*}$ & & & & & & & 1 \\
\hline Hyperomyzus lactucae & $\mathbf{N}^{*}$ & $\mathbf{N}^{*}$ & $\mathbf{N}^{*}$ & & & $B^{*}$ & $B$ & $B$ & $B$ & $\mathrm{~B}^{*}$ & $B$ & $B$ & $\mathrm{~B}^{*}$ & $B$ & $B$ & 13 \\
\hline Idiopterus nephrelepidis & & & & & & & $\mathbf{N}^{*}$ & & & $\mathrm{~B}^{*}$ & & & & & & 2 \\
\hline Illinoia azaleae & & & & & & & $\mathbf{N}^{*}$ & & & & & & & & & 1 \\
\hline Illinoia (M.) lambersi & & & & & & & & & & & & $\mathrm{B}^{*}$ & & & & 1 \\
\hline Illinoia morrisoni & & $\mathrm{B}^{*}$ & $\mathrm{~B}$ & B & $B$ & B & $B$ & $B$ & $B$ & $B$ & $B$ & $B$ & $B$ & & & 12 \\
\hline Illinoia pepperi & & & & & & $\mathbf{N}^{*}$ & & & & & & & & & & 1 \\
\hline
\end{tabular}


Tabla 2.- (Continuación)

\begin{tabular}{|c|c|c|c|c|c|c|c|c|c|c|c|c|c|c|c|c|c|}
\hline & & $\mathbf{X V}$ & $\mathbf{I}$ & II & III & IV & $\mathbf{v}$ & $\mathbf{M}$ & VI & VII & VIII & IX & XIV & $\mathbf{x}$ & $\mathbf{X I}$ & XII & \\
\hline Lipaphis pseudobrassicae & & & $\mathbf{N}^{*}$ & & $\mathbf{N}^{*}$ & $\mathrm{~B}^{*}$ & & & & $\mathbf{N}^{*}$ & $\mathbf{N}^{*}$ & $\mathrm{~B}^{*}$ & $\mathrm{~B}^{*}$ & & & & 7 \\
\hline Macrosiphoniella sanborni & & & & & & & $\mathrm{B}^{*}$ & $\mathbf{N}^{*}$ & & & & & $\mathrm{~B}^{*}$ & B & & & 4 \\
\hline $\begin{array}{l}\text { Macrosiphoniella tanacetaria } \\
\text { bonariensis }\end{array}$ & & & & & & & & & & & $\mathrm{B}^{*}$ & & & & & & 1 \\
\hline Macrosiphum euphorbiae & & $\mathrm{B}^{*}$ & $\mathrm{~B}^{*}$ & $\mathrm{~B}^{*}$ & $\mathrm{~B}$ & $\mathrm{~B}^{*}$ & $\mathrm{~B}^{*}$ & $\mathrm{~B}^{*}$ & $\mathrm{~B}^{*}$ & $\mathrm{~B}$ & $\mathrm{~B}^{*}$ & $\mathrm{~B}^{*}$ & B & $\mathrm{B}^{*}$ & $\mathrm{~B}^{*}$ & $\mathrm{~B}$ & 15 \\
\hline Macrosiphum rosae & & & $\mathrm{B}$ & $\mathrm{B}$ & $\mathrm{B}$ & $\mathrm{B}^{*}$ & $\mathrm{~B}^{*}$ & $B^{*}$ & $\mathrm{~B}$ & $\mathrm{~B}^{*}$ & $\mathrm{~B}^{*}$ & $\mathrm{~B}^{*}$ & $\mathrm{~B}^{*}$ & $\mathrm{~B}$ & $\mathrm{~B}^{*}$ & $\mathbf{N}^{*}$ & 14 \\
\hline Metopolophium dirhodum & & & B & $\mathrm{B}^{*}$ & $\mathrm{~B}^{*}$ & $\mathrm{~B}^{*}$ & $\mathrm{~B}^{*}$ & $\mathrm{~B}^{*}$ & $\mathrm{~B}^{*}$ & $\mathrm{~B}^{*}$ & $\mathrm{~B}^{*}$ & $\mathrm{~B}^{*}$ & $\mathrm{~B}^{*}$ & B & $\mathbf{N}^{*}$ & & 13 \\
\hline $\begin{array}{l}\text { Metopolophium festucae } \\
\text { cerealium }\end{array}$ & & & & & & & $\mathrm{B}^{*}$ & & & $\mathrm{~B}^{*}$ & $\mathrm{~B}^{*}$ & $\mathrm{~B}^{*}$ & & & & & 4 \\
\hline Microlophium carnosum & & & & & & & & & & & & & $\mathrm{B}^{*}$ & & & & 1 \\
\hline Myzaphis rosarum & & $\mathbf{N}^{*}$ & & & & & & $\mathrm{~B}^{*}$ & $\mathrm{~B}$ & $\mathrm{~B}$ & $\mathrm{~B}$ & $\mathrm{~B}^{*}$ & & B & & & 7 \\
\hline Myzus (S.) ascalonicus & & & & & & & & & & & & & $\mathrm{B}^{*}$ & & $\mathbf{N}^{*}$ & & 2 \\
\hline Myzus (S.) cymbalariae & & & & & & & $\mathrm{B}^{*}$ & & & & & & & & & & 1 \\
\hline Myzus ornatus & & $\mathrm{B}^{*}$ & $\mathrm{~B}^{*}$ & $\mathrm{~B}^{*}$ & $\mathrm{~B}$ & $\mathrm{~B}$ & $\mathrm{~B}^{*}$ & $\mathrm{~B}^{*}$ & $\mathrm{~B}^{*}$ & $\mathrm{~B}^{*}$ & $\mathrm{~B}^{*}$ & $\mathrm{~B}^{*}$ & $\mathrm{~B}^{*}$ & B & & & 13 \\
\hline $\begin{array}{l}\text { Myzus (N.) persicae } \\
\text { nicotianae }\end{array}$ & & & & & & & $\mathrm{B}^{*}$ & & $\mathrm{~B}^{*}$ & $\mathrm{~B}^{*}$ & $\mathrm{~B}^{*}$ & & & & & & 4 \\
\hline Myzus (N.) persicae persicae & & $B^{*}$ & B & $\mathrm{B}^{*}$ & $B^{*}$ & $B^{*}$ & $\mathrm{~B}^{*}$ & $\mathrm{~B}^{*}$ & $\mathrm{~B}^{*}$ & $B^{*}$ & $\mathrm{~B}^{*}$ & $\mathrm{~B}^{*}$ & $\mathrm{~B}^{*}$ & $B^{*}$ & $\mathrm{~B}$ & $\mathrm{~B}^{*}$ & 15 \\
\hline Nasonovia ribisnigri & & & & & & $\mathrm{B}^{*}$ & $\mathrm{~B}^{*}$ & $\mathbf{N}^{*}$ & $\mathbf{N}^{*}$ & $\mathrm{~B}$ & $\mathrm{~B}^{*}$ & $\mathbf{N}^{*}$ & $\mathbf{N}^{*}$ & & $\mathbf{N}^{*}$ & $\mathbf{N}^{*}$ & 10 \\
\hline Nearctaphis bakeri & & & & & & & $\mathrm{B}^{*}$ & $\mathrm{~B}^{*}$ & & & & $\mathbf{N}^{*}$ & & & & & 3 \\
\hline Neomyzus circunflexus & & & & & & & $\mathrm{B}^{*}$ & & & & & $\mathrm{~B}$ & & $\mathrm{~B}^{*}$ & & & 3 \\
\hline Neotoxoptera formosana & & & & & & & $\mathrm{B}^{*}$ & $B^{*}$ & $\mathbf{N}^{*}$ & & & & & & & $\mathbf{N}^{*}$ & 4 \\
\hline Neotoxoptera oliveri & & & & & & & & $\mathbf{N}^{*}$ & & & & & & & & & 1 \\
\hline Ovatus crataegarius & & & & & & & B & $\mathrm{B}^{*}$ & B & & $\mathrm{B}^{*}$ & B & & & & & 5 \\
\hline Pentalonia nigronervosa & & & $\mathbf{N}^{*}$ & & & & & & & & & & & & & & 1 \\
\hline $\begin{array}{l}\text { Pleotrichophorus } \\
\text { chrysanthemi }\end{array}$ & & & & & & & $\mathrm{B}^{*}$ & & & & & & & & & & 1 \\
\hline Pterocomma populeum & & & & & & & & $\mathbf{N}^{*}$ & $\mathbf{N}^{*}$ & & & $\mathrm{~B}^{*}$ & $\mathrm{~B}^{*}$ & & $\mathbf{N}^{*}$ & & 5 \\
\hline Rhodobium porosum & & & & & & & $\mathrm{B}^{*}$ & $\mathrm{~B}^{*}$ & & & & & & & & & 2 \\
\hline Rhopalosiphoninus latysiphon & & & & & & $\mathbf{N}^{*}$ & & $\mathrm{~B}^{*}$ & & $\mathbf{N}^{*}$ & $\mathbf{N}^{*}$ & $\mathbf{N}^{*}$ & & & & & 5 \\
\hline Sitobion avenae & & & & & B & $\mathrm{B}$ & $\mathrm{B}^{*}$ & $\mathrm{~B}^{*}$ & $\mathrm{~B}^{*}$ & $\mathrm{~B}^{*}$ & $\mathrm{~B}^{*}$ & $\mathrm{~B}^{*}$ & $\mathrm{~B}^{*}$ & $\mathrm{~B}^{*}$ & $\mathbf{N}^{*}$ & $\mathbf{N}^{*}$ & 12 \\
\hline Sitobion fragariae & & & & & & & $\mathrm{B}^{*}$ & $\mathrm{~B}^{*}$ & $\mathrm{~B}^{*}$ & $\mathrm{~B}^{*}$ & $\mathrm{~B}^{*}$ & $\mathrm{~B}^{*}$ & $\mathrm{~B}^{*}$ & & $\mathrm{~B}^{*}$ & & 8 \\
\hline Uroleucon (U'an.) aeneum & & & & & & & $\mathrm{B}^{*}$ & & & $\mathbf{N}^{*}$ & $\mathbf{N}^{*}$ & $\mathrm{~B}^{*}$ & & & & & 4 \\
\hline $\begin{array}{l}\text { Uroleucon ambrosiae } \\
\text { lizerianum }\end{array}$ & & $\mathrm{B}^{*}$ & & & & $\mathrm{~B}^{*}$ & $\mathrm{~B}^{*}$ & $\mathrm{~B}$ & B & $\mathrm{B}$ & $\mathrm{B}^{*}$ & $\mathrm{~B}$ & & $\mathrm{~B}^{*}$ & $\mathrm{~B}^{*}$ & $\mathrm{~B}$ & 11 \\
\hline Uroleucon (L.) bereticum & $\begin{array}{l}\mathrm{C}, \mathrm{A}, \\
\mathrm{Br}, \mathrm{P}\end{array}$ & & & & & & & & & $\mathrm{B}^{*}$ & $\mathrm{~B}^{*}$ & $\mathrm{~B}^{*}$ & & $\mathrm{~B}^{*}$ & & & 4 \\
\hline Uroleucon (L.) brevisiphon & C & & & & & & & $\mathrm{B}^{*}$ & & & $\mathrm{~B}^{*}$ & $\mathrm{~B}^{*}$ & & $\mathrm{~B}^{*}$ & & & 4 \\
\hline Uroleucon (L.) chilense & C & & & & & $\mathrm{B}^{*}$ & & $\mathrm{~B}^{*}$ & & & & & & & & & 2 \\
\hline Uroleucon (U'an.) compositae & & & & & & & $\mathbf{N}^{*}$ & & & & $\mathrm{~B}^{*}$ & $\mathrm{~B}^{*}$ & & & & & 3 \\
\hline Uroleucon (L.) erigeronense & & & & & & & $\mathrm{B}^{*}$ & $B^{*}$ & & $\mathrm{~B}^{*}$ & & & & & & & 3 \\
\hline Uroleucon (L.) essigi & C & & & & & $\mathrm{B}^{*}$ & & & & & & & & & & & 1 \\
\hline Uroleucon (L.) eumadiae & C & & & & & & & $\mathrm{B}^{*}$ & & & & & & & & & 1 \\
\hline Uroleucon (L.) macolai & $\begin{array}{l}\text { C, A, } \\
\text { Bo }\end{array}$ & & & & & $\mathrm{B}^{*}$ & $\mathrm{~B}^{*}$ & & & $\mathrm{~B}^{*}$ & $\mathrm{~B}^{*}$ & & & & & & 4 \\
\hline Uroleucon (L.) muermosum & C & & & & & & & & & & & $\mathbf{N}^{*}$ & & $\mathrm{~B}^{*}$ & & & 2 \\
\hline Uroleucon (L.) nuble & C & & & & & & & & & & $\mathrm{B}^{*}$ & & & & $B^{*}$ & & 2 \\
\hline Uroleucon (L.) petrohuense & C & & & & & & & & & & & & & $\mathrm{B}^{*}$ & & & 1 \\
\hline $\begin{array}{l}\text { Uroleucon (L.) } \\
\text { pseudomuermosum }\end{array}$ & C & & & & & & & & & & & $\mathbf{N}^{*}$ & & $\mathrm{~B}^{*}$ & & & 2 \\
\hline Uroleucon sonchi & & & & & & & $\mathrm{B}^{*}$ & $\mathbf{N}^{*}$ & & $\mathrm{~B}^{*}$ & $\mathrm{~B}^{*}$ & & & $\mathrm{~B}^{*}$ & & & 5 \\
\hline Uroleucon (L.) tessariae & C, A & $\mathrm{B}^{*}$ & $\mathrm{~B}^{*}$ & & & $\mathrm{~B}^{*}$ & & $\mathrm{~B}^{*}$ & $\mathrm{~B}^{*}$ & & & & & & & & 5 \\
\hline Uroleucon (L.) tucumani & $\begin{array}{c}\mathrm{C}, \mathrm{A}, \\
\mathrm{Br}\end{array}$ & & & & & $\mathrm{B}^{*}$ & & $\mathbf{N}^{*}$ & & & & & & & & & 2 \\
\hline
\end{tabular}


Tabla 2.-(Continuación)

\begin{tabular}{|c|c|c|c|c|c|c|c|c|c|c|c|c|c|c|c|c|}
\hline & $\mathbf{x V}$ & $\mathbf{I}$ & II & III & IV & $\mathbf{V}$ & $\mathbf{M}$ & VI & VII & VIII & IX & XIV & $\mathbf{x}$ & $\mathbf{X I}$ & XII & \\
\hline Wahlgreniella nervata nervata & & & $\mathbf{N}^{*}$ & & & & $\mathrm{~B}^{*}$ & & & & & & & $\mathrm{~B}^{*}$ & & 3 \\
\hline \multicolumn{17}{|l|}{$\begin{array}{l}\text { Calaphidinae } \\
\text { Panaphidini Myzocallidina }\end{array}$} \\
\hline Hoplocallis picta & & & & & & $\mathbf{N}^{*}$ & $\mathbf{N}^{*}$ & & & & & & & & & 2 \\
\hline Myzocallis boerneri & & & & & & $\mathrm{B}$ & $\mathrm{B}^{*}$ & $\mathrm{~B}^{*}$ & & B & & & & & & 4 \\
\hline $\begin{array}{l}\text { Myzocallis }(A .) \text { castanicola } \\
\text { castanicola }\end{array}$ & & & & & & $\mathrm{B}^{*}$ & $\mathbf{N}^{*}$ & $\mathrm{~B}$ & & $\mathbf{N}^{*}$ & & $\mathrm{~B}^{*}$ & & & & 5 \\
\hline Myzocallis coryli & & & & & $\mathrm{B}$ & & & $\mathrm{B}^{*}$ & $\mathrm{~B}^{*}$ & $\mathrm{~B}$ & $\mathrm{~B}^{*}$ & $\mathrm{~B}^{*}$ & B & & & 7 \\
\hline $\begin{array}{l}\text { Tuberculatus (T'oides.) } \\
\text { annulatus }\end{array}$ & & & & & & $\mathbf{N}^{*}$ & $\mathbf{N}^{*}$ & $\mathbf{N}^{*}$ & $\mathbf{N}^{*}$ & $\mathrm{~B}^{*}$ & $\mathrm{~B}^{*}$ & $\mathrm{~B}^{*}$ & & & & 7 \\
\hline Tuberculatus querceus & & & & & & & $\mathrm{B}^{*}$ & & & & & & & & & 1 \\
\hline \multicolumn{17}{|l|}{$\begin{array}{l}\text { Calaphidinae } \\
\text { Panaphidini Panaphidini }\end{array}$} \\
\hline Appendiseta robiniae & & & & & & & & $\mathbf{N}^{*}$ & $\mathrm{~B}^{*}$ & & & & & & & 2 \\
\hline Chromaphis juglandicola & & & & & $\mathrm{B}^{*}$ & $\mathrm{~B}^{*}$ & $\mathrm{~B}^{*}$ & $\mathrm{~B}^{*}$ & B & $\mathrm{B}$ & & & & & & 6 \\
\hline Pterocallis alni & & & & & & & & $\mathbf{N}^{*}$ & $\mathbf{N}^{*}$ & $\mathbf{N}^{*}$ & & $\mathrm{~B}^{*}$ & & & & 4 \\
\hline Takecallis arundinariae & & & & & & $\mathbf{N}^{*}$ & & $\mathrm{~B}^{*}$ & & & & & & & & 2 \\
\hline Takecallis taiwana & & $\mathbf{N}^{*}$ & $\mathbf{N}^{*}$ & & & & $\mathrm{~B}^{*}$ & & $\mathbf{N}^{*}$ & & & & & & & 4 \\
\hline Therioaphis $(P$.$) trifolii$ & & $\mathrm{B}^{*}$ & $\mathbf{N}^{*}$ & & & B & $\mathrm{B}^{*}$ & B & B & $\mathrm{B}^{*}$ & $\mathrm{~B}^{*}$ & & & & & 8 \\
\hline Tinocallis saltans & & & & & & & $\mathrm{B}^{*}$ & & & & & & & & & 1 \\
\hline \multicolumn{17}{|l|}{$\begin{array}{l}\text { Chaitophorinae } \\
\text { Chaitophorini }\end{array}$} \\
\hline Chaitophorus leucomelas & $\mathrm{B}^{*}$ & $\mathrm{~B}^{*}$ & $\mathrm{~B}$ & B & $\mathrm{B}$ & $\mathrm{B}^{*}$ & $\mathrm{~B}^{*}$ & $\mathrm{~B}^{*}$ & $\mathrm{~B}^{*}$ & $\mathrm{~B}^{*}$ & $\mathrm{~B}^{*}$ & & & & & 11 \\
\hline \multicolumn{17}{|l|}{ Chaitophorinae Siphini } \\
\hline Sipha flava & & & & & & $\mathbf{N}^{*}$ & $\mathrm{~B}^{*}$ & & & & & & & & & 2 \\
\hline Sipha $(R$.$) maydis$ & & & & & $\mathrm{B}^{*}$ & $\mathbf{N}^{*}$ & & & & & & & & & & 2 \\
\hline \multicolumn{17}{|l|}{ Drepanosiphinae } \\
\hline Drepanosiphum oregonense & & & & & & & & & & & & & $B^{*}$ & $\mathbf{N}^{*}$ & & 2 \\
\hline \multicolumn{17}{|l|}{$\begin{array}{l}\text { Eriosomatinae } \\
\text { Eriosomatini }\end{array}$} \\
\hline Eriosoma lanigerum & & $\mathrm{B}$ & B & $\mathrm{B}^{*}$ & $\mathrm{~B}$ & $\mathrm{~B}^{*}$ & $\mathrm{~B}^{*}$ & $\mathrm{~B}^{*}$ & $B^{*}$ & $\mathrm{~B}^{*}$ & $\mathrm{~B}^{*}$ & B & $\mathrm{B}$ & $\mathrm{B}$ & & 13 \\
\hline Eriosoma (M.) pyricola & & & & & & B & $\mathrm{B}^{*}$ & $\mathrm{~B}^{*}$ & $\mathrm{~B}^{*}$ & $B$ & $B$ & B & $\mathrm{B}$ & & & 8 \\
\hline \multicolumn{17}{|l|}{ Eriosomatinae Fordini } \\
\hline Aploneura lentisci & & & & & & & & $\mathrm{B}^{*}$ & $\mathbf{N}^{*}$ & $\mathbf{N}^{*}$ & & & & & & 3 \\
\hline Geoica lucifuga & & & & & & & & & & & & $\mathrm{B}^{*}$ & & & & 1 \\
\hline Smynthurodes betae & & $\mathrm{B}$ & & & & $\mathrm{B}^{*}$ & B & B & $\mathrm{B}$ & $\mathrm{B}$ & $\mathrm{B}^{*}$ & & $\mathrm{~B}^{*}$ & & & 8 \\
\hline \multicolumn{17}{|l|}{ Eriosomatinae Pemphigini } \\
\hline Pemphigus bursarius & & & & & & B & $\mathrm{B}$ & $\mathrm{B}^{*}$ & & & B & B & $\mathrm{B}$ & & & 6 \\
\hline Pemphigus populitransversus & & & & $\mathbf{N}^{*}$ & & & $\mathrm{~B}^{*}$ & & & & & & & & & 2 \\
\hline Prociphilus fraxinifolii & & & & & & & & $\mathbf{N}^{*}$ & & $\mathrm{~B}^{*}$ & & & & & & 2 \\
\hline Thecabius populimonilis & & & & & & $\mathrm{B}^{*}$ & & & & & & & & & & 1 \\
\hline \multicolumn{17}{|l|}{ Lachninae Eulachnini } \\
\hline Cinara cedri & & & & & & $\mathrm{B}^{*}$ & $\mathrm{~B}^{*}$ & & & & $\mathbf{N}^{*}$ & & & & & 3 \\
\hline Cinara (Cu.) cupressi & $\mathbf{N}^{*}$ & $\mathrm{~B}^{*}$ & $\mathrm{~B}$ & B & $\mathrm{B}$ & $\mathrm{B}^{*}$ & B & B & $\mathrm{B}^{*}$ & $\mathrm{~B}$ & B & $\mathrm{B}$ & B & B & $\mathrm{B}^{*}$ & 15 \\
\hline Cinara (Cu.) cupressivora & & & & & & B & $\mathrm{B}$ & B & $\mathrm{B}$ & B & $B$ & & $B$ & $B$ & & 8 \\
\hline Cinara $(\mathrm{Cu}$.$) fresai$ & & B & $\mathbf{N}^{*}$ & $\mathrm{~B}^{*}$ & $\mathbf{N}^{*}$ & $\mathrm{~B}^{*}$ & $\mathrm{~B}^{*}$ & $\mathrm{~B}$ & B & B & B & $\mathrm{B}^{*}$ & $\mathrm{~B}$ & $\mathrm{~B}$ & & 13 \\
\hline Cinara (Cu.) juniperi & & & & & & & & & & & B & & & & & 1 \\
\hline Cinara pilicornis & & & & & & & & & & & $\mathrm{B}^{*}$ & $\mathrm{~B}^{*}$ & & $\mathrm{~B}^{*}$ & $\mathbf{N}^{*}$ & 4 \\
\hline Cinara pinimaritimae & & & & & & & & & $\mathbf{N}^{*}$ & $\mathrm{~B}^{*}$ & $\mathrm{~B}^{*}$ & & & & & 3 \\
\hline Cinara (Cu.) tujafilina & & $\mathrm{B}$ & $\mathbf{N}^{*}$ & & & B & B & $\mathrm{B}^{*}$ & B & $B$ & $\mathrm{~B}$ & & $\mathrm{~B}$ & B & & 10 \\
\hline Essigella californica & & & & & & B & $\mathrm{B}^{*}$ & $\mathrm{~B}^{*}$ & $\mathrm{~B}^{*}$ & & & & & & & 4 \\
\hline Eulachnus rileyi & & & & & & $\mathbf{N}^{*}$ & $\mathbf{N}^{*}$ & & & $\mathrm{~B}^{*}$ & $\mathbf{N}^{*}$ & & B & & & 5 \\
\hline
\end{tabular}


Tabla 2.- (Continuación)

\begin{tabular}{|c|c|c|c|c|c|c|c|c|c|c|c|c|c|c|c|c|c|}
\hline & & $\mathbf{X V}$ & $\mathbf{I}$ & II & III & IV & $\mathbf{v}$ & $\mathbf{M}$ & VI & VII & VIII & IX & XIV & $\mathbf{x}$ & $\mathbf{X I}$ & XII & \\
\hline \multicolumn{18}{|l|}{ Lachninae Lachnini } \\
\hline Tuberolachnus salignus & & & $\mathrm{B}$ & $\mathrm{B}^{*}$ & $\mathrm{~B}$ & B & B & $\mathrm{B}^{*}$ & B & B & $\mathrm{B}$ & $\mathrm{B}^{*}$ & $\mathrm{~B}$ & B & $\mathrm{B}^{*}$ & $\mathrm{~B}$ & 14 \\
\hline \multicolumn{18}{|l|}{ Neophyllaphidinae } \\
\hline $\begin{array}{l}\text { Neophyllaphis (C.) } \\
\text { michelbacheri }\end{array}$ & C & & & & & & & & & & & & & $\mathrm{B}^{*}$ & & & 1 \\
\hline $\begin{array}{l}\text { Neophyllaphis (C.) } \\
\text { podocarpini }\end{array}$ & C & & & & & & & & & & & & $\mathrm{B}^{*}$ & & & & 1 \\
\hline \multicolumn{18}{|l|}{ Phloeomyzinae } \\
\hline Phloeomyzus passerinii & & $\mathbf{N}^{*}$ & & $\mathbf{N}^{*}$ & $\mathrm{~B}^{*}$ & $\mathrm{~B}$ & B & B & $\mathrm{B}^{*}$ & $\mathrm{~B}^{*}$ & & & & $\mathbf{N}^{*}$ & $\mathbf{N}^{*}$ & & 10 \\
\hline \multicolumn{18}{|l|}{ Saltusaphidinae } \\
\hline Thripsaphis unciniae & C & & & & & & & & & & $\mathrm{B}^{*}$ & & $\mathrm{~B}^{*}$ & & & & 2 \\
\hline \multicolumn{18}{|l|}{ 'Spicaphidinae } \\
\hline Neosensoriaphis parva & C & & & & & & & $\mathrm{B}^{*}$ & & & & $\mathrm{~B}^{*}$ & & & & & 2 \\
\hline Neuquenaphis bulbicauda & C & & & & & & & & & $\mathrm{B}^{*}$ & $\mathrm{~B}^{*}$ & $\mathrm{~B}^{*}$ & $\mathrm{~B}^{*}$ & $\mathrm{~B}^{*}$ & & $\mathrm{~B}^{*}$ & 6 \\
\hline Neuquenaphis (S.) chilensis & C & & & & & & & & & & $\mathrm{B}^{*}$ & $\mathrm{~B}^{*}$ & & $\mathrm{~B}^{*}$ & $\mathrm{~B}^{*}$ & & 4 \\
\hline Neuquenaphis edwardsi & C, A & & & & & & $\mathrm{B}^{*}$ & $\mathbf{N}^{*}$ & & $\mathbf{N}^{*}$ & $\mathrm{~B}^{*}$ & $\mathrm{~B}^{*}$ & $\mathrm{~B}^{*}$ & $\mathrm{~B}^{*}$ & $\mathbf{N}^{*}$ & $\mathrm{~B}^{*}$ & 9 \\
\hline Neuquenaphis (S.) essigi & C & & & & & & $\mathrm{B}^{*}$ & $\mathrm{~B}^{*}$ & & $\mathrm{~B}^{*}$ & $\mathbf{N}^{*}$ & & & & & & 4 \\
\hline Neuquenaphis michelbacheri & C & & & & & & $\mathrm{B}^{*}$ & & & $\mathrm{~B}^{*}$ & $\mathrm{~B}^{*}$ & $\mathrm{~B}^{*}$ & $\mathrm{~B}^{*}$ & $\mathrm{~B}^{*}$ & & & 6 \\
\hline Neuquenaphis neobulbicauda & C & & & & & & & & & & & & & $\mathrm{B}^{*}$ & & & 1 \\
\hline Neuquenaphis palliceps & C, A & & & & & & & $\mathbf{N}^{*}$ & & $\mathrm{~B}^{*}$ & $\mathrm{~B}^{*}$ & $\mathrm{~B}^{*}$ & & $\mathrm{~B}^{*}$ & $\mathbf{N}^{*}$ & $\mathrm{~B}^{*}$ & 7 \\
\hline Neuquenaphis schlingeri & C & & & & & & & & & $\mathrm{B}^{*}$ & $\mathrm{~B}^{*}$ & $\mathrm{~B}^{*}$ & & $\mathrm{~B}$ & $\mathrm{~B}^{*}$ & & 5 \\
\hline Neuquenaphis sensoriata & C & & & & & & $B^{*}$ & $\mathbf{N}^{*}$ & & $\mathrm{~B}^{*}$ & $\mathrm{~B}^{*}$ & $\mathrm{~B}^{*}$ & & $\mathrm{~B}$ & & & 6 \\
\hline Neuquenaphis similis & C & & & & & & & & & $\mathrm{B}^{*}$ & $\mathrm{~B}^{*}$ & $\mathrm{~B}^{*}$ & & $\mathrm{~B}^{*}$ & $\mathrm{~B}^{*}$ & $\mathbf{N}^{*}$ & 6 \\
\hline Neuquenaphis staryi & C & & & & & & & & & $\mathrm{B}^{*}$ & & & & & & & 1 \\
\hline Neuquenaphis valdiviana & C & & & & & & & & & & & & $\mathrm{B}^{*}$ & & $\mathrm{~B}^{*}$ & & 2 \\
\hline
\end{tabular}

Uroleucon tessariae en las Regiones XV y I y Aphis schinifoliae en la Región III, mientras que en el conjunto de las Regiones XI y XII y en el conjunto de las Regiones XIV y X se conocen respectivamente 13 y 19 especies autóctonas.

\section{Perspectivas de inCREMENTO Y MEjora del CATÁlogo CHILENO DE PULGONES}

En Chile se han citado aproximadamente dos terceras partes del número de pulgones citados hasta ahora en la Argentina. La mayoría de las especies alóctonas y una buena parte de las autóctonas citadas en Chile se conocen también en Argentina. Ambos países están situados en una misma franja latitudinal, pero su muy diferente superficie y la influencia del océano relacionada con la presencia de la cordillera andina producen notables diferencias en clima y vegetación. En estas diferencias se debe apoyar la diferente composición faunística real, que dista de estar completamente conocida en ambos países.

En cuanto a las especies nativas sudamericanas se mantendrán algunas de las diferencias ya constatadas; así no es probable que lleguen a encontrarse en Chile las especies de la subfamilia Lizeriinae que sí se hallan en Argentina y que haya especies de los géneros Aphis, Uroleucon, Neuquenaphis y Neophyllaphis que se encuentren sólo en una de las vertientes de los Andes, coincidentes prácticamente con los territorios de ambos países, manteniéndose la mayor riqueza en especies en Argentina.

En cuanto a las especies alóctonas la hipótesis más verosímil es que todas o casi todas lleguen a encontrarse en uno y otro país.

En consecuencia cabe pensar que la riqueza de la afidofauna chilena ha de ser mucho mayor. Un ejemplo de ello es la existencia de muestras en la colección del Natural History Museum de Londres de pulgones recogidos sobre Adesmia viscida, Baccharis linearis, Baccharis sp., Chloraea sp., Gaultheria mucronata, Luzuriaga radicans y Tropaeolum sp., que pueden pertenecer a especies no descritas aún.

Mejorar el conocimiento de la afidofauna nacional chilena y los de cada una de las regiones depende de las capturas que se puedan hacer y del trabajo de identificación posterior. Lo ideal sería que esas capturas se produjeran en campañas organizadas realizadas en zonas adecuadas. Pero también son importantes las capturas esporádicas hechas por entomólogos profesionales y aficionados así como por naturalistas 
Tabla 3.- Cantidad de especies y subespecies registradas en cada una de las provincias de Chile con citas de pulgones. A: por orden alfabético total; B: por Regiones de norte a sur y en éstas por orden alfabético.

Table 3.- Number of species and subspecies recorded in each province of Chile with aphid records. A: Total alphabetical order; B: Alphabetical order in each of the Regions, which are ordered from north to south.

\begin{tabular}{|c|c|c|c|c|c|}
\hline \multicolumn{3}{|c|}{$\mathbf{A}$} & \multicolumn{3}{|c|}{ B } \\
\hline PROVINCIA & REGIÓN & CANTIDAD & REGIÓN & PROVINCIA & CANTIDAD \\
\hline Aisén & XI & 1 & $x V$ & Arica & 20 \\
\hline Antártica Chilena & XII & 1 & & Parinacota & 2 \\
\hline Antofagasta & ॥ & 9 & 1 & El Tamarugal & 9 \\
\hline Arauco & VIII & 21 & & Iquique & 13 \\
\hline Arica & $X V$ & 20 & $\|$ & Antofagasta & 9 \\
\hline Bío Bío & VIII & 24 & & El Loa & 17 \\
\hline Cachapoal & $\mathrm{VI}$ & 44 & & Tocopilla & 1 \\
\hline Cardenal Caro & VI & 3 & III & Copiapó & 13 \\
\hline Cauquenes & VII & 17 & & Huasco & 12 \\
\hline Cautín & IX & 42 & IV & Choapa & 3 \\
\hline Chacabuco & M & 3 & & Elqui & 30 \\
\hline Chiloé & $x$ & 7 & & Limarí & 4 \\
\hline Choapa & IV & 3 & V & Isla de Pascua & 10 \\
\hline Coihaique & $X I$ & 20 & & Los Andes & 21 \\
\hline Colchagua & VI & 10 & & Marga Marga & 21 \\
\hline Concepción & VIII & 8 & & Petorca & 13 \\
\hline Copiapó & III & 13 & & Quillota & 48 \\
\hline Cordillera & $M$ & 19 & & San Antonio & 5 \\
\hline Curicó & VII & 21 & & $\begin{array}{l}\text { San Felipe de } \\
\text { Aconcagua }\end{array}$ & 23 \\
\hline El Loa & $\|$ & 17 & & Valparaíso & 36 \\
\hline El Tamarugal & 1 & 9 & M & Chacabuco & 3 \\
\hline Elqui & IV & 30 & & Cordillera & 19 \\
\hline General Carrera & $\mathrm{XI}$ & 11 & & Maipo & 6 \\
\hline Huasco & III & 12 & & Melipilla & 8 \\
\hline Iquique & 1 & 13 & & Santiago & 69 \\
\hline Isla de Pascua & $\mathrm{V}$ & 10 & & Talagante & 3 \\
\hline Limarí & IV & 4 & $\mathrm{VI}$ & Cachapoal & 44 \\
\hline Linares & VII & 20 & & Cardenal Caro & 3 \\
\hline Llanquihue & $x$ & 24 & & Colchagua & 10 \\
\hline Los Andes & V & 21 & VII & Cauquenes & 17 \\
\hline Magallanes & XII & 16 & & Curicó & 21 \\
\hline Maipo & $M$ & 6 & & Linares & 20 \\
\hline Malleco & IX & 34 & & Talca & 48 \\
\hline Marga Marga & V & 21 & VIII & Arauco & 21 \\
\hline Melipilla & $M$ & 8 & & Bío Bío & 24 \\
\hline Ñuble & VIII & 64 & & Concepción & 8 \\
\hline Osorno & $x$ & 23 & & Ñuble & 64 \\
\hline Palena & $x$ & 3 & IX & Cautín & 42 \\
\hline Parinacota & $X V$ & 2 & & Malleco & 34 \\
\hline Petorca & V & 13 & XIV & Ranco & 5 \\
\hline Quillota & V & 48 & & Valdivia & 37 \\
\hline Ranco & XIV & 5 & $x$ & Chiloé & 7 \\
\hline San Antonio & V & 5 & & Llanquihue & 24 \\
\hline $\begin{array}{l}\text { San Felipe de } \\
\text { Aconcagua }\end{array}$ & V & 23 & & Osorno & 23 \\
\hline Santiago & M & 69 & & Palena & 3 \\
\hline
\end{tabular}


Tabla 3.- (Continuación)

\begin{tabular}{lccccc}
\hline & A & & & B \\
\hline PROVINCIA & REGIÓN & CANTIDAD & REGIÓN & PROVINCIA & CANTIDAD \\
\hline Talagante & M & 3 & XI & Aisén & 1 \\
Talca & VII & 48 & & Coihaique & 20 \\
Tierra del Fuego & XII & 3 & General Carrera & 11 \\
Tocopilla & II & 1 & & Antártica Chilena & 1 \\
Última Esperanza & XII & 6 & & Magallanes & 16 \\
Valdivia & XIV & 37 & & Tierra del Fuego & 3 \\
Valparaíso & $\mathrm{V}$ & 36 & Última Esperanza & 6 \\
\hline
\end{tabular}

y amantes de la naturaleza en general. Para que el material capturado sea adecuado, debe estar fijado en etanol al $70 \%$ y tener unos buenos datos de captura, género (mejor especie) de la planta hospedadora y localidad y fecha de captura al menos, y de ser posible color en vida de los insectos, presencia de cera pulverulenta o filamentosa, parte de la planta colonizada, densidad de la colonia y presencia de hormigas mutualistas.

El incremento del conocimiento de la fauna en general, y también de la afidofauna puede lograrse actualmente gracias a la afición a la fotografía de la naturaleza que tienen muchas personas y a las facilidades que ofrecen las actuales tecnologías de la imagen y de la comunicación. Los pulgones de la mayoría de las especies se encuentran habitualmente en grupos y si no se ven amenazados casi ni se mueven; fotografiarlos resulta así un trabajo no demasiado complicado y el resultado obtenido suele ser alentador. Unas fotografías bien hechas (desde varios ángulos) y una información fiable de la planta hospedadora, pueden permitir a un especialista una identificación tentativa, siquiera a nivel de género o de grupo de géneros (Pérez Hidalgo et al., 2009). A partir de ese trabajo de identificación preliminar se podrá proceder a la recolección del material si no ha trascurrido mucho tiempo desde la toma de la fotografía y es factible retornar al lugar, y a su envío a un especialista para su preparación e identificación. Quienes puedan y deseen sumarse a este empeño pueden ponerse en contacto con los autores.

\section{Agradecimientos}

Georges Remaudière (fallecido cuando este catálogo se empezaba a elaborar) y los responsables de la custodia de la colección afídica del Muséum national d'Histoire naturelle de París facilitaron a uno de los autores (N. N.) el estudio de especímenes depositados en ella; el Director del servicio de colecciones del SAG autorizó a otro de los autores (F.C.) el estudio de las muestras conservadas en el Servicio; Roger L. Blackman y Paul A. Brown, del Natural History Museum de Londres, facilitaron muestras e informaron sobre los ejemplares de Andinaphis paradoxa depositados en esa institución; a todos ellos nuestro agradecimiento.
Nuestro reconocimiento también a los dos revisores que evaluaron el manuscrito, por sus sugerencias y minuciosas anotaciones.

\section{Referencias}

Aguilera, A. \& Ortega, F., 1994. Determinación de Brachycaudus helichrysi (Kaltenbach) (Homoptera: Aphididae) en leguminosas forrajeras para la IX Región de Chile. Agricultura Técnica (Chile), 54(1): 65-67.

Aguilera, A. \& Pacheco, C., 1995. Determinación de depredadores del pulgón del avellano europeo, Myzocallis coryli (Goeze) (Homoptera: Aphididae) en la IX Región de Chile. Revista Chilena de Entomología, 22: 17-19.

Aguilera, A., Pacheco, C. \& Guerrero, J., 1994. Fluctuación poblacional de Myzocallis coryli (Goeze) (Homoptera: Aphididae) en la IX Región de Chile. Revista Peruana de Entomología, 37: 105-106.

Aguilera, A., Rebolledo, R. R. \& Klein Koch, C., 2006a. Coccinélidos (Coleoptera) depredadores de Myzocallis coryli (Goeze), (Hemiptera: Aphididae) en la Araucanía, Chile. IDESIA (Chile), 24(1): 13-16. http://dx.doi. org/10.4067/S0718-34292006000100003

Aguilera, A., Rebolledo, R. R. \& Klein Koch, C., 2006 b. Ciclo vital de Adalia angulifera Mulsant (Coleoptera: Coccinellidae) sobre el pulgón del avellano europeo Myzocallis coryli (Goeze) (Hemiptera: Aphididae). Agricultura Técnica (Chile), 66(3): 312-317. http://dx. doi.org/10.4067/S0365-28072006000300010

Anónimo, 2010. Lista de especies de Aphidoidea reportadas para Chile. http://entomologia.utalca.cl/Reprints/ Apendiceafi.pdf [Consulta septiembre 2015].

Apablaza, J. U., 1973. Presencia de Macrosiphum (Sitobion) avenae (Fabricius) (Homoptera, Aphididae) en sementeras de trigo en Chile. Ciencia e Investigación agraria, 1(1): 69-70.

Apablaza, J. U. \& Tiska, V., 1973. Poblaciones de áfidos (Homoptera: Aphididae) en trigo de la zona central chilena. Revista Chilena de Entomología, 7: 173-181.

Astorga, I., 2014. Programa control biológico del pulgón del nogal, Chromaphis juglandicola (Hem.: Aphididae), mediante el uso del parasitoide Trioxys pallidus (Hym.: Braconidae). http://www.exponut.cl/presentaciones/2013/ Exitosa estrategia en control biologico del pulgon del nogal-Srta Ilania Astorga.pdf [Consulta septiembre 2015]. 
Baltra, R. E., 1979. Biología y control del pulgón lanígero de la raíz del peral (Eriosoma pyricola, Baker y Davidson). Tesis Ingeniero Agrónomo. Universidad de Chile. Santiago. 69 pp.

Basoalto, E., Barros, W., Muñoz, C. \& Fuentes-Contreras, E., 2005. Presencia de Sipha maydis Passerini (Hemiptera: Aphididae) en Chile. Resúmenes XXVII Congreso Nacional de Entomología: 54.

Beèche Cisternas, M., González Erazo, P., Sandoval Clavería, A., Ide Mayorga, S., Murillo, M. E., Jaques, L., Rothmann, S., González, H., Estay, S., Muñoz, C., Ferrada, R., Peralta, M., Bravo, C., Soevert, H., Canales, R., Torres, V., Zapata, M. \& Carrillo, M., 2004. Informe anual 2004, Subdepartamento de Vigilancia y control de plagas forestales y exóticas invasoras. Gobierno de Chile, Ministerio de Agricultura, Servicio Agrícola y Ganadero. Santiago. 116 pp.

Blackman, R. L., Brown, P. A., Ramírez, C. C. \& Niemeyer, H. M., 2003. Karyotype variation in the South American aphid genus Neuquenaphis (Hemiptera, Aphididae, Neuquenaphidinae). Hereditas, 138: 6-10. http://dx.doi. org/10.1034/j.1601-5223.2003.01692.x

Blackman, R. L. \& Eastop, V. F., 1994. Aphids on the World's Trees. An Identification and Information Guide. CAB International \& The Natural History Museum. Oxon (Reino Unido). 987 pp., 16 plates.

Blackman, R. L. \& Eastop, V. F., 2006. Aphids on the World's herbaceous Plants and Shrubs. An Identification and Information Guide. John Wiley and Sons \& The Natural History Museum. Chichester (Reino Unido). 2 vols., 1024 pp.

Blackman, R. L. \& Eastop, V. F., 2016. Aphids on the World's plants. An online identification and information guide. http://www.aphidsonworldsplants.info [Consulta febrero de 2016]

CAB International Institute of Entomology, 1986. Elatobium abietinum (Walker). Distribution Maps of Pests. Series A (Agricultural), 222: 1.

CAB International Institute of Entomology, 1989. Acyrthosiphon kondoi Shinji. Distribution Maps of Pests. Series A (Agricultural), 505: 1.

Caballero, P. P., Gonzales, W. L. \& Niemeyer, H. M., 2000. Tuberculatus querceus (Kaltenbach) (Hemiptera Aphididae): reporte de un nuevo áfido de la encina (Quercus robur) para Chile. Revista Chilena de Entomología, 27: 71-73.

Cabrera-Brandt, M. A., Fuentes-Contreras, E. \& Figueroa, C. C., 2010. Differences in the detoxification metabolism between two clonal lineages of the aphid Myzus persicae (Sulzer) (Hemiptera: Aphididae) reared on tobacco (Nicotiana tabacum L.). Chilean Journal of Agricultural Research, 70(4): 567-575. http://dx.doi. org/10.4067/S0718-58392010000400006

Caltagirone, L., 1957. Insectos entomófagos y sus huéspedes anotados para Chile. Agricultura Técnica (Chile), 17: $16-48$.

Campos, L., 1953. Plagas entomológicas de la Agricultura en Chile, Cartilla 49. Ministerio de Agricultura, Sanidad Vegetal. Santiago de Chile. 1 p.

Campos, L. \& Peña, L., 1973. Los insectos de Isla de Pascua. Revista Chilena de Entomología, 7: 217-229.
Capdeville, C., 1945. Plagas de la agricultura en Chile. Imprenta Pacífico. Valparaíso. 336 pp.

Carrillo, L. R., 1974. Aphidoidea de Chile. I. Agro Sur, 2(1): 33-40.

Carrillo, L. R., 1977. Aphidoidea de Chile II. Agro Sur, 5(2): 109-114.

Carrillo, L. R., 1980. Aphidoidea de Chile III. Agro Sur, 8(1): 21-29.

Carrillo, L. R. \& Mellado, M., 1975. Efecto de la época de siembra y del áfido Metopolophium dirhodum (Walker) en el rendimiento de cultivares de trigo de primavera (Triticum aestivium L.). Agricultura Técnica (Chile), 35(4): 190-204.

Carrillo, L. R., Mellado, M. \& Pino, A., 1974. Los áfidos Sitobion avenae (Fab.) y Metopolophium dirhodum (Walk.), su influencia en el rendimiento, ubicación en la planta y sus enemigos naturales. Agro Sur, 2(2): 71- 85.

Carrillo, L. R. \& Mundaca, B. N., 1995. Aphidoidea de Chile IV. Agro Sur, 23(2): 186-188.

Carrillo, L. R. \& Zúñiga, E., 1974. Nota científica. Clave para determinar las especies de áfidos (Homoptera: Aphididae) que se encuentran en cereales en Chile. Agro Sur, 2(2): 86-87.

Castañeda, L. E., Barrientos, K., Cortés, P. A., Figueroa, C. C., Fuentes-Contreras, E., Luna-Rudolf, M., Silva, A. X. \& Bacigalupe, L. D., 2011. Evaluating reproductive fitness and metabolic costs for insecticide resistance in Myzus persicae from Chile. Physiological Entomology, 36: 253-260. http://dx.doi. org/10.1111/j.1365-3032.2011.00793.x

Charlín, C. R., 1991. El pulgón de la raíz de las compuestas en huertos de frutales. Aconex, 31: 11-15.

Commonwealth Institute of Entomology, 1964a. Pest: Toxoptera aurantii (Boy.). Distribution Maps of Pests. Series A (Agricultural), 13: 1.

Commonwealth Institute of Entomology, 1964b. Pest: Toxoptera graminum (Rond.). Distribution Maps of Pests. Series A (Agricultural), 173: 1.

Commonwealth Institute of Entomology, 1968. Pest: Aphis gossypii Glover. Distribution Maps of Pests. Series A (Agricultural), 18 (Revised): 1.

Commonwealth Institute of Entomology, 1969. Pest: Aphis spiraecola Patch. Distribution Maps of Pests. Series A (Agricultural), 256: 1.

Commonwealth Institute of Entomology, 1971a. Pest: Rhopalosiphum maidis (Fitch). Distribution Maps of Pests. Series A (Agricultural), 67 (Revised): 1-3.

Commonwealth Institute of Entomology, 1971b. Pest: Rhopalosiphum rufiabdominalis (Sasaki). Distribution Maps of Pests. Series A (Agricultural), 289: 1.

Commonwealth Institute of Entomology, 1971c. Pest: Rhopalosiphum padi (L.). Distribution Maps of Pests. Series A (Agricultural), 288: 1.

Commonwealth Institute of Entomology, 1975. Pest: Eriosoma lanigerum (Haussman.). Distribution Maps of Pests. Series A (Agricultural), 17 (revised): 1-3.

Commonwealth Institute of Entomology, 1979. Pest: Eriosoma lanigerum (Hsm.). Distribution Maps of Pests. Series A (Agricultural), 17 (Revised): 1. 
Commonwealth Institute of Entomology, 1986. Aulacorthum solani (Kaltenbach). Distribution Maps of Pests. Series A (Agricultural), 86: 1-3

Cortázar Sagarminaga, R., 1980. Virus del enanismo amarillo de la cebada (BYDV) y áfidos en trigo en la región centro-norte de Chile. Agricultura Técnica (Chile), 40(2): 53-57.

de Carvalho, R. C. Z., Blackman, R. \& Spence, J. M., 1998. The genus Uroleucon Mordvilko (Insecta, Aphidoidea) in South America, with a key and descriptions of four new species. Zoological Journal of the Linnean Society, 123: 117-141. http://dx.doi.org/10.1111/j.1096-3642.1998.tb01296.x

Delfino, M. A. \& Gonzales, W. L., 2005. A New Species of Uroleucon (Hemiptera: Aphididae) on Madia (Asteraceae) in Chile. Neotropical Entomology, 34(2): 221-225. http://dx.doi.org/10.1590/S1519-566X2005000200010

Eastop, V. F., Heie, O. E., Fuentes-Contreras, E., Petterson, J. \& Niemeyer, H. M., 1997. Notes on two new aphid species (Hemiptera: Aphididae) detected in Chile. Revista Chilena de Entomología, 24: 24-81.

Essig, E. O., 1953. Some new and noteworthy Aphididae from Western and Southern South America. Proceedings of the Californian Academia of Sciences (Fourth Series), 28(3): 59-164.

Favret, C., 2014. Aphid Species File. Versión 5.0 http:// aphid.speciesfile.org [Consulta septiembre 2014].

Forestry Department FAO, 2008. Overview of Forest Pest, Chile. FAO. Roma (Italia). 4+30 pp.

Fuentes-Contreras, E., Basoalto, E., Sandoval, C., Burgos, R., Leal, C., Pávez, P. \& Muñoz, C., 2007. Evaluación de la eficacia, efecto residual y de volteo de aplicaciones en pretrasplante de insecticidas nicotinoides y mezclas de nicotinoide-piretroide para el control de Myzus persicae (Hemiptera: Aphididae) en tabaco. Agricultura Técnica (Chile), 67(1): 16-22. http://dx.doi.org/10.4067/ S0365-28072007000100002

Fuentes-Contreras, E., Figueroa, C. C., Reyes, M., Briones, L. M. \& Niemeyer, H. M., 2004. Genetic diversity and insecticide resistance of the Myzus persicae (Hemiptera: Aphididae) populations from tobacco in Chile: evidence for the existence of a single predominant clone. Bulletin of Entomological Research, 94(1): 11-18. http://dx.doi. org/10.1079/BER2003275

Fuentes-Contreras, E., Muñoz, R. \& Niemeyer, H. M., 1997. Diversidad de áfidos (Hemiptera: Aphidoidea) en Chile. Revista Chilena de Historia Natural, 70: 531-542.

Gaete-Eastman, C., Figueroa, C. C., Olivares-Donoso, R., Niemeyer, H. M. \& Ramírez, C. C., 2004. Diet breadth and its relationship with genetic diversity and differentiation: the case of Southern beech aphids (Hemiptera, Aphididae). Bulletin of Entomological Research, 94: 219-227. http://dx.doi.org/10.1079/BER2004298

Gerding, M. \& Figueroa, A., 1989. Reducción de la progenie de Sitobion avenae (Homoptera: Aphididae) atribuida a Aphidius ervi (Hymenoptera: Aphidiidae). Agricultura Técnica (Chile), 49(1): 50-53.

Gerding, M. \& Norambuena, M., 1991. Posible rol de los enemigos naturales de áfidos presentes en Chile sobre el áfido ruso del trigo (Diuraphis noxia Mordvilko) (Homoptera: Aphididae). Agricultura Técnica (Chile), 51(1): 69-71.
Gerding, M., Zúñiga, E., Quiroz, C., Norambuena, H. \& Vargas, R., 1989. Abundancia relativa de los parasitoides de Sitobion avenae (F) y Metopolophium dirhodum (Wlk) (Homoptera: Aphididae) en diferentes áreas geográficas de Chile. Agricultura Técnica (Chile), 49(2): 104-114.

Gonzales, L. W., Fuentes-Contreras, E. \& Niemeyer, H. M., 1998a. Registro de un nuevo áfido introducido en Chile: Takecallis taiwanus (Takahashi) (Hemiptera: Aphididae: Drepanosiphinae). Revista Chilena de Entomología, 26: 53-55.

Gonzales, L. W., Fuentes-Contreras, E. \& Niemeyer, H. M., 1998b. Una nueva especie de áfido (Hemiptera: Aphididae) detectada en Chile: Sipha flava (Forbes). Revista Chilena de Entomología, 25: 87-90.

González, R., 2011. Manejo de polillas y del pulgón del nogal. http://www.chilenut.cl/exponut/assets/docs/ presentaciones/el_pulgon_y_la_polilla_del_nogal, dos_nuevas_plagas_para_chile-roberto_gonzalez.pdf [Consulta julio 2014].

Heie, O. E., Petterson, J., Fuentes-Contreras, E. \& Niemeyer, H. M., 1996. New records of aphids (Hemiptera: Aphidoidea) and their host-plants from Northern Chile. Revista Chilena de Entomologia, 23: 83-87.

Henríquez, M. A., 1991. Control del pulgón de la ráiz del peral (Eriosoma pyricola Baker y Davidson) mediante tratamientos foliares y al suelo. Tesis Ingeniero Agrónomo. Universidad de Chile. Santiago. 44 pp.

Herrera, G. \& Quiroz, C., 1980. Efecto del virus del enanismo amarillo de la cebada ("Barley Yellow Dwarf Virus") y del áfido Metopolophium dirhodum Walker en trigo (Triticum aestivum L.). Agricultura Técnica (Chile), 40(1): 12-17.

Hille Ris Lambers, D., 1968. A study of Neuquenaphis Blanchard (E.E.), 1939, with descriptions of new species (Aphididae, Homoptera). Tijdschrift voor Entomologie, 111(7): 257-286.

Hille Ris Lambers, D., 1975. Observaciones sobre el problema de los áfidos en Chile. Boletín Técnico, 9: 4-15.

Holman, J., 2009. Host Plant Catalog of aphids, Palaerctic Region. Springer Science + Busines Media B.V. [Sin localidad] Alemania. 4+1616 pp.

International Institute of Entomology, 1991. Diuraphis noxia (Mordvilko). Distributions Maps of Pests. Series A (Agricultural), 521: 1-3.

Klein Koch, C. \& Waterhouse, D. F., 2000. The distribution and importance of arthropods associated with agricultura and forestry in Chile / Distribución e importancia de los artrópodos asociados a la agricultura y silvicultura en Chile. Australian Centre for International Agricultural Research, Monograph No. 68. Camberra. 234 pp.

Lagos, D. M., Voegtlin, D. J., Coeur d'acier, A. \& Giordano, R., 2014. Aphis (Hemiptera: Aphididae) species groups found in the Midwestern United States and their contribution to the phylogenetic knowledge of the genus. Insect Science, 21: 374-391. http://dx.doi. org/10.1111/1744-7917.12089

Lagos-Kutz, D., Favret, C., Giordano, R. \& Voegtlin, D. J., 2016. The status of the members of the Aphis asclepiadis species group (Hemiptera: Aphididae) in the United States of America. Annals of the Entomological Society 
of America, 109(4): 585-594. http://dx.doi.org/10.1093/ aesa/saw020

Lara, S. \& Zúñiga, E., 1969. Metopolophium dirhodum (Walker) (Homoptera, Aphididae). Áfido nuevo para Chile, importante plaga del trigo. Simiente, 39(1-3): 34-36.

Lavandero, B., Figueroa, C. C., Franck, P. \& Méndez, A., 2011. Estimating gene flow between refuges and crops: a case study of the biological control of Eriosoma lanigerum by Aphelinus mali in apple orchards. PLoS ONE, 6(11): e26694. http://dx.doi.org/10.1371/journal. pone.0026694

Lavandero, B., Ramírez, C.C., Miranda, M. \& FuentesContreras, E., 2009. Landscape composition modulates population genetic structure of Eriosoma lanigerum (Hausmann) on Malus domestica Borkh. in Central Chile. Bulletin of Entomological Research, 99: 97-105. http://dx.doi.org/10.1017/S0007485308006196

Lavandero, B., Rojas, P., Ramírez, C. C., Salazar, M. \& Caligari, P. D. S., 2012. Genetic structure of the aphid Chaetosiphon fragaefolii, and its role as a vector of the Strawberry Yellow Edge Virus to a native strawberry, Fragaria chiloensis in Chile. Journal of Insect Science, 12(110): 13 pp.http://dx.doi.org/10.1673/031.012.11001

Le Feuvre, R., 1875. Peste de los manzanos. Boletín de la Sociedad Nacional de Agricultura, 7(3): 63-64.

López Ciruelos, S. I., Mier Durante, M. P., Ortego, J., GarcíaTejero, S. \& Nieto Nafría, J. M., 2016. Three new South American species of genus Aphis (Hemiptera: Aphididae) living on species of Euphorbia (Euphorbiaceae). Zootaxa, 4085 (1): 103-118. http://dx.doi.org/10.11646/ zootaxa.4085.1.4

Luppichini, P. \& Ripa, R., 2011. Dos nuevas plagas asociadas al nogal. Tierra Adentro, 94: 25-27.

Machuca, M., 2014. Análisis técnico de la temporada de nogal. Redagricola, julio 2014. http://www.redagricola. com/reportajes/frutales/análisis técnico-de-la-temporada-de-nogal-2011-2012 [Consulta julio 2014]

Mier Durante, M. P., Nieto Nafría, J. M. \& Ortego, J., 2003. Aphidini (Hemiptera: Aphididae) living on Senecio (Asteraceae), with descriptions of a new genus and three new species. The Canadian Entomologist, 135: 187-212. http://dx.doi.org/10.4039/n02-065

Montalva, C., Rojas, E., Ruiz, C. \& Lanfranco, D., 2010. El pulgón del ciprés en Chile: una revisión de la situación actual y antecedentes del control biológico. Bosque, 31(2): 81-88.

Mújica, F., 1941. Nómina de las enfermedades y pestes de la papa cuya existencia se ha comprobado en el país. Boletín del Departamento de Sanidad Vegetal (Chile), 1(1): 70-72.

Muñoz, R., 1995. Nota sobre tres áfidos (Homoptera: Aphididae) nuevos para Chile. Revista Chilena de Entomología, 22: 85-88.

Muñoz, R. \& Beèche, M., 1995. Antecedentes sobre dos especies de reciente identificación para Chile (Homoptera: Aleyrodidae, Aphididae). Revista Chilena de Entomología, 22: 89-91.

Niemeyer, H.M., Bustamante, R. O., Simonetti, J. A. Teillier, S., Fuentes-Contreras, E. \& Mella, J.E., 2002.
Historia Natural de la Reserva Nacional Río Clarillo: un espacio para aprender ecología. Impresos Socías. Santiago de Chile. 310 pp.

Nieto Nafría, J. M. \& Favret, C., 2011. Preface / Prefacio. In: J. M. Nieto Nafría \& C. Favret (eds.). Registers of family-group and genus-group taxa of Aphidioidea / Registros de los taxones de nivel familia y de nivel género de Aphidoidea (Hemiptera Sternorrhyncha). Universidad de León. León: 11-20.

Nieto Nafría, J. M., Favret, C., Akimoto, S.-i., Barbagallo, S., Chakrabarti, S., Mier Durante, M. P., Miller, G. L., Qiao G.-X., Sano, M., Pérez Hidalgo, N., Stekolshchikov, A. V. \& Wegierek, P., 2011. Register of genus-group taxa of Aphidoidea / Registro de los taxones del nivel género de Aphidoidea. In: J. M. Nieto Nafría \& C. Favret (eds.). Registers of Family-group and Genus-group taxa of Aphidioidea / Registros de los taxones de nivel familia y de nivel género de Aphidoidea (Hemiptera Sternorrhyncha). Universidad de León. León: 81-404.

Nieto Nafría, J. M., Fuentes-Contreras, E. \& Pérez Hidalgo, N., 2015. Aphis (Toxoptera) citricidus (Kirkaldy) [Hemiptera: Aphididae] and Chile. Bulletin OEPP/EPPO Bulletin, 45(1): 1-4. http://dx.doi.org/10.1111/epp.12181

Nieto Nafría, J. M., Mier Durante, M. P., Ortego, J. \& Seco Fernández, M. V., 2007. The genus Uroleucon (Hemiptera: Aphididae: Macrosiphini) in Argentina, with descriptions of five new species. The Canadian Entomologist, 139: 154-178. http://dx.doi.org/10.4039/n05-100

Nieto Nafría, J. M., Ortego, J. \& Mier Durante, M. P., 1999. Three new species of Aphis (Hemiptera: Aphididae) living on Mulinum (Umbelliferae) in South America. The Canadian Entomologist, 131(3): 283-292. http:// dx.doi.org/10.4039/Ent131283-3

Nieto Nafría, J. M., Ortego, J. \& Mier Durante, M. P., 2008. The South American species of Aphis (Hemiptera: Aphididae: Aphidina) living on Rhamnaceae and Loranthaceae, with description of a new species. Proceedings of the Entomological Society of Washington, 110(2): 481-492. http://dx.doi.org/10.4289/07-039.1

Norambuena, M. H. \& Gerding, P. M., 1991. Actual distribución en Chile del áfido ruso del trigo Diuraphis noxia Mordvilko (Homoptera: Aphididae). Agricultura Técnica (Chile), 51(1): 65-68.

Olalquiaga Fauré, G., 1944. Identificaciones y datos adicionales de algunos insectos y arácnidos de Chile. Revista Chilena de Historia Natural, 48(1): 66-76.

Olalquiaga Fauré, G., 1946. Notas sistemáticas y biológicas para la entomología de Chile. Agricultura Técnica (Chile), 6(1): 74-78.

Ortego, J., 1998. Pulgones de la Patagonia Argentina con la descripción de Aphis intrusa sp. n. (Homoptera: Aphididae). Revista de la Facultad de Agronomía (La Plata), 102(1): 59-80.

Ortego, J., Difabio, M. E. \& Mier Durante, M. P., 2004. Nuevos registros y actualización de la lista faunística de los pulgones (Hemiptera: Aphididae) de la Argentina. Revista de la Sociedad Entomológica Argentina, 63 (1-2): 19-30.

Ortego, J. \& Mier Durante, M. P., 1997. Les espèces sud-américaines d'Aphis inféodées au genre Berberis 
(Hemiptera: Aphididae). Annales de la Societé Entomologique de France (Nouvelle Serie), 33(4): 411-418.

Ortego, J., Mier Durante, M. P. \& Nieto Nafría, J. M., 2013. Una nueva especie de Aphis (Hemiptera: Aphididae) sobre Gunnera magellanica (Gunnerales: Gunneraceae) de la región del Maule (Chile). Revista Chilena de Entomología, 38: 23-32.

Ortego, J., Nieto Nafría, J. M. \& Mier Durante, M. P., 2007. Species of Aphis (Hemiptera: Aphididae: Aphidina) living on Schinus (Sapindales: Anacardiaceae) with description of a new species. Proceedings of the Entomological Society of Washington, 109(2): 338-394.

Peccoud, J., Figueroa, C. C., Silva-Báez, A., Ramírez, C. C., Mieuzet, L., Bonhomme, J., Stoeckel, S., Plantegenest, M. \& Simon, J. C., 2008. Host range expansion of an introduced insect pest in Chile through multiple colonisations of specialized clones. Molecular Ecology, 17: 4608-4618. http://dx.doi.org/10.1111/j.1365-294X.2008.03949.x

Philippi, R. A., 1885. Sobre los animales introducidos en Chile, desde su conquista por los españoles. Anales de la Universidad de Chile, 67: 319-335.

Porter, C. E., 1916. Un pajarillo destructor de pulgones. Anales de Zoología Aplicada, 3(1): 30.

Porter, C. E., 1917. Sobre el régimen de algunos artrópodos de importancia económica. Anales de Zoología Aplicada, 4(2): 37-38.

Porter, C. E., 1922. Notas breves de entomología aplicada. Anales de Zoología Aplicada, 9: 36-37.

Porter, C. E., 1923. Un microhymenóptero parásito del pulgón lanígero. Revista Chilena de Historia Natural, 27: 220.

Prado, E. C., 1991. Artrópodos y sus enemigos naturales asociados a plantas cultivadas en Chile. Boletín Técnico del Instituto de Investigaciones Agropecuarias, 169: 1-193.

Pérez Hidalgo, N., Umaran, A., Mier Durante, M. P. \& Nieto Nafría, J. M., 2009. Aportaciones a la afidofauna íbero-balear (Hemiptera, Aphididae) a partir de las fotografías, y de sus metadatos, depositadas en el "Banco taxonómico faunístico digital de los invertebrados ibéricos" (B.T.F.D.I.I.). Graellsia, 65(2): 171-181. http:// dx.doi.org/10.3989/graellsia.2009.v65.i2.149

Quednau, F. W., 1990. Two new genera and three new species of drepanosiphine aphids from the Nearctic and Neotropical Regions (Homoptera: Aphididae). The Canadian Entomologist, 122: 907-919. http://dx.doi. org/10.4039/Ent122907-9

Quednau, F. W. \& Remaudière, G., 1994. Le genre sud-américain Neuquenaphis Blanchard (E.E.), description de deux nouvelles espèces et définition de nouvelles sous-familles d'Aphididae (Homoptera). Bulletin de la Société Entomologique de France, 99(4): 365-384.

Quirós, D. I. \& Emmen, D. A., 2006. Diversidad biológica de los áfidos (Hemiptera: Aphididae) de Panamá. Tecnociencia, 8(2): 68-75.

Quiroz, A., Fuentes-Contreras, E., Ramírez, C. C., Russell, G. B. \& Niemeyer, H. M., 1999. Host-plant chemicals and distribution of Neuquenaphis on Nothofagus. Journal of Chemical Ecology, 25(5): 1043-1054. http://dx. doi.org/10.1023/A:1020825707922
Quiroz, C., 1980. Estudios del efecto del complejo áfidovirus en el rendimiento del trigo en el valle centro norte de Chile. Agricultura Técnica (Chile), 40(1): 1-6.

Quiroz, C., Zúñiga, E, \& Ramírez, A., 1986. Vegetación cordillerana costera y andina como fuente de áfidos (Hom.: Aphididae) que afectan la producción de trigo. Agricultura Técnica (Chile), 46(3): 271-276.

Ramírez, C. C., 2002. Registro de algunas especies de áfidos (Hemiptera: Aphididae) en el Parque Nacional Laguna San Rafael. Boletín del Museo Nacional de Historia Natural (Chile), 51: 117-121.

Remaudière, G., 1994. Revue et clé des espèces sud-américaines d'Aphidina et description d'un Aphis nouveau [Homoptera Aphididae]. Revue Française d'Entomologie (Nouvelle Série), 16(3): 109-119.

Remaudière, G., Starý, P. \& Gerding, M., 1993. Sitobion fragariae (Walker) y Metopolophium festucae cerealium Stroyan (Homoptera: Aphididae), dos nuevos áfidos de los cereales, en Chile. Agricultura Técnica (Chile), 53(1): 91-92.

Richards, W. R., 1968. Neuquenaphis papillata, a new nothofagus-infesting aphid from Chile, with a review of related species from South America (Homoptera: Aphididae). The Canadian Entomologist, 100(11): 11991207. http://dx.doi.org/10.4039/Ent1001199-11

Ripa, R., Larral, P. \& Rojas, S., 2008a. Pulgón de la espírea, pulgón verde de los cítricos, pulgón verde del manzano, Spiraea aphid, Aphis spiraecola Patch. In: R. Ripa \& P. Larral (eds.). Manejo de plagas en paltos y citricos. Instituto de Investigaciones Agropecuarias. La Cruz: 123-129.

Ripa, R., Larral, P. \& Rojas, S., 2008b. Pulgón negro de los cítricos, Black citrus aphid, Toxoptera aurantii (Boy. de Fons.). In: R. Ripa \& P. Larral (eds.). Manejo de plagas en paltos y cítricos. Instituto de Investigaciones Agropecuarias. La Cruz: 129-132.

Ripa, R., Larral, P. \& Rojas, S., 2008c. Pulgón del melón, pulgón del algodonero, Melon aphid, Cotton aphid, Aphis gossypii Glover. In: R. Ripa \& P. Larral (eds.). Manejo de plagas en paltos y citricos. Instituto de Investigaciones Agropecuarias. La Cruz: 132-134.

Ripa, R., Luppichini, P. \& Larral, P., 2010. Plagas del nogal: nuevos desafios. http://www.chilenut.cl/ exponut2010/assets/docs/presentaciones/Ripa.pdf [Consulta julio 2014].

Rojas, S., 1966. Identificación de insectos entomófagos. Agricultura Técnica (Chile), 26(4): 173-175.

Rubio-Meléndez, M. E., Zamudio, F. \& Ramírez, C. C., 2011. Susceptibilidad de híbridos de Populus spp. al ataque de áfidos y royas en tres localidades de Chile. Bosque, 32: 127-132.

Servicio Agrícola y Ganadero, 2005a. Detecciones de plagas. Informativo Fitosanitario Forestal, 1(1): 1.

Servicio Agrícola y Ganadero, 2005b. Nueva distribución de plagas. Informativo Fitosanitario Forestal, 1(1): 1-2.

Servicio Agrícola y Ganadero, 2005c. Nuevos hospederos de plagas. Informativo Fitosanitario Forestal, 1(2): 1-2.

Servicio Agrícola y Ganadero, 2006a. Detecciones de plagas. Informativo Fitosanitario Forestal, 2(3): 1. 
Servicio Agrícola y Ganadero, 2006b. Nueva distribución de plagas. Informativo Fitosanitario Forestal, 2(3): 2.

Servicio Agrícola y Ganadero, 2010. Detecciones de plagas. Informativo Fitosanitario Forestal, 3(4): 2.

Smith, C. F. \& Cermeli, M., 1979. An annotated list of Aphididae (Homoptera) of the Caribbean Islands and South and Central America. North Carolina Agricultural Research Service Technical Bulletin, 299: $1-131$.

Starý, P., 1976. Two new Pseudephedrus Starý, aphid parasites (Hymenoptera: Aphidiidae) associated with Nothofagus in South America. With notes on the continental drift. Entomologica Scandinavica, 7: 24-30.

Starý, P., 1993. The fate of released parasitoids (Hymenoptera: Braconidae, Aphidiinae) for biological control of aphids in Chile. Bulletin of Entomological Research, 83: 633-639. http://dx.doi.org/10.1017/S0007485300040062

Starý, P., 1994. Aphid Parasitoid Fauna (Hymenoptera, Aphidiidae) of the Southern Beech (Nothofagus) Forest. Studies on Neotropical Fauna and Environment, 29: 87-98. http://dx.doi.org/10.1080/01650529 409360921

Starý, P., 1995. The Aphidiidae of Chile (Hymenoptera. Ichneumonoidea, Aphidiidae). Deutsche Entomologische Zeitschrift (Neue Folge), 42(1): 113-138. http://dx. doi.org/10.1002/mmnd.19950420112

Starý, P., Rakhshani, E., Žikić, V., Kavallieratos, G., Lavandero, B. \& Tomanović, Ž., 2014. Altitudinal zonation of aphid parasitoids (Hymenoptera: Braconidae:Aphidiinae) in the Neotropical Region. Entomological News, 124(2): 86-97. http://dx.doi.org/10.3157/021.124.0203

Starý, P., Rodríguez, F., Gerding, M., Norambuena, M. \& Remaudière, G., 1994a. Distribución, frecuencia, rango de hospederos y parasitismo de dos nuevas especies de áfidos de cereales: Sitobion fragariae (Walker) y Metopolophium festucae cerealium Stroyan (Homoptera, Aphididae), en Chile. Agricultura Técnica (Chile), 54(1): 54-59.

Starý, P., Rodríguez, F. \& Remaudière, G., 1994b. Asociación planta-áfidos-parasitoide (Hom., Aphidoidea;
Hym., Aphidiidae), en la zona central de Chile. Agricultura Técnica (Chile), 54(1): 46-53.

Suzuki, H., 1981. Hospederos alternativos de los parasitoides de los áfidos del trigo, en la V Región, Chile. Agricultura Técnica (Chile), 41(3): 165-167.

Tropicos.org. Missouri Botanical Garden, 2016. http:// www.tropicos.org [Consulta enero 2016].

van der Goot, P., 1912. Über einige wahrscheinlich neue Blattlausarten aus der Sammlung des Naturhistorischen Museums in Hamburg. Mitteilungen aus dem Naturhistorischen Museums in Hamburg, 29: 273-284.

Vargas, R. R., 1981. Hospederos secundarios de los áfidos del trigo. Agricultura Técnica (Chile), 41(4): 269-271.

Vargas, R. R. \& Suzuki, S. H., 1984. Evaluación de la acción de microhimenópteros parasitoides sobre los áfidos del trigo en una localidad de la $\mathrm{V}$ región de Chile. Agricultura Técnica (Chile), 44(1): 9-13.

Zerené, M., Caglevic, M. \& Ramírez, I., 1988. Un nuevo áfido de los cereales detectado en Chile. Agricultura Técnica (Chile), 48(1): 60-61.

Zúñiga, E., 1967a. Lista preliminar de áfidos que atacan cultivos en Chile, sus huéspedes y enemigos naturales. Agricultura Técnica (Chile), 27(4): 165-177.

Zúñiga, E., 1967b. Los pulgones del duraznero en Chile central. Agricultura Técnica (Chile), 27(1): 32-39.

Zúñiga, E., 1967c. Cuatro áfidos nuevos para Chile. Agricultura Técnica (Chile), 27(2): 87-91.

Zúñiga, E., 1968. Huéspedes para Chile del áfido Myzus persicae (Sulzer) (Homoptera: Aphididae). Revista Chilena de Entomología, 6: 145-146.

Zúñiga, E. \& Aguilera, A., 1989. Presencia del pulgón manchado Therioaphis trifolii (Monell) (Homoptera: Aphididae) en Chile; características y generalidades sobre su control. Agricultura Técnica (Chile), 49(2): 164-168.

Zúñiga, E. \& Suzuki, H., 1976. Ecological and economic problems created by aphids in Latin America. Outlook on Agriculture, 8(6): 311-319. http://dx.doi.org/10.1177/0030 72707600800602 